Florida International University FIU Digital Commons

6-12-2015

\title{
Multi-Objective Analysis and Optimization of Integrated Cooling in Micro-Electronics With Hot Spots
}

Sohail R. Reddy

Florida International University, sredd001@fiu.edu

DOI: $10.25148 /$ etd.FIDC000094

Follow this and additional works at: https:// digitalcommons.fiu.edu/etd

Part of the Aerodynamics and Fluid Mechanics Commons, Heat Transfer, Combustion Commons, and the Systems Engineering and Multidisciplinary Design Optimization Commons

\section{Recommended Citation}

Reddy, Sohail R., "Multi-Objective Analysis and Optimization of Integrated Cooling in Micro-Electronics With Hot Spots" (2015). FIU Electronic Theses and Dissertations. 2205.

https://digitalcommons.fiu.edu/etd/2205 


\title{
FLORIDA INTERNATIONAL UNIVERSITY
}

\author{
Miami, Florida
}

\section{MULTI-OBJECTIVE ANALYSIS AND OPTIMIZATION OF INTEGRATED COOLING IN MICRO-ELECTRONICS WITH HOT SPOTS}

\author{
A thesis submitted in partial fulfillment of the \\ requirements for the degree of \\ MASTER OF SCIENCE \\ in \\ MECHANICAL ENGINEERING \\ by \\ Sohail R. Reddy
}

2015 
To: Interim Dean Ranu Jung

College of Engineering and Computing

This thesis, written by Sohail R. Reddy, and entitled Multi-Objective Analysis and Optimization of Integrated Cooling in Micro-Electronics with Hot Spots, having been approved in respect to style and intellectual content, is referred to you for judgment.

We have read this thesis and recommend that it be approved.

Arvind Agarwal

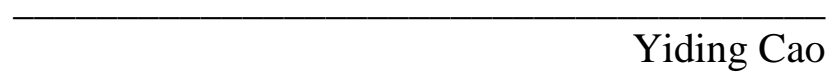

Bilal El-Zahab

Sakhrat Khizroev

George S. Dulikravich, Major Professor

Date of Defense: June 12, 2015

The thesis of Sohail R. Reddy is approved.

Interim Dean Ranu Jung

College of Engineering and Computing

Dean Lakshmi N. Reddi University Graduate School

Florida International University, 2015 


\section{DEDICATION}

I dedicate this thesis to my family for their constant support and sacrifice, without them this work would not have been possible. 


\section{ACKNOWLEDGMENTS}

First and foremost, I would like to thank Professor George Dulikravich, my major professor and my advisor, for always inspiring me and motivating me to reach to and beyond my potential. Thank you for fostering my passion for research and providing the opportunity to work on several interesting projects.

I would like to thank my committee members, Prof. Arvind Agarwal, Prof. Yiding Cao, Prof. Bilal El-Zahab and Prof. Sakhrat Khizroev for their fruitful discussions, encouragement and helpful comments.

I gratefully acknowledge the FIU Instructional and Research Computing Center for providing HPC resources to perform computations for this project. I thank Dr. Mengxing Cheng for his help with using FIU’s HPC resources.

I also thank Prof. Carlo Poloni, founder and president of ESTECO, for providing modeFRONTIER optimization software free of charge for this project.

I thank my friends and fellow labmates in MAIDROC: Cesar Pacheco, Rajesh Jha, Anthony Abrahao and Abas Abdoli for always making my time in the lab productive and interesting.

I am also deeply grateful for financial support provided by DARPA ICECool project grant HR0011-14-002 via GaTech grant RE314-G1, DOE/NETL grant DEFE002314 and AFOSR grant FA9550-12-0440. 


\title{
ABSTRACT OF THE THESIS \\ MULTI-OBJECTIVE ANALYSIS AND OPTIMIZATION OF INTEGRATED COOLING OF MICRO-ELECTRONICS WITH HOT SPOTS
}

by

\author{
Sohail R. Reddy \\ Florida International University, 2015 \\ Miami, Florida \\ Professor George S. Dulikravich, Major Professor
}

With computing power from electronic chips on a constant rise, innovative methods are needed for efficient thermal management. Arrays of micro pin-fins act not only as heat sinks, but also allow for the electrical interconnection between stacked layers of integrated circuits. This work performs a multi-objective optimization of three shapes of pin-fins to maximize the efficiency of this cooling system. An inverse design approach that allows for the design of cooling configurations without prior knowledge of thermal mapping was proposed and validated. The optimization study showed that pin-fin configurations are capable of containing heat flux levels of next generation electronic chips without compromising the structural integrity. The inverse approach identified configurations capable of cooling heat fluxes beyond those of next generation chips. Analysis of thin film heat spreaders made of diamond and graphene nanoplatelets showed that further reduction in temperature and thermal stresses, and increase in temperature uniformity are possible. 


\section{TABLE OF CONTENTS}

CHAPTER

PAGE

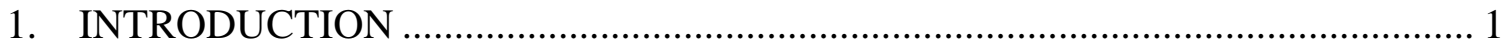

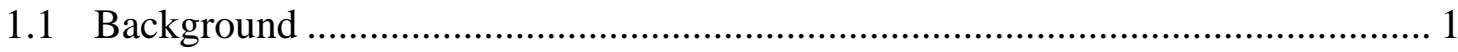

1.2 Techniques for Thermal Management ………………………………………... 3

1.3 Chosen Techniques for Thermal Management ………………………………....... 6

1.4 Objectives of the Research .................................................................................... 10

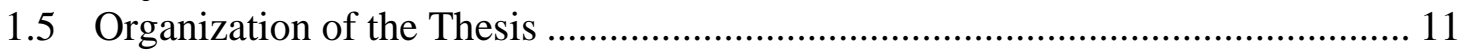

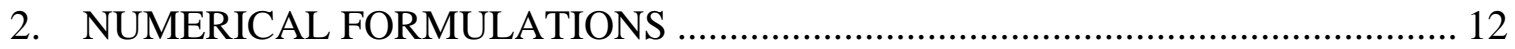

2.1 Conjugate 3D Heat Transfer Analysis............................................................... 12

2.1.1 Fluid Flow Model................................................................................... 12

2.1.2 Solid Region Model ............................................................................... 14

2.2 Stress-Deformation Analysis......................................................................... 14

2.3 Numerical Solution Procedure ..................................................................... 16

2.3.1 Domain Discretization............................................................................ 16

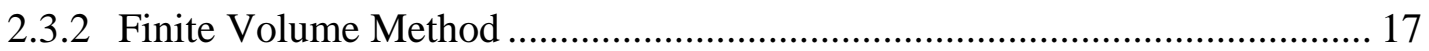

2.3.3 Velocity-Pressure Coupling ……………………........................................ 18

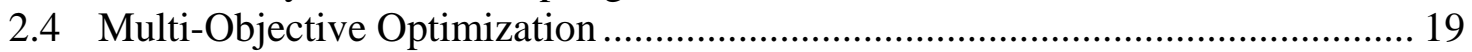

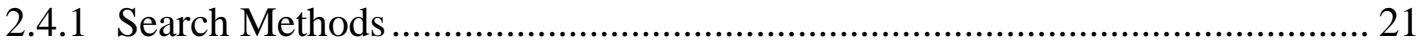

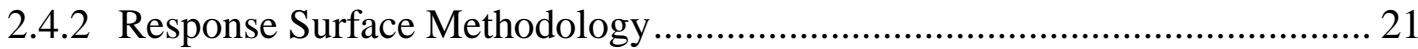

2.5 Inverse Design Concept of Electronic Cooling Configurations............................. 23

3. MULTI-OBJECTIVE OPTIMIZATION OF MICRO-PINS FINS............................. 25

3.1 Geometry Definition of Micro Pin-Fins .............................................................. 25

3.2 Multi-Objective Optimization for Uniform Heat Flux....................................... 27

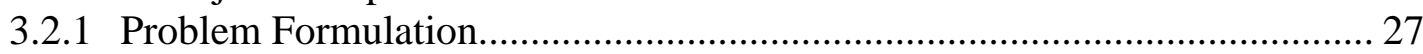

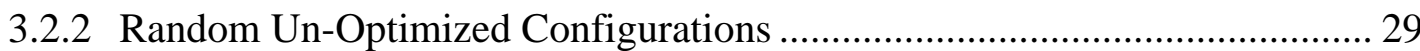

3.2.3 Optimized Configurations ............................................................................... 30

3.2.4 Stress-Deformation Analysis of Optimized Configurations ............................ 35

3.3 Multi-Objective Optimization for Electronic Chips with a Hot Spot ................... 37

3.3.1 Problem Formulation..................................................................................... 38

3.3.2 Random Un-Optimized Configurations ………………................................... 38

3.3.3 Optimized Configurations ............................................................................. 40

3.3.4 Stress-Deformation Analysis of Optimized Configurations ……………….... 44

4. INVERSE DESIGN AND PERFORMANCE MAP CONCEPT ............................... 47 
4.1 Problem Formulation................................................................................... 47

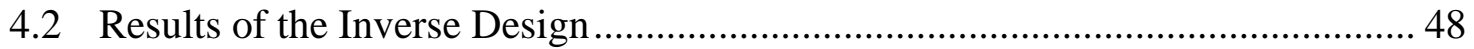

5. CONJUGATE ANALYSIS OF PIN-FIN ARRAYS WITH HEAT SPREADERS.. 53

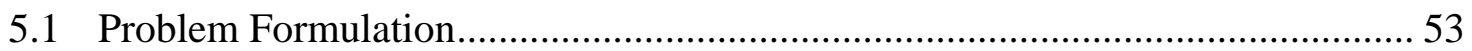

5.2 Conjugate Analysis: Diamond Thin Films ………………………………......... 54

5.3 Conjugate Analysis: Graphene Nano-Platelets Thin Films................................. 59

5.4 Conjugate Analysis: Segmented Thin Films ........................................................ 64

5.5 Conjugate Analysis of Thin Film: Summary …………….................................... 67

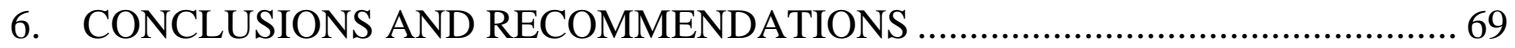

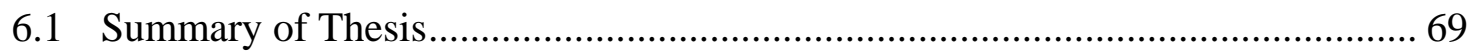

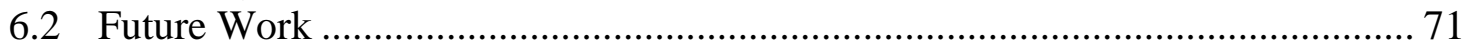

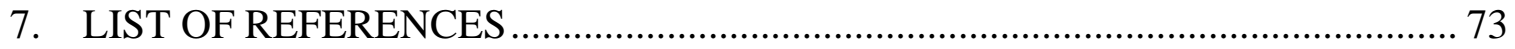




\section{LIST OF TABLES}

TABLE

PAGE

Table 1. Formulations of Radial Basis Functions utilized for response surfaces used in this study

Table 2. Range for design variables defining inlet conditions and the pin-fin configuration

Table 3. Parameters and objective function values for the three Pareto-optimized pin-fin

geometries

Table 4. Objective function values for non-optimized and Pareto-optimized arrays of micro pin-fins for each of the three pin-fin geometries.

Table 5. Parameters and objective function values for the three Pareto-optimized pin-fin geometries

Table 6. Objective function values for non-optimized and Pareto-optimized arrays of micro pin-fins for each of the three pin-fin geometries

Table 7. Range of design parameters and increment sizes.

Table 8. Parameters and boundary conditions for Pareto optimized configurations 50

Table 9. Geometric parameters and boundary conditions used for each analysis

Table 10. Maximum temperature $\left({ }^{\circ} \mathrm{C}\right)$ for the three pin-fin configurations with the top surface of the top wall coated with diamond heat spreader for various film thicknesses. 68

Table 11. Maximum temperature $\left({ }^{\circ} \mathrm{C}\right)$ for the three pin-fin configurations with the top surface of the top wall coated with graphene nano-platelets heat spreader for various film thicknesses. 


\section{LIST OF FIGURES}

FIGURE

PAGE

Figure 1. Maximum heat flux reported over the years by [2] ...................................2

Figure 2. Maximum heat flux loads managed by different cooling technologies [2].......3

Figure 3. Two-floor configuraiton having rectangular microchannels [4] ....................4

Figure 4. An array of micro pin-fins having circular cross sections housing Through

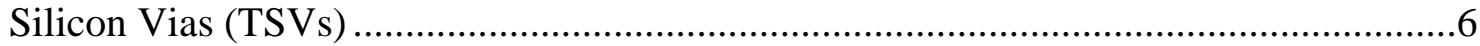

Figure 5. An array of micro pin-fins having: a) circular, b) airfoil, and c) convex cross sections. Only one half of the entire array is shown and analyzed because of

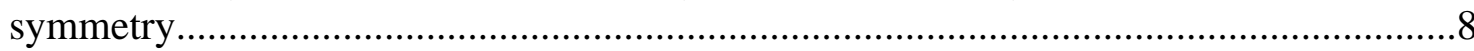

Figure 6. Typical hybrid computational grid used for the conjugate heat transfer analysis

Figure 7. SIMPLE algorithm flow chart ........................................................18

Figure 8. Typical convergence history of the conjugate heat transfer analysis

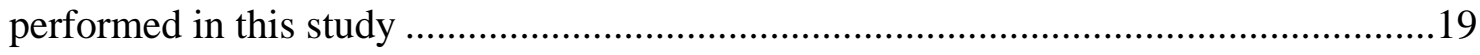

Figure 9. Workflow of different stages and software used .....................................20

Figure 10. Formulation of the inverse design problem ..........................................24

Figure 11. Dimension of the chip used in this study..........................................26

Figure 12. A micro-pin fin cooling configuration with pins having: a) circular, b) symmetric airfoil, and c) symmetric convex cross sections featuring a hot spot. ..........26

Figure 13. Temperature distribution for non-optimized configurations of pin fins having: a) circular, b) airfoil, and c) convex cross sections........................................29

Figure 14. Pressure field for non-optimized configurations of pin fins having: a) circular, b) symmetric airfoil, and c) symmetric convex cross sections. 
Figure 15. Objective function space for initial population and virtual Pareto designs for arrays of micro pin-fins having: a) circular, b) airfoil, and c) convex cross sections.

Figure 16. Temperature distribution for the optimized configurations of pin fins having: a) circular, b) airfoil, and c) convex cross sections.

Figure 17. Pressure field for optimized configurations of pin fins having: a) circular, b) symmetric airfoil, and c) symmetric convex cross sections.

Figure 18. Velocity magnitudes and flow vectors for the three Pareto optimized pin fin configurations

Figure 19. Displacement field due to hydrodynamic and thermal loads on Pareto optimized pin fin having: a) circular, b) airfoil and c) symmetric convex cross section36

Figure 20. Von-Mises stress distribution due to hydrodynamics and thermal loads on pin fins having: a) circular, b) airfoil and c) symmetric convex cross section

Figure 21. Temperature distribution for the non-optimized configurations of pin fins having: a) circular, b) airfoil, and c) convex cross sections.

Figure 22. Pressure field for non-optimized configurations of pin fins having: a) circular, b) symmetric airfoil, and c) symmetric convex cross sections.

Figure 23. Temperature field on the bottom surface for non-optimized configurations of pin fins having: a) circular, b) symmetric airfoil, and c) symmetric convex cross sections.

Figure 24. Objective function space for initial population and virtual Pareto designs for array of micro pin-fins having: a) circular, b) symmetric airfoil, c) symmetric convex cross sections, and d) superimposed Pareto frontiers for the three configurations showing that micro pin-fins with convex cross sections offer superior performance

Figure 25. Temperature distribution for the optimized configurations of pin fins having: a) circular, b) airfoil, and c) convex cross sections.

Figure 26. Temperature field on the bottom surface for optimized configurations of pin fins having: a) circular, b) symmetric airfoil, and c) symmetric convex cross sections. 
Figure 27. Displacement field due to hydrodynamic and thermal loads on Pareto optimized pin fin having: a) circular, b) airfoil and c) symmetric convex cross section45

Figure 28. Von-Mises stress distribution due to hydrodynamics and thermal loads on pin fins having: a) circular, b) airfoil and c) symmetric convex cross section

Figure 29. Three-dimensional Pareto frontiers for arrays of pin fins having: a) circular, b) airfoil and c) symmetric convex cross section

Figure 30. Temperature distribution for the optimized configurations of pin fins having: a) circular, b) airfoil, and c) convex cross sections

Figure 31. Temperature field on the bottom surface for optimized configurations of pin fins having: a) circular, b) symmetric airfoil, and c) symmetric convex cross sections

Figure 32. Pressure field for optimized configurations of pin fins having: a) circular, b) symmetric airfoil, and c) symmetric convex cross sections

Figure 33. Temperature distribution on the top surface of the top wall of the array of micro pin-fins with: a) circular, b) airfoil and c) convex cross sections and without heat spreader

Figure 34. Temperature distribution on the top surface of the top wall of the array of micro-pin fin array having circular cross section pin fins with: a) $5 \mu \mathrm{m}$, b) $10 \mu \mathrm{m}$ and c) $15 \mu \mathrm{m}$ diamond heat spreader.

Figure 35. Temperature variation along: a) sample line 1 and b) sample line 2 for the diamond coated top surface of the top wall of an array of micro pin-fins having circular cross sections.

Figure 36. Temperature distribution on the top surface of the top wall of the array of micro-pin fin array having airfoil cross section pin fins with: a) $5 \mu \mathrm{m}$, b) $10 \mu \mathrm{m}$ and c) $15 \mu \mathrm{m}$ diamond heat spreader.

Figure 37. Temperature variation along: a) sample line 1 and b) sample line 2 for the diamond coated top surface of the top wall of an array of micro pin-fins having airfoil cross sections.

Figure 38. Temperature distribution on the top surface of the top wall of the array of micro-pin fin array having convex cross section pin fins with: a) $5 \mu \mathrm{m}$, b) $10 \mu \mathrm{m}$ and c) $15 \mu \mathrm{m}$ diamond heat spreader. 
Figure 39. Temperature variation along: a) sample line 1 and b) sample line 2 for the diamond coated top surface of the top wall of an array of micro pin-fins having convex cross sections.

Figure 40. Temperature distribution on the top surface of the top wall of the array of micro-pin fin array having circular cross section pin fins with: a) $5 \mu \mathrm{m}$, b) $10 \mu \mathrm{m}$ and c) $15 \mu \mathrm{m}$ graphene nano-platelets heat spreader.

Figure 41. Temperature variation along: a) sample line 1 and b) sample line 2 for the graphene nano-platelets coated top surface of the top wall of an array of micro pinfins having circular cross sections.

Figure 42. Temperature distribution on the top surface of the top wall of the array of micro-pin fin array having airfoil cross section pin fins with: a) $5 \mu \mathrm{m}$, b) $10 \mu \mathrm{m}$ and c) $15 \mu \mathrm{m}$ graphene nano-platelets heat spreader.

Figure 43. Temperature variation along: a) sample line 1 and b) sample line 2 for the graphene nano-platelets coated top surface of the top wall of an array of micro pinfins having airfoil cross sections.

Figure 44. Temperature distribution on the top surface of the top wall of the array of micro-pin fin array having convex cross section pin fins with: a) $5 \mu \mathrm{m}$, b) $10 \mu \mathrm{m}$ and c) $15 \mu \mathrm{m}$ graphene nano-platelets heat spreader.

Figure 45. Temperature variation along: a) sample line 1 and b) sample line 2 for the graphene nano-platelets coated top surface of the top wall of an array of micro pinfins having convex cross sections.

Figure 46. View of the top surface of the electronic chip showing partial coating using thin films

Figure 47. Temperature distribution on the top surface of the top wall of the array of micro-pin fin array with: a) circular, b) airfoil and c) convex cross section and with segmented diamond heat spreader

Figure 48. Temperature distribution on the top surface of the top wall of the array of micro-pin-fins with: a) circular, b) airfoil, and c) convex cross section and with segmented graphene nano-platelets heat spreader

Figure 49. Temperature variation along: a) sample line 1 and b) sample line 2 for array of micro pin-fins having airfoil cross section and fully and partially coated with diamond and graphene nano-platelets. 


\section{CHAPTER 1}

\section{INTRODUCTION}

This section consists of a brief review and challenges in cooling and thermal management of electronics. Various techniques of thermal managements are presented and the proposed technique is discussed. Previous work done on the thermal management techniques is identified and objectives of this work are stated.

\subsection{Background}

Integrated circuits (ICs) of today operate at a heat flux level of approximately 100 $\mathrm{W} / \mathrm{cm}^{2}$, where the level of heat flux is assumed to be directly proportional to the operating (clock) speed of the IC. These types of ICs are usually found in laptops, TVs, cell phones and defense equipment. With the number of transistors per square inch of IC doubling every two years according to Moore's Law, the performance output, and consequently the heat fluxes, are on a constant rise (Figure 1). The heat flux levels in the next generation chips are predicted to reach $500 \mathrm{~W} / \mathrm{cm}^{2}$ at the background and in excess of $1000 \mathrm{~W} / \mathrm{cm}^{2}$ at the hot spots [1]. This dramatic increase in heat flux levels directly increases the IC temperatures outside of the electronic chip's optimum operating condition, thereby leading to reduced performance. The higher temperatures also increase thermal stresses, which over time can lead to fatigue and structural failure. 


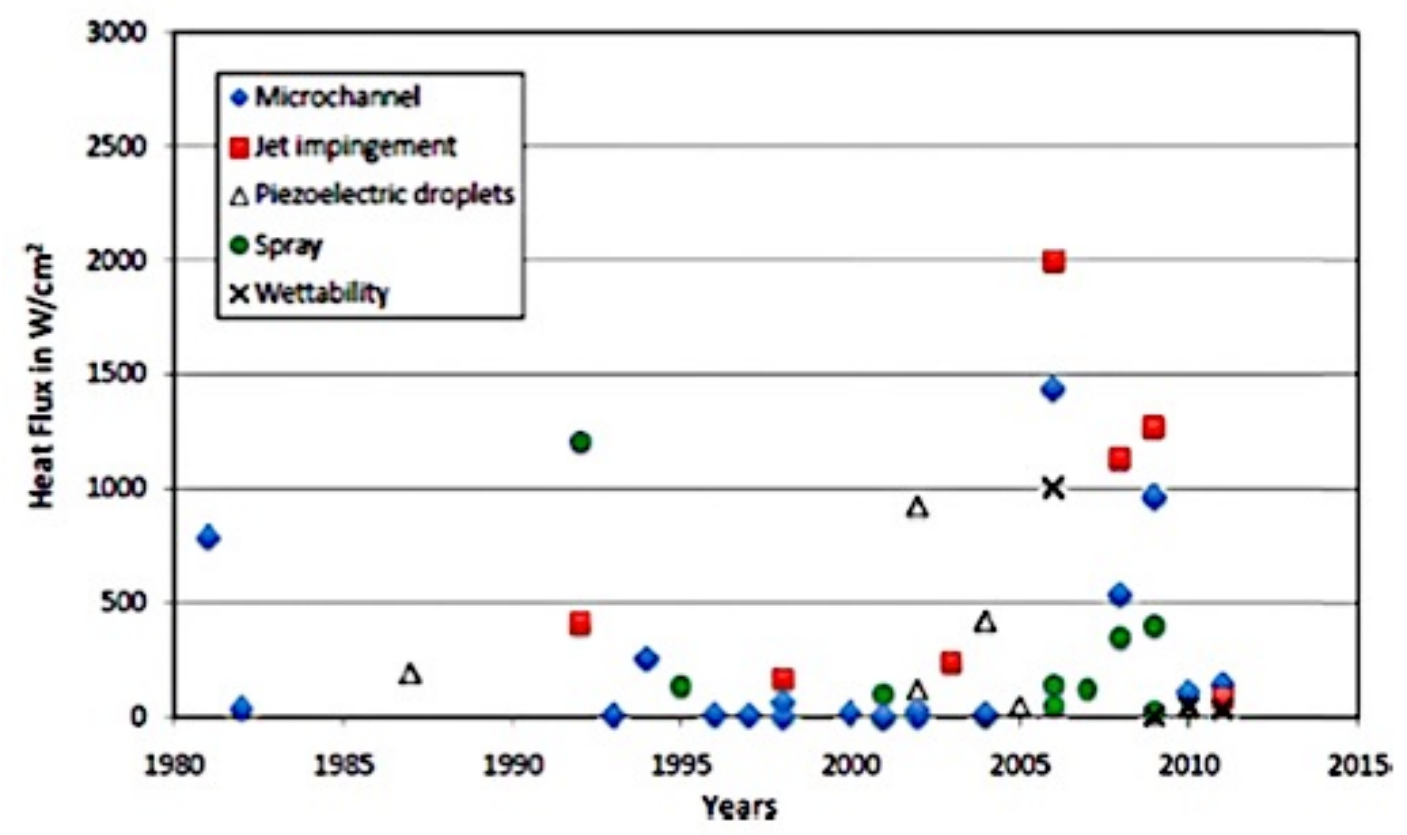

Figure 1. Maximum heat flux reported over the years by [2]

Over the last three decades various methods have been investigated for their ability to cope with such heat fluxes. Figure 2 shows the various methods applied for cooling high heat flux and the maximum reported heat flux that each cooling method is capable of handling. Figure 1 and Figure 2 show that many attempts and efforts have been made to investigate configurations capable of containing such high heat fluxes.

A traditional method of cooling such high heat flux configurations is through forced convection which requires a fluid, usually water or a refrigerant, to be pumped through the IC to remove the thermal energy. Although this dramatically increases the heat transfer performance of the system, it usually comes at a cost. The geometry and the configuration of the cooling system directly determine the input power required to pump the fluid (pumping power). This conflict of design objectives can be overcome by performing a multi-objective optimization. 


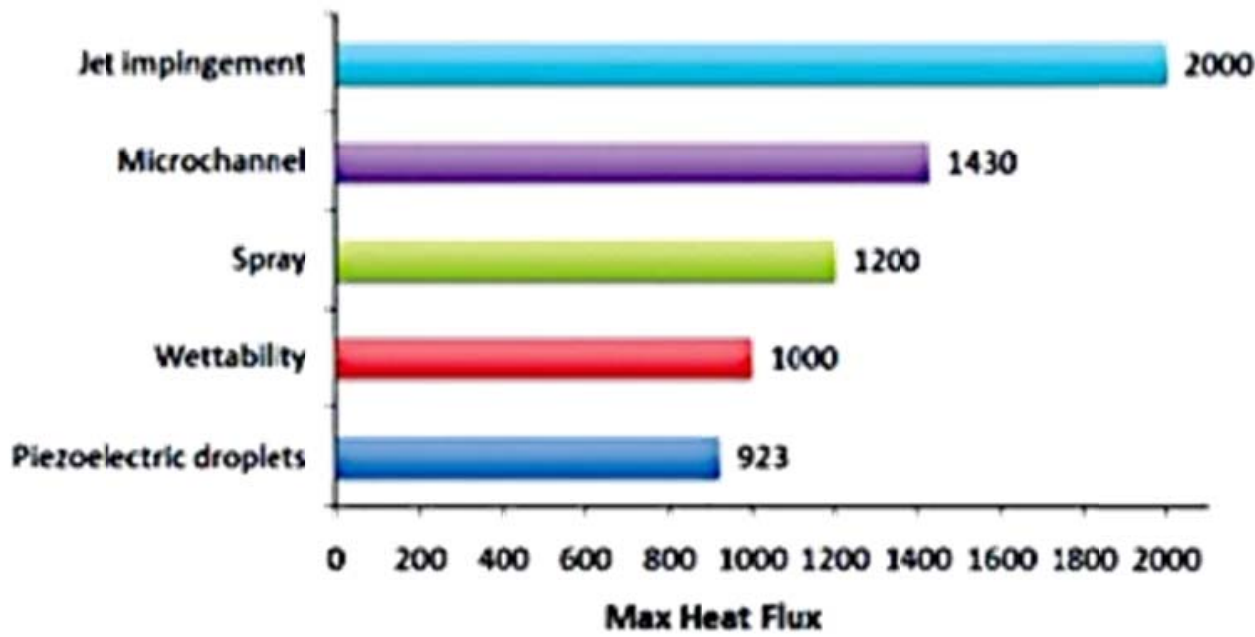

Figure 2. Maximum heat flux loads managed by different cooling technologies [2]

Multi-objective optimization is a method for multiple criteria decision-making where a problem with multiple objectives is being solved. Unlike single-objective optimization, the multi-objective optimization does not result in a single solution. Instead, multiple Pareto solutions (best trade-off solutions) are obtained. This multi-objective optimization technique can be applied to the design of electronic cooling configuration where the objective is to increase thermal efficiency, while reducing the pumping power.

\subsection{Techniques for Thermal Management}

One of the oldest and most common methods for heat removal is through the use of microchannel heat sinks. This method utilizes forced convection as the major mode of heat transfer where a working fluid, either water or coolant, is passed through micro- 
passages to remove the thermal energy. The cross sections of these micro-passages can vary significantly to attain the required performance and efficiency.

Wu and Mudawar [3] performed three-dimensional conjugate heat transfer analysis on microchannel heat sinks with rectangular cross sections. The heat flux considered in their work was $90 \mathrm{~W} / \mathrm{cm}^{2}$ and was applied uniformly at the top surface. They observed the temperature increase in the streamwise direction was linear and that increasing inlet velocity increases fluid entry length, which in turn enhances heat transfer.

Abdoli et al. [4] performed thermo-fluid-stress analysis on two floor microchannels having rectangular cross section shown in Figure 3. The heat flux of $1000 \mathrm{~W} / \mathrm{cm}^{2}$ was applied at the background and $2000 \mathrm{~W} / \mathrm{cm}^{2}$ at the hot spot was applied in their analysis. These heat flux levels closely mimic those of next generation electronics. Their research showed that their configuration of microchannels was able to keep the maximum temperature and maximum Von-Mises stress within acceptable range.

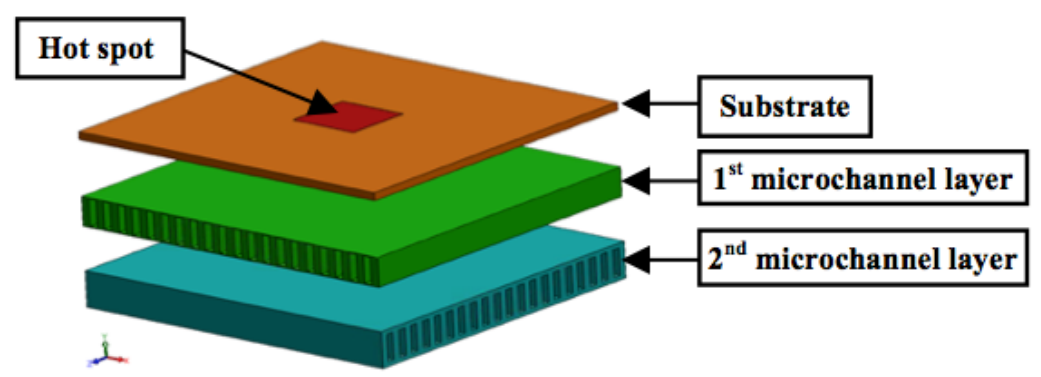

Figure 3. Two-floor configuraiton having rectangular microchannels [4]

Abdoli and Dulikravich performed multi-objective optimization of multi-floor counterflow [5] and throughflow [6] microchannel heat exchangers. The heat flux 
considered for their work was $1000 \mathrm{~W} / \mathrm{cm}^{2}$ uniformly applied at the top surface. Their work consisted of four objectives:

1. Maximize total amount of heat removed

2. Minimize coolant pressure drop

3. Minimize maximum temperature

4. Minimize temperature non-uniformity

In recent years, micro-jet cooling has been getting more attention in the thermal management community. This involves directly spraying the hot surface with an array of liquid micro-jets to reduce temperatures in the region. This method can be further divided into jet impingement and synthetic jets. Jet impingement requires an external source of fluid and some means for pumping, whereas synthetic jets are formed from the entrainment and expulsion of the fluid in which they are present. Micro-jet cooling is very attractive since micro-jets can be directed onto hot spots throughout the system. It not only decreases maximum temperature, but also temperature non-uniformity.

Fabbri and Dhir [7] optimized an array of micro-jets for cooling of high performance electronics using an experimental approach. Their work showed that a maximum heat flux of $310 \mathrm{~W} / \mathrm{cm}^{2}$ is attainable while keeping maximum temperature below a threshold.

Husain et al. [8] used numerical methods, coupled with surrogate models and a genetic algorithm to perform a multi-objective optimization. The two objective functions in their work were thermal resistance and pumping power. The optimum design was able to contain a heat flux of $100 \mathrm{~W} / \mathrm{cm}^{2}$ at a maximum temperature of $66^{\circ} \mathrm{C}$ and a pressure drop of about $24 \mathrm{kPa}$. 


\subsection{Chosen Techniques for Thermal Management}

Although micro-channel and micro-jet do not pose manufacturing difficulties, they do not allow for compact packaging of the IC. As electronic chips decrease in size, more compact configurations are needed. In today's semiconductor industry, this is overcome by using arrays of micro pin-fins. Micro pin-fin arrays, shown in Figure 4, not only act as heat sinks, carrying heat via conduction away from the heated surface, but also allow for electrical interconnection between stacked layers of chips. This is due to copper vias, referred to as Through-Silicon Vias (TSVs), housed inside of the silicon pin-fins.

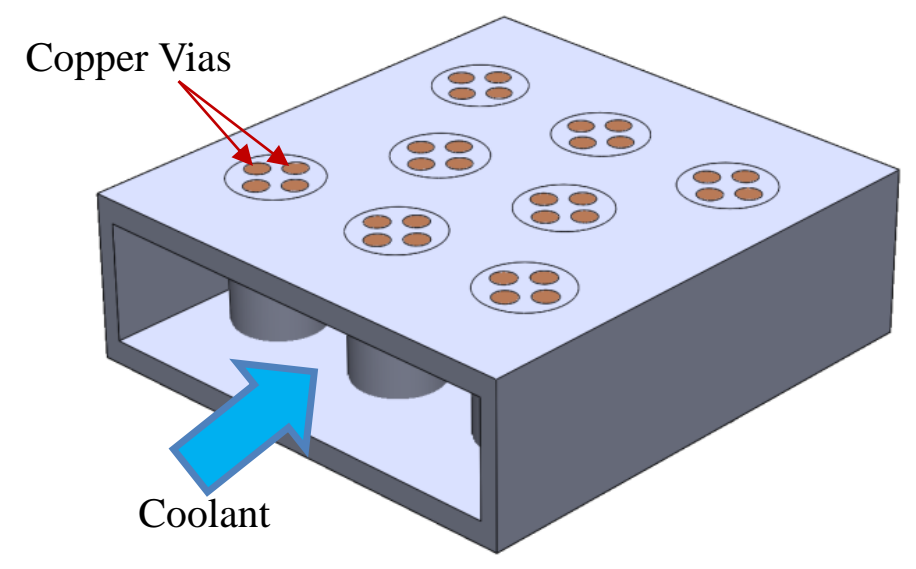

Figure 4. An array of micro pin-fins having circular cross sections housing Through Silicon Vias (TSVs)

Micro pin-fins have been studied in great detail by several researchers. Alfieri et al. [9] numerically studied the effects of size and distribution of the pin-fins on the thermal performance of 3D stacked circuits with integrated cooling. Their work considered a background heat flux of $50 \mathrm{~W} / \mathrm{cm}^{2}$ and $125 \mathrm{~W} / \mathrm{cm}^{2}$ at the hot spot. It was noticed that 
increasing the pin-fin diameter directly under the hot spot resulted in $20 \%$ lower temperatures.

In another work, Alfieri et al. [10] computationally modeled the vortex shedding in water-cooled array of micro pin-fins. Their research was focused on analyzing the friction, pressure, and drag coefficients, Strouhal number, pumping power, chip temperature and Nusslet number. Their work showed that even at low Reynolds Number, vortex shedding is present which is incorporated into the numerical models used in this work.

The more commonly implemented pin fin configurations feature a circular cross section. It was seen that circular cross section leads to flow separation and creates a recirculation region behind the pin fins. This separation reduces heat transfer and increases the temperatures in this region. Kosar and Peles [11] and Ndao et al. [12] experimentally investigated pin fins with four cross sections: circular, square, hydrofoil and elliptic. It was found that the circular and square pin-fins offered higher heat transfer coefficient due to the increased surface area.

Abdoli et al. [13] numerically investigated single floor and double floor, staggered arrays of micro pin-fins having circular, airfoil and convex cross sections (Figure 5). Their work showed that certain airfoil shapes can lead to lower temperatures than circular cross section having the same surface area. It was also shown that pin-fins with airfoil cross sections require significantly less pumping power to attain similar thermal performance. It should be mentioned that no optimization was performed and that further gains are possible should the configurations be optimized. 


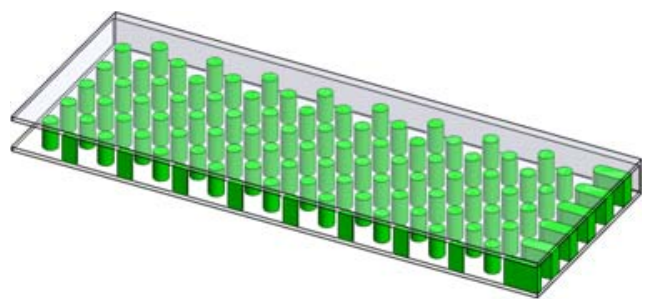

a)

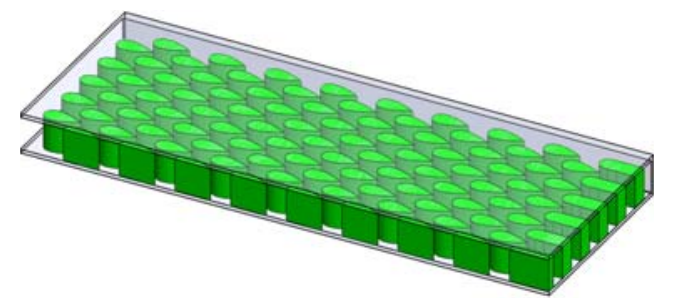

b)

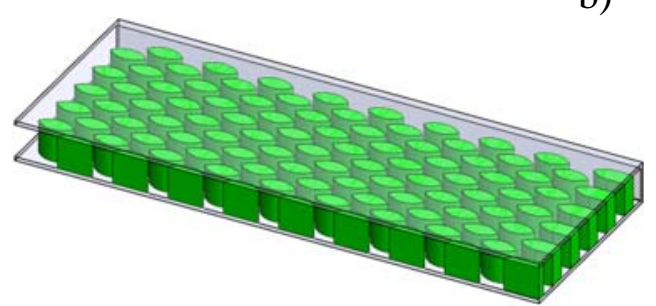

c)

Figure 5. An array of micro pin-fins having: a) circular, b) airfoil, and c) convex cross sections. Only one half of the entire array is shown and analyzed because of symmetry.

Tullius et al. [14] performed parametric optimization of six micro pin-fin cross sections: circular, square, triangle, ellipse, diamond and hexagon. Their work lead to important correlations between the parameters defining the configuration, Nusselt number and heat transfer coefficients. Their work does not provide absolute or Pareto optimum configurations, suggesting that only a parametric study was performed.

Although various cooling methods have been optimized [15, 16, 17], very little work has been done on optimizing micro pin-fin shapes. Even fewer efforts have been dedicated to optimizing various shapes under the same operating conditions for fair comparison. This work addresses the issue by optimizing various micro pin-fin configurations under the same thermal mapping.

A pattern can be discerned from the reported literature. The traditional method for designing cooling configurations requires that the heat flux be known prior to the design stage. This approach allows for the design of electronic cooling configuration for 
containing a specified heat flux. However, what happens when the heat flux levels change? Can a cooling configuration be developed without prior knowledge of operating heat flux levels? This can be formulated in a question form as follows.

Given

Maximum allowable temperature of the material,

Inlet cooling fluid temperature,

Total pressure loss (pumping power affordable), and

Overall thickness of the entire electronic component,

what is the maximum possible heat flux at the hot spot and background under which the temperature will not exceed the maximum allowable value?

This work formulates and validates this new inverse approach to design of cooling configurations that can be used without prior knowledge of heat flux operating levels. This inverse design problem is solved using a constrained multi-objective optimization algorithm.

Although next generation chips are expected to generate $1000 \mathrm{~W} / \mathrm{cm}^{2}$ at hot spots, certain military applications operate at much higher levels. Such high heat flux level cannot be contained using traditional methods and more sophisticated methods must be sought. One such method is the use of thin film heat spreaders to dissipate the heat away from hot spots, thereby reducing the maximum temperature at the hot spot.

Fukutani and Shakouri [18] previously investigated Si and SiGe superlattice thin film micro-coolers. They found that the hot spot temperature can be lowered by 10 to $30^{\circ} \mathrm{C}$ at a heat flux of $1000 \mathrm{~W} / \mathrm{cm}^{2}$. Their work also showed that an optimum film thickness exists that provides the highest cooling density at the hot spots. Wang et al. [19] employed 
embedded thin film thermo-electric cooler for isothermalization of individual chips. They showed this method could eliminate more than $90 \%$ of the temperature non-uniformity on the electronic chip.

Much like the work done by Singh et al. [20] and Labounty et al. [21], the work presented in this thesis investigates diamond thin films applied to micro pin-fin configurations, and their effects on maximum temperature and temperature uniformity. Smalc et al. [22] investigated the use of natural graphite as heat spreader that features a much higher thermal conductivity. Current work also investigates the use of graphene nano-platelets that offer much higher thermal conductivity at a lower material cost.

\subsection{Objectives of the Research}

The objective of this research is to perform a multi-objective optimization of forced convection cooling arrays of micro pin-fins having three different cross sections: circular, symmetric airfoil and symmetric convex. The thermal loads considered will be those of next generation electronic chips and will be kept constant for the three cross sections allowing for a fair comparison of their performances.

A new approach to design of such cooling configurations will also be developed and validated. This new approach will allow for the design of such a cooling configuration that will have the maximum possible performance subject to specified coolant inlet temperature and a specified maximum temperature. The objectives of the research are as follows: 
Perform 3D, steady-state, conjugate heat transfer analysis of each array of micro pin-fins

Perform multi-objective optimization for each of the three micro pin-fin geometries

Perform stress and deformation analysis of the optimized cooling configuration

Formulate and validate inverse design approach for each of the three micro pin-fin configurations

Perform 3D, steady-state, conjugate heat transfer analysis and investigate the effects of thin film heat spreaders

\subsection{Organization of the Thesis}

The remaining chapters are organized as follows:

$>$ Chapter II discusses governing equations, numerical procedures and problem formulation

$>$ Chapter III performs multi-objective optimization of arrays of pin-fins subjected to uniform and non-uniform heat fluxes

$>$ Chapter IV presents and validates the inverse design approach for the arrays of three micro pin-fin geometries

$>$ Chapter $\mathrm{V}$ performs conjugate heat transfer analysis on arrays of micro pin-fins with thin film heat spreaders

$>$ Chapter VI presents the discussion and recommendations for future work 


\section{CHAPTER 1I}

\section{NUMERICAL FORMULATIONS}

This chapter discusses the numerical formulation and governing equations used in each analysis. The formulation can be grouped into two distinct sections: 3D conjugate heat transfer analysis and stress-deformation analysis.

\subsection{Conjugate Heat Transfer}

The 3D conjugate heat transfer (CHT) is a coupled heat convection and heat conduction analysis in the entire solid-fluid configuration. This section is divided into the formulations for the fluid domain and solid domain. The system of partial differential equations governing the conjugate heat transfer was solved using the Finite Volume Method in ANSYS Fluent.

\subsubsection{Fluid Flow Model}

This work utilizes water as the heat removing fluid. The small length scale and low velocities lead to low Reynolds numbers. Although the Reynolds number might suggest laminar flow, Alfieri et al. [10] demonstrated it can still lead to vortex shedding. This turbulence is modeled using the standard $\kappa-\epsilon$ turbulence model. The Reynolds 
Averaged Navier-Stokes (RANS) equations were solved in the fluid domain. The RANS equations model turbulent flow by decomposing the instantaneous field variables into time-averaged and fluctuation of the field variables [23]. Collectively the RANS equations consist of mass, momentum and energy balance equations along with the equations governing turbulence quantities, $\kappa$ and $\varepsilon$.

For incompressible, steady-state and Newtonian fluids, the mass balance has the form

$$
\nabla \cdot \vec{V}=0
$$

where $\vec{V}$ is the velocity vector. The steady-state momentum balance takes the form

$$
\rho(\vec{V} \cdot \nabla) \vec{V}=-\nabla p+\nabla \cdot\left(\left(\mu+\mu_{T}\right)\left[\nabla \vec{V}+(\nabla \vec{V})^{T}\right]\right)
$$

Here $\mu$ and $\mu_{T}$ are the dynamic and turbulent viscosity coefficients of the fluid, respectively. The steady-state energy balance equation, neglecting viscous dissipation and heat sources, takes the form

$$
\rho C_{P}(\vec{V} \cdot \nabla) T=(\vec{V} \cdot \nabla) p+\nabla \cdot(k \nabla T)
$$

where $\rho, C_{P}$ and $k$ are the fluid density, specific heat at constant pressure and thermal conductivity, respectively.

The standard $\kappa-\epsilon$ turbulence model defines the turbulent viscosity as

$$
\mu_{T}=C_{\mu} \frac{\kappa_{T}}{\epsilon_{T}}
$$

where $\kappa_{T}$ and $\epsilon_{T}$ are the turbulence kinetic energy (TKE) and turbulent dissipation rate (TDR) respectively. The TKE and TDR for incompressible flows are defined as

$$
\begin{gathered}
\frac{\partial \kappa_{T}}{\partial t}+\nabla \cdot\left(\kappa_{T} \vec{V}-\frac{\mu_{T}}{\sigma_{K}} \nabla \kappa_{T}\right)=P-\epsilon_{T} \\
\frac{\partial \epsilon_{T}}{\partial t}+\nabla \cdot\left(\epsilon_{T} \vec{V}-\frac{\mu_{T}}{\sigma_{\epsilon}} \nabla \epsilon_{T}\right)=\frac{\epsilon_{T}}{\kappa_{T}}\left(C_{1} P-C_{2} \epsilon_{T}\right)
\end{gathered}
$$

where $P$ represents the production of turbulent kinetic energy defined as 


$$
P=\frac{\mu_{T}}{2}\left|\nabla \vec{V}+(\nabla \vec{V})^{T}\right|^{2}
$$

The constants in the scalar transport equations take the following values [24]:

$$
C_{1}=1.44 \quad C_{2}=1.92 \quad C_{\mu}=0.09 \quad \sigma_{\kappa}=1.0 \quad \sigma_{\epsilon}=1.3
$$

\subsubsection{Solid Region Model}

The governing equations for the solid region were solved only for the micro pin-fins and the chip casing. This region was defined as made of silicon. This model requires explicit specification of zero velocity in this region and that the material properties of the solid region be used in the governing equations. With these restrictions placed on the solid domain, the energy equation for the fluid domain reduces in the solid domain to the steady-state heat conduction equation and takes the form

$$
\nabla \cdot(k \nabla T)=0
$$

where $T$ is the absolute temperature and $k$ is the thermal conductivity of the solid material.

\subsection{Stress-Deformation Analysis}

Stress-deformation analysis was performed on each of the three optimized micro pinfin geometries to study the effects of thermos-elastic and hydrodynamic loads on the structural integrity of the cooling configuration. The steady-state conservation of linear momentum for a homogeneous isotropic body [25] is given by 


$$
\rho \frac{\partial^{2} \vec{u}}{\partial t^{2}}-\nabla \cdot \sigma-\overrightarrow{f_{b}}=0
$$

where $\vec{u}$ is the solid displacement vector, $\sigma$ is the stress tensor and $f_{b}$ is the body force. For the steady-state analysis, the first term on the left-hand side is neglected. The body force term is also neglected in this study. The stress tensor for a linear elastic body is defined as

$$
\sigma=2 G \varepsilon+\lambda \operatorname{tr}(\varepsilon) \mathrm{I}
$$

where $\lambda$ and $G$ are the first and second (shear modulus) Lame's coefficients, $\varepsilon$ is the strain tensor and I is the identity tensor. The first and second Lame coefficients are given by

$$
\lambda=\frac{E v}{(1+v)(1-2 v)} \quad G=\frac{E}{2(1+v)}
$$

where $E$ is the modulus of elasticity and $v$ is the Poission's ratio. The strain tensor, $\varepsilon$ is defined as

$$
\varepsilon=\frac{1}{2}\left[\nabla \vec{u}+(\nabla \vec{u})^{T}\right]+\alpha \Delta T \mathrm{I} \quad \Delta T=T-T_{0}
$$

where $\alpha$ is the coefficient of thermal expansion and $T_{0}$ is the reference temperature. The partial differential equations were solved using the Finite Element Method in ANSYS Structural [26].

The Von-Mises stress criterion is used to determine structural integrity of the cooling configuration. The objective of the design was to keep the maximum Von-Mises stress below the yield strength of silicon. Von-Mises stress is defined as 


$$
\sigma_{V M}=\sqrt{\frac{3}{2} \sigma^{\prime}: \sigma^{\prime}} \quad \sigma^{\prime}=\sigma-\frac{\operatorname{tr}(\sigma)}{3} \mathrm{I}
$$

where $\sigma^{\prime}$ is the deviatoric stress tensor.

\subsection{Solution Procedure}

This section discusses the solution procedure for performing the conjugate heat transfer analysis. It is sub-divided into three segments: Domain Discretization, Finite Volume Method, and Velocity-Pressure Coupling.

\subsubsection{Domain Discretization}

Domain discretization consists of decomposing the solution domain into smaller elements over which the governing equations are solved. For spatial discretization, the solution domain is decomposed into smaller control volumes. Because ANSYS Fluent is a cell-centered finite volume solver, the dependent variables are stored at the centroid of each cell. The computational grid in this work was of the hybrid kind with structured grid and unstructured grid throughout the domain. To fully capture the boundary layer phenomenon, five layers of clustered structured hexahedral grid cells were placed on each fluid-solid interface. The minimum allowable length scale of the cells was limited to

one micron to satisfy continuum so that the RANS equations can be solved for the fluid domain. The remainder of the solution domain was discretized using tetrahedral cells. 
The domain discretization was performed in ANSYS Meshing. Figure 6 shows a typical hybrid grid used in each analysis.
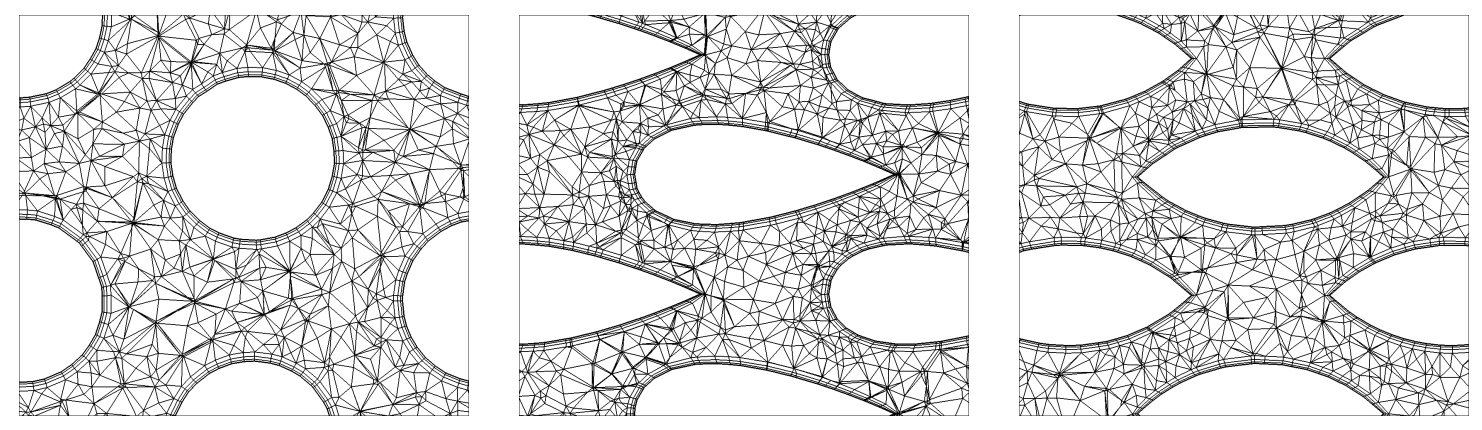

Figure 6. Typical hybrid computational grid used for the conjugate heat transfer analysis

\subsubsection{Finite Volume Method}

Having discretized the solution domains into control volumes, a method is still needed to solve the governing partial differential equations. This work utilizes the Finite Volume Method to solve the discretized governing equations over each of the control volumes. This method requires that the governing equations be integrated over each control volume and that the volume integral be converted to surface integral. The dependent variables are then evaluated as fluxes entering and leaving the control volume. This method is conservative since the flux entering the control volume is equal to the flux leaving the control volume. 


\subsubsection{Velocity-Pressure Coupling}

Having discretized the system of equation suitable for Finite Volume Method, a method for coupling the velocity and pressure is needed. This work utilized the SIMPLE [27] (Semi-Implicit Method for Pressure Linked Equations) algorithm to achieve this objective. The SIMPLE algorithm uses velocity-pressure relationship to enforce mass conservation and to obtain the pressure field. This pressure field does not satisfy the momentum conservation and must be corrected. The basis of the SIMPLE algorithm is this pressure correction that is iterated until both continuity and momentum conservation are satisfied. Figure 7 shows the flowcharts of the SIMPLE algorithm.

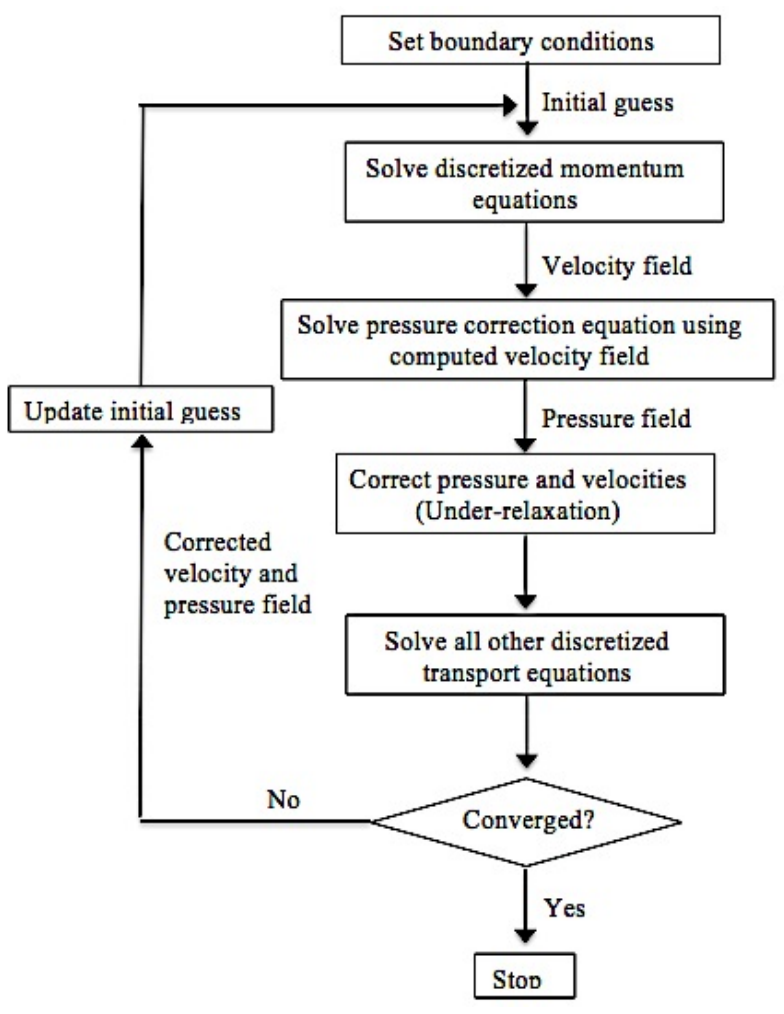

Figure 7. SIMPLE algorithm flow chart 
Figure 8 shows the typical convergence history of the 3D conjugate heat transfer analysis carried out in this work. It demonstrates that the conjugate heat transfer analysis has fully converged, thus producing high fidelity values of the objective functions.

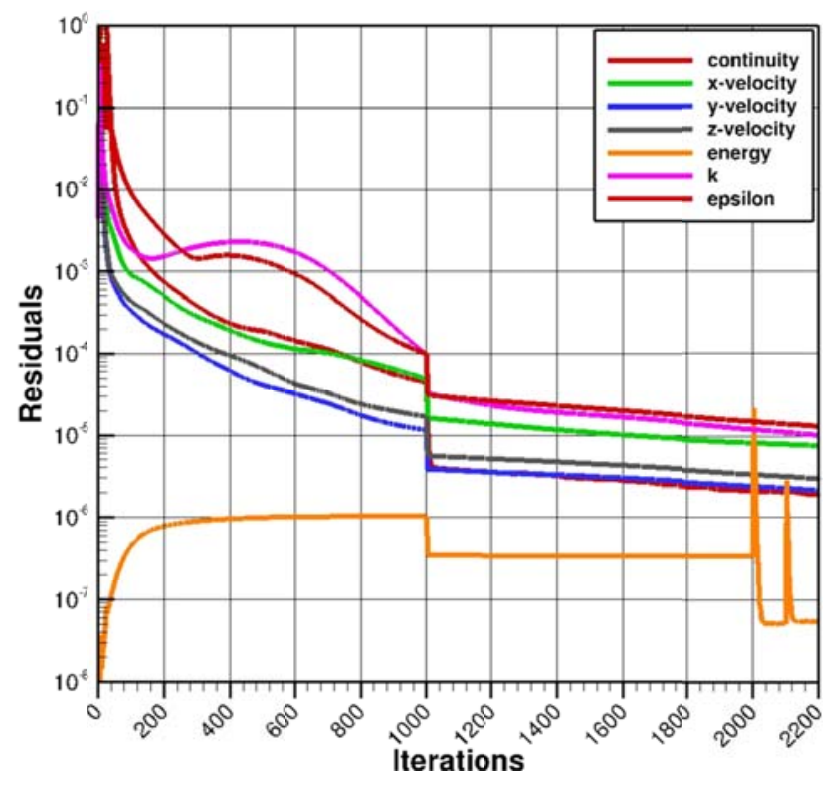

Figure 8. Typical convergence history of the conjugate heat transfer analysis performed in this study

\subsection{Multi-Objective Optimization}

The ultimate goal of this work is to perform multi-objective optimization of the three micro pin-fin configurations. Having discussed the solution procedure for the conjugate heat transfer and stress-deformation analysis, the optimization procedure is now presented. The optimization study consists of defining design variables that should be optimized to either maximize or minimize a certain function referred to as the objective function.. Figure 9 shows the typical optimization workflow used in this study. Each 
section of the optimization procedure is explained in further detail in the subsequent sections. All optimization was performed using commercial software modeFRONTIER [28].

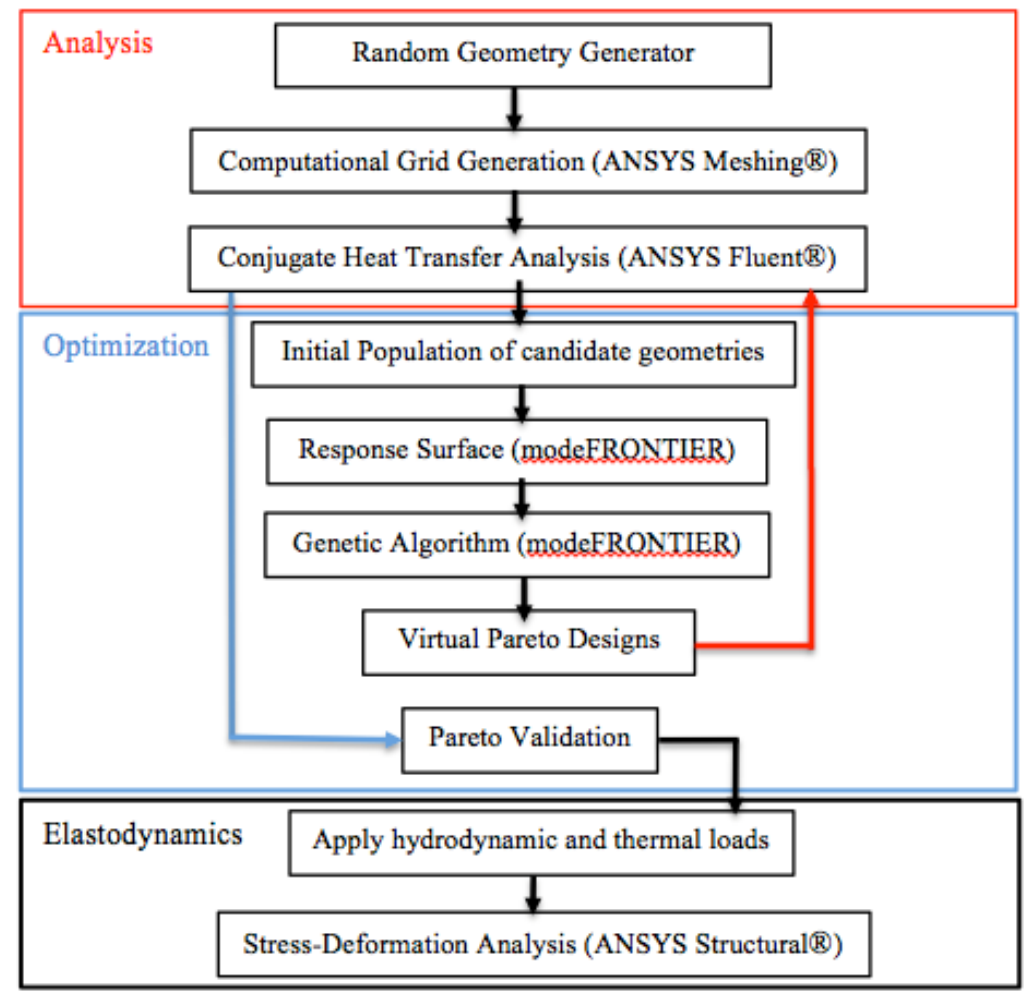

Figure 9. Workflow of different stages and software used

This methodology is used to optimize cooling configurations for a specified heat flux that exceeds the heat flux of next generation electronic chips. A modified version of the optimization set-up is presented in Section 2.6. 


\subsubsection{Search Methods}

There are two methods for performing optimization: deterministic and nondeterministic. Deterministic methods use gradient-based logic to arrive at the global extremum, but tend to converge to a local extremum. Some gradient-based methods include conjugate gradient method, BFGS and Sequential Quadratic Programming (SQP) [29]. Non-deterministic methods use some form of random sampling to avoid convergence to local extremas. Examples of non-deterministic methods include Particle Swarm, Genetic Algorithm and Predator Prey to name a few.

This work used the NSGA-II [30] (Non-Dominated Sorting Genetic Algorithm) to navigate through design space to search for optimum designs. The NSGA-II was selected as the search method because of its proven convergence. The NSGA-II's crowding distance operator and non-dominated sorting approach provide computationally efficient selection process and offer a wide spread of optimum designs.

\subsubsection{Response Surface Methodology}

Regardless of the search method used, a method for evaluating the objective function is needed. This can be achieved by directly coupling the analysis software to the NSGAII algorithm, but it would be computationally expensive since each conjugate heat transfer analysis requires seven hours to converge. For this reason, an efficient method of computing the objective function is needed. 
A response surface can be used to fit a set of high fidelity values of the objective function. An optimization algorithm can then very quickly read the interpolated values off the objective function of the response surface. The response surface in this work was constructed using Radial Basis Functions (RBF). The general formulation of RBF interpolation is given as

$$
y=\sum_{i=1}^{n} \omega_{i} \phi\left|x-x_{i}\right|
$$

where $n$ is the number of points used to construct the interpolation, $\omega$ are the weights obtained from solving the linear system of equations and $\phi$ is the radial distance between the points. The radial distance can vary depending on the RBF used. Table 1 shows the various formulations used in this work.

Table 1. Formulations of Radial Basis Functions utilized for response surfaces used in this study

\begin{tabular}{cc}
\hline Radial Basis Function & $\boldsymbol{\phi}\left(\left|\boldsymbol{x}_{\boldsymbol{i}}-\boldsymbol{x}_{\boldsymbol{j}}\right|\right)$ \\
\hline Hardy's Multiquadrics [31] & $\phi\left(\left|x_{i}-x_{j}\right|\right)=\sqrt{\left(x_{i}-x_{j}^{2}\right)+c^{2}}$ \\
Inverse Quadrics [32] & $\phi\left(\left|x_{i}-x_{j}\right|\right)=\left(\sqrt{\left(x_{i}-x_{j}^{2}\right)+c^{2}}\right)^{-1}$ \\
Gaussian [32] & $\phi\left(\left|x_{i}-x_{j}\right|\right)=e^{-c^{2}\left(x_{i}-x_{j}{ }^{2}\right)}$ \\
\hline
\end{tabular}

Here, $c$ is the shape factor that is determined using Leave-One-Out Cross Validation (LOOCV) [32]. All three radial basis functions were tested for each optimization run and the one with the smallest difference between the interpolated value and the computed value (ANSYS Fluent) was selected as the method of choice. It should be reported that one response surface is constructed for each objective function. Also, it should be noted 
that the input parameters to the response surface model are the design variables being optimized while the outputs are the interpolated objective function values.

It is known that the accuracy of any interpolation is heavily dependent on the method, number of interpolation points and the distribution of these points. Since the number of variables differs for each optimization done in this study, the number of points used to construct the response surface varies and is reported in the respective sections.

For accurate interpolation, an even distribution of points within the domain is needed. For this reason, the quasi-random sequence generation SOBOL's algorithm [33] was used to fill the design space.

\subsection{Inverse Design of Electronic Cooling Configurations}

The inverse approach presented in this work allows for the design of cooling configuration without having prior knowledge of operating heat flux level. The inverse design problem can be formulated in a question form:

"Given the fluid inlet conditions, what is the maximum allowable heat flux at the hot spot and background that can be imposed without exceeding the maximum allowable temperature?"

The inverse problem is solved using a constrained multi-objective optimization [34]. The previously presented methodology, genetic algorithm coupled with response surface, can also be applied to the inverse problem with minor adjustments to constraints and 
objective functions. Figure 10 shows the optimization set-up used to solve the inverse problem.

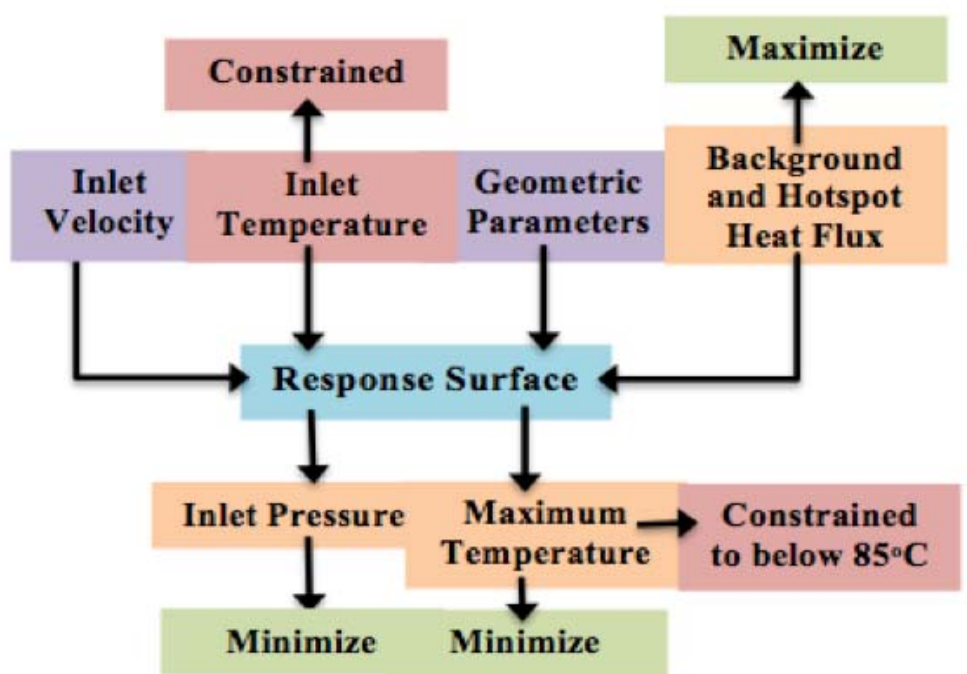

Figure 10. Formulation of the inverse design problem

This procedure was applied to each of the three micro pin-fin geometries for a fair comparison. The goal of the inverse problem is to identify a cooling pin-fin shape and scheme that is able to maximize the applied heat flux while minimizing the inlet pressure and keeping the maximum temperature from not exceeding the specified limit for the given material and inlet coolant temperature. 


\section{CHAPTER I1I}

\section{MULTI-OBJECTIVE OPTIMIZATION OF MICRO PIN-FINS}

This section discusses the results of the optimization study when the micro pin-fins are optimized for a specified heat flux. This approach is most commonly used in the industry. The section is divided into two sub-sections where the three cooling configurations are optimized for both uniform heat flux and non-uniform heat flux. The assumptions and optimization conditions for each study are reported in their respective sections.

\subsection{Geometry Definition of Micro Pin-Fins}

For any shape optimization study, the configuration must first be parameterized. This section presents the method for defining each of the three pin-fin shapes considered and the cooling configuration. All parameters presented in this section are also design variables that will later be optimized.

All electronic chips considered in this study have a footprint of $4 \times 3 \mathrm{~mm}$. Those electronic chips with hot spots have the hot spot dimension of $0.5 \times 0.5 \mathrm{~mm}$. Figure 11 shows one such chip featuring a hot spot and circular cross section pin-fins.

Each configuration features a channel outlet of the same thickness as the pin fins. This is done to suppress backflow and increase heat conduction at the outlet. The cooling 
configuration is also defined using chip height. This is done to allow for user's space constrained applications. The thickness of all sidewalls was kept constant at $30 \mu \mathrm{m}$.

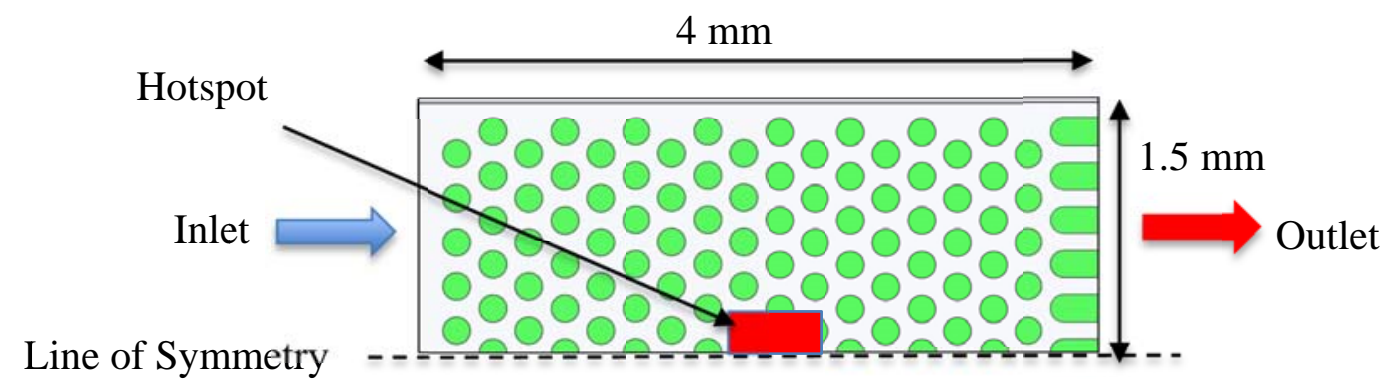

Figure 11. Dimension of the chip used in this study

The number of parameters defining the cross section of each pin-fin shape varies. The circular cross section pin-fins were defined using the pin-fin diameter. The symmetric airfoil and symmetric convex cross section pin-fins were defined using chord length and maximum thickness. The symmetric airfoil shape in this study was defined using a four series, symmetric NACA 00XX airfoils. Figure 12 shows the three shapes each with a hot spot. Only half of the chip is analyzed due to symmetry.

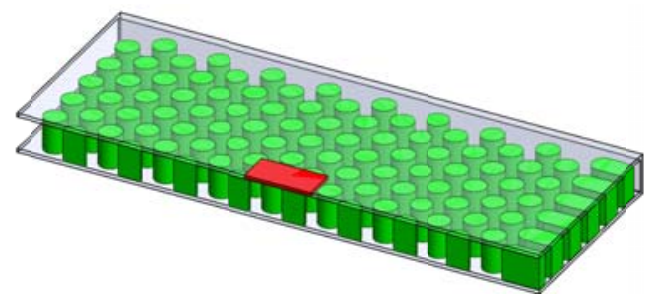

a)

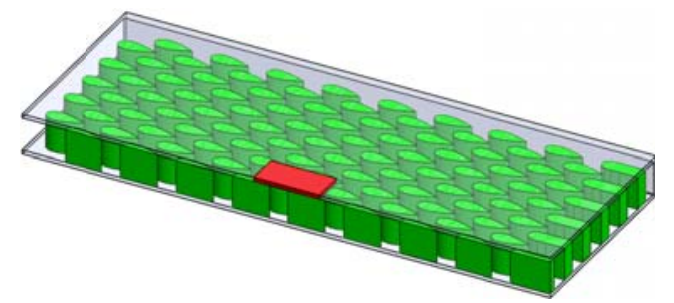

b)

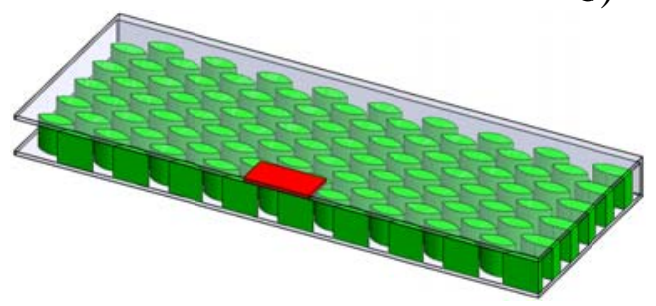

c)

Figure 12. A micro-pin fin cooling configuration with pins having: a) circular, b) symmetric airfoil, and c) symmetric convex cross sections featuring a hot spot. 
The spline equation defining this airfoil profile is given as

$$
y_{t}=5 t c\left[0.2969 \sqrt{\frac{x}{c}}-0.1260 \frac{x}{c}-0.3516\left(\frac{x}{c}\right)^{2}+0.2843\left(\frac{x}{c}\right)^{3}-0.1015\left(\frac{x}{c}\right)^{4}\right]
$$

where $c$ is the chord length, $x$ is the position along the chord from 0 to $c, t$ is the maximum relative thickness and $y_{t}$ is the half thickness at a given value of $x$.

\subsection{Multi-Objective Optimization for Uniform Heat Flux}

This section presents the optimization set up and results of the optimization study when applied to design of micro pin-fins under the influence of uniform heat flux. The section is divided into problem formulation, un-optimized configurations, optimized configurations and stress-deformation analysis of the optimized configurations.

\subsubsection{Problem Formulation}

For a fair comparison of the various micro pin-fin shapes, all three shapes (shown in Figure 5) were optimized under the same conditions. All three shapes were optimized for a uniform heat flux of $500 \mathrm{~W} / \mathrm{cm}^{2}$ on the top surface of the chip. A gauge pressure of $20 \mathrm{kPa}$ was applied at the outlet since cooling configurations are pressurized to avoid cavitation. The bottom surface along with all of the sidewalls was thermally insulated to 
assure that the fluid removes all of the heat. The inlet fluid temperature was kept constant at $30^{\circ} \mathrm{C}$.

The design variables for the optimization include both the geometric parameters as well as the inlet fluid velocity. For all optimization studies, a range must be specified for each design variable. This range is defined by the user to take into account constraints or manufacturing difficulties. The optimizer will then search within this range for the optimum design. Table 2 shows the range and the step size for each design variable.

Table 2. Range for design variables defining inlet conditions and the pin-fin configuration

\begin{tabular}{c|cc}
\hline Design Variable & Range & Step Size \\
\hline Inlet Velocity & $1-5 \mathrm{~m} / \mathrm{s}$ & $0.2 \mathrm{~m} / \mathrm{s}$ \\
Height & $100-250 \mu \mathrm{m}$ & $50 \mu \mathrm{m}$ \\
Diameter & $100-200 \mu \mathrm{m}$ & $10 \mu \mathrm{m}$ \\
Chord & $200-300 \mu \mathrm{m}$ & $10 \mu \mathrm{m}$ \\
Thickness & $80-160 \mu \mathrm{m}$ & $10 \mu \mathrm{m}$ \\
\hline
\end{tabular}

The two objectives of this study were:

1. Minimize maximum temperature

2. Minimize inlet pressure (pressure drop)

A constraint of maximum allowable temperature of $85^{\circ} \mathrm{C}$ was imposed in all optimization studies. Fully 3D conjugate heat transfer analysis was performed on 30 candidate designs having circular cross section pin-fins and 50 candidate designs having airfoil and convex cross section pin-fins. The response surfaces (one for each objective function) created with these data sets were coupled with the NSGA-II algorithm. 


\subsubsection{Random Un-Optimized Configurations}

To compare and test the performance of the optimization, one configuration for each pin-fin shape was randomly created using the SOBOL's algorithm. The results from the conjugate heat transfer analysis are presented in this section.

Figure 13 shows the temperature field for the random configurations of the three pinfin geometries. It can be seen that smaller heights result in much higher temperatures due to reduced heat transfer via convection.

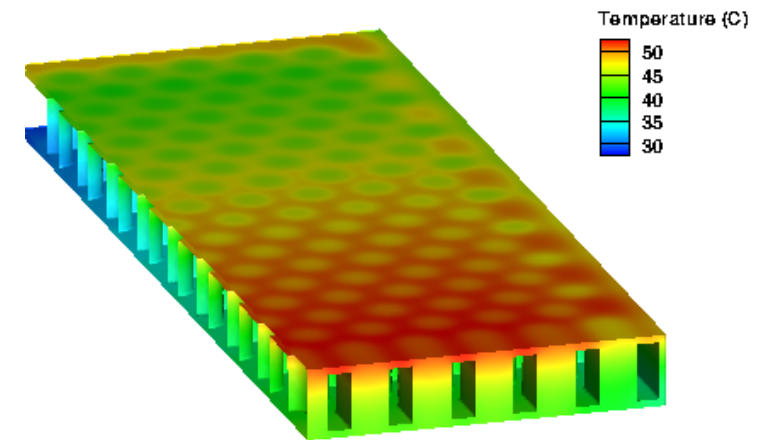

a)

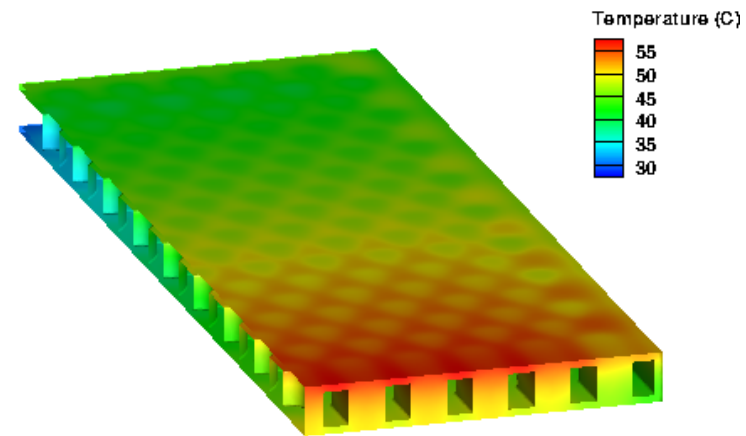

b)

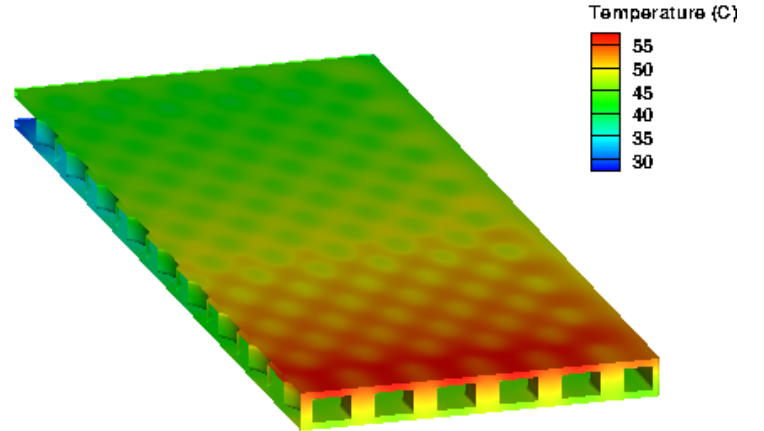

c)

Figure 13. Temperature distribution for non-optimized configurations of pin fins having: a) circular, b) airfoil, and c) convex cross sections.

Figure 14 shows the pressure field at mid-height of each configuration. 


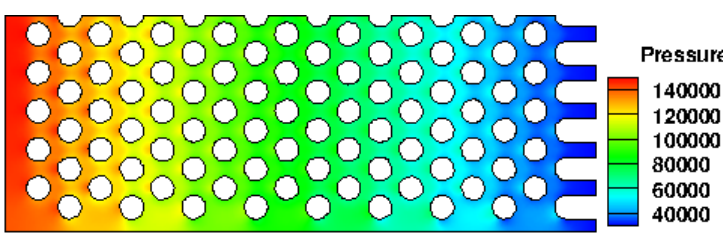

a)

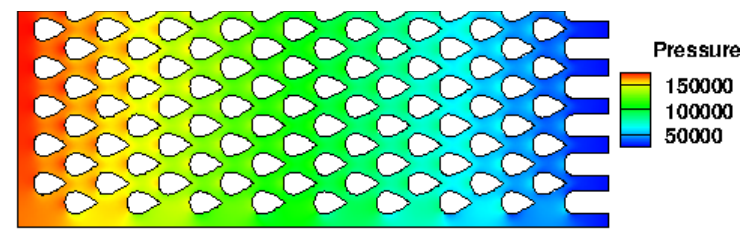

b)

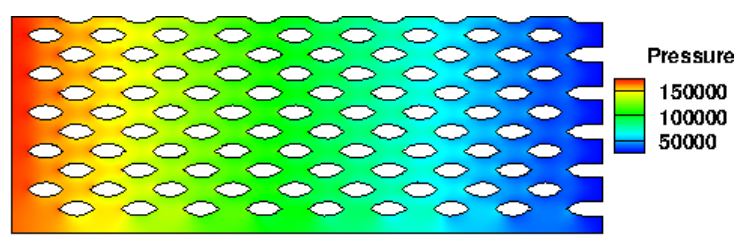

c)

Figure 14. Pressure field for non-optimized configurations of pin fins having: a) circular, b) symmetric airfoil, and c) symmetric convex cross sections.

\subsubsection{Optimized Configurations}

Figure 15 shows the Pareto fronts obtained using the NSGA-II algorithm. It shows the virtual Pareto designs (green) and the initial population (red) used to construct the response surface. Although the response surfaces were validated for certain designs, it is extremely difficult to achieve global accuracy. To investigate any local discrepancies in the response surface, four designs were selected from each of the three Pareto fronts, and were analyzed in ANSYS Fluent. It was found that the objective function values from the response surface deviated by less that 3\% from those obtained from the conjugate heat transfer analysis. 


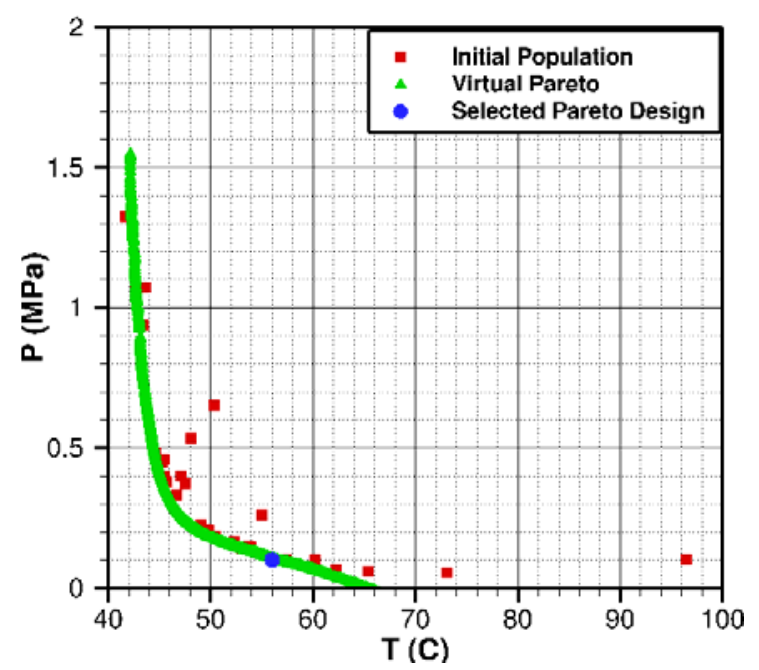

a)

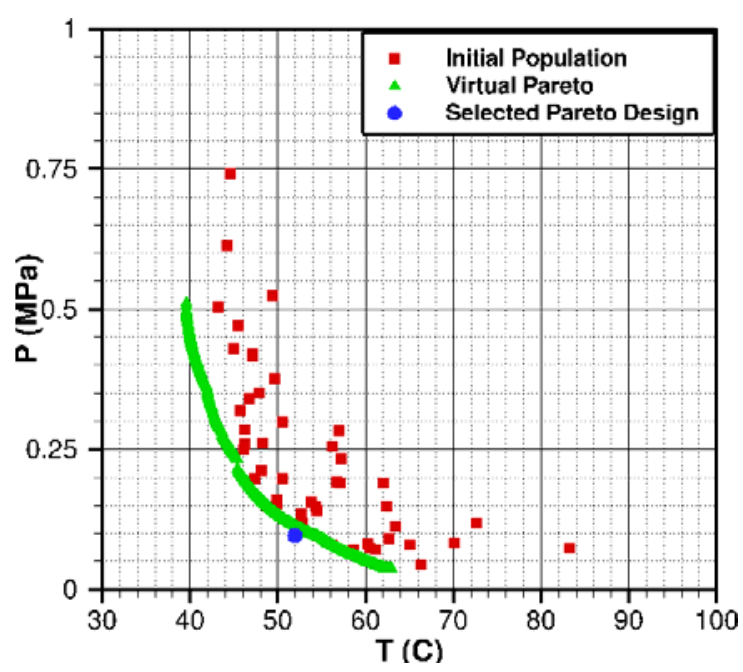

b)

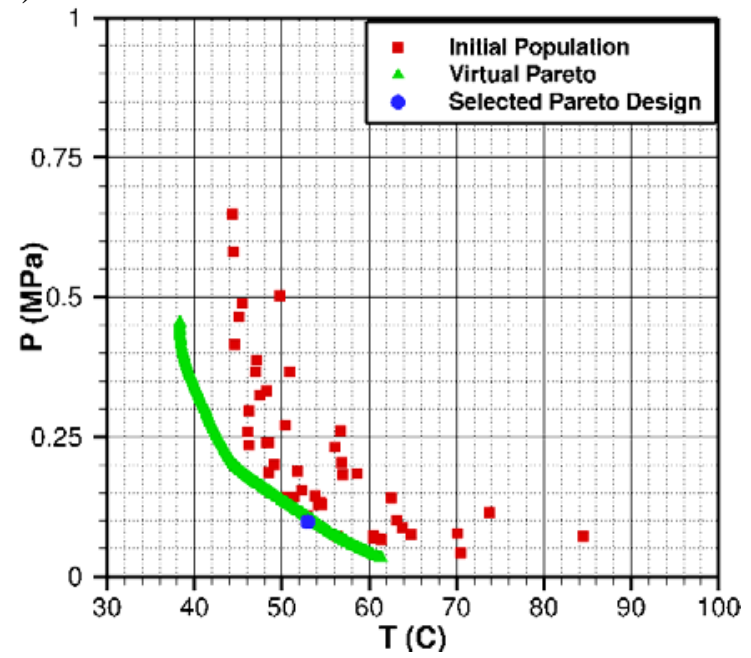

c)

Figure 15. Objective function space for initial population and virtual Pareto designs for arrays of micro pin-fins having: a) circular, b) airfoil, and c) convex cross sections.

Table 3 shows the geometric parameters defining each Pareto-optimized micro pin-fin configuration along with their respective objective function values. 
Table 3. Parameters and objective function values for the three Pareto-optimized pin-fin geometries

\begin{tabular}{c|ccc|}
\hline & Circular & Airfoil & Convex \\
\hline Diameter $(\boldsymbol{\mu m})$ & 120 & - & - \\
Chord Length $(\boldsymbol{\mu m})$ & - & 210 & 290 \\
Thickness $(\boldsymbol{\mu m})$ & - & 90 & 90 \\
Height $(\boldsymbol{\mu m})$ & 250 & 250 & 250 \\
Inlet Velocity $(\mathrm{m} / \mathrm{s})$ & 2.2 & 3 & 4.4 \\
\hline Maximum Temperature & 56 & 52 & 53 \\
$\left({ }^{\circ} \mathrm{C}\right)$ & 101.40 & 96.55 & 89.00 \\
\hline Inlet Pressure $(\mathrm{kPa})$ & & &
\end{tabular}

Figure 16 shows the temperature distribution through each of the three optimized pin-fin configurations. It can be seen from Figure 16 and Table 3 that the maximum temperature for the circular cross section pin-fins is higher than that of the pin-fins having airfoil and convex cross sections. This suggests that airfoil and convex cross section pin-fins are better able to transfer heat from the pin-fins to the moving fluid.

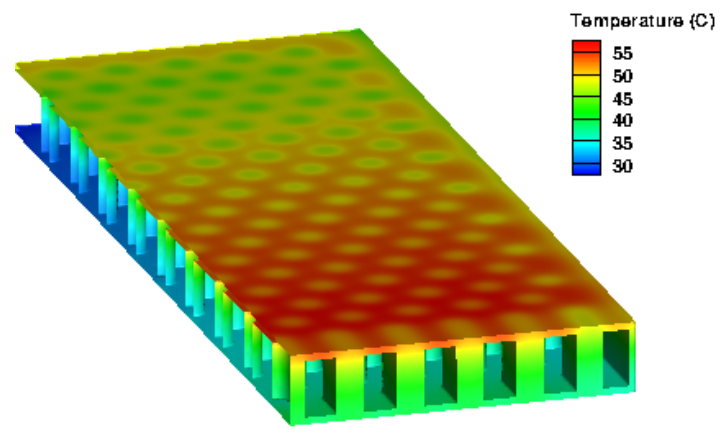

a)

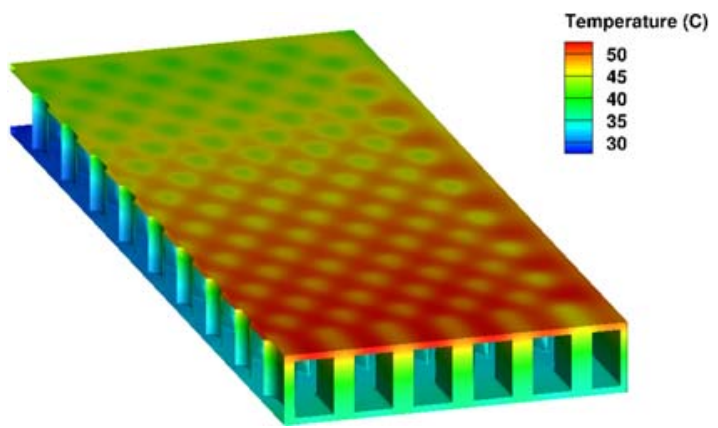

b) 


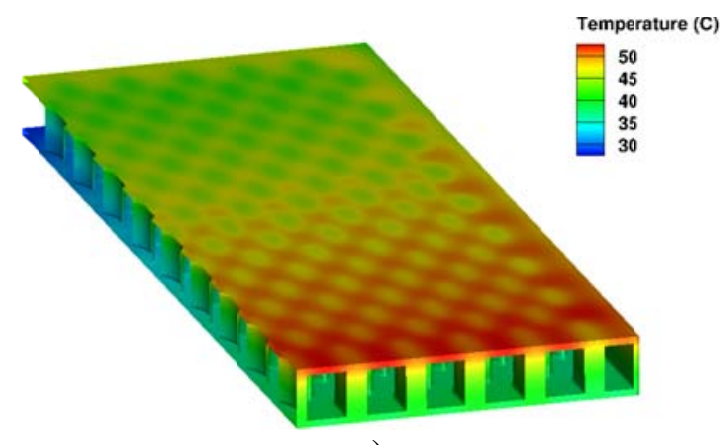

c)

Figure 16. Temperature distribution for the optimized configurations of pin fins having: a) circular, b) airfoil, and c) convex cross sections.

Figure 17 shows the pressure distribution on a slice placed at mid-height of each of the configurations. The high-pressure stagnation region can be seen at the leading edge of the circular and airfoil cross section designs. This high-pressure region has decreased in the convex cross section design. Figure 17 and Table 3 also shows that the circular micro-pin fin geometry requires the highest inlet pressure, followed by the airfoil and convex design.

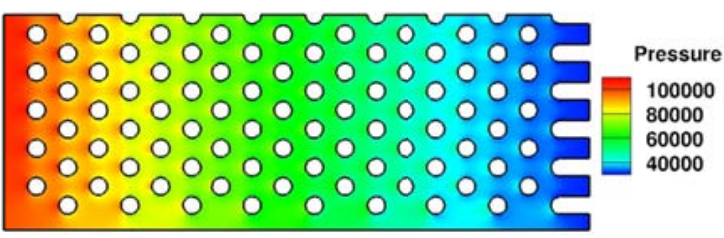

a)

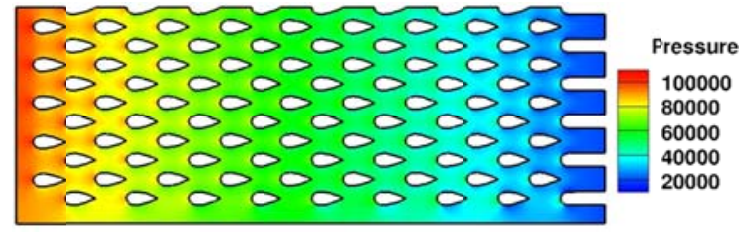

b)

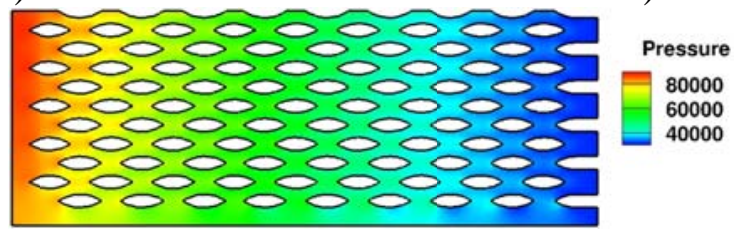

c)

Figure 17. Pressure field for optimized configurations of pin fins having: a) circular, b) symmetric airfoil, and c) symmetric convex cross sections. 
Figure 18 shows the velocity magnitude and flow vector fields for each of the three optimized configurations at mid height. It can be seen that even the optimized circular pin-fins experience severe flow separation. An enlarged view of the recirculation region is also visible. This phenomenon is greatly reduced in both the airfoil and convex configurations.
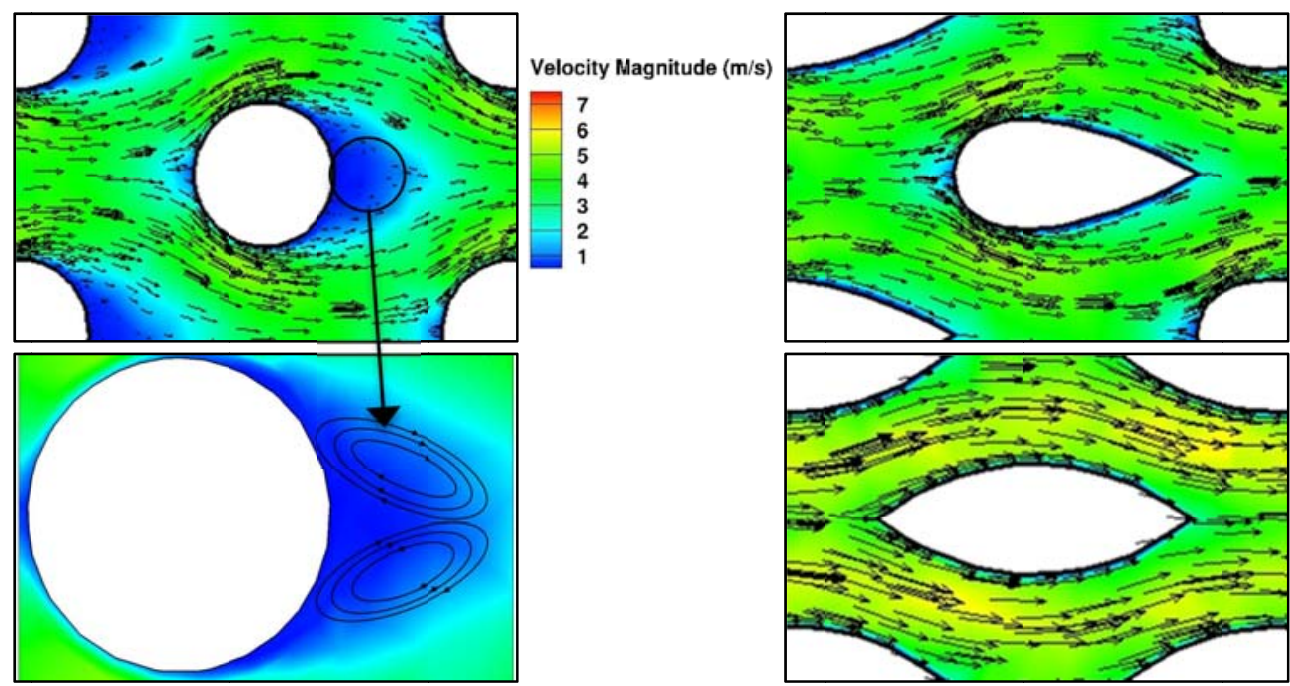

Velocity Magnitude $(\mathrm{m} / \mathrm{s})$ 8
-6

4
-2

Figure 18. Velocity magnitudes and flow vectors for the three Pareto optimized pin fin configurations

Table 4 shows the objective function values for the un-optimized and optimized configurations. It can be seen that both airfoil and convex cross section shapes resulted in lower temperature and pressure than circular cross section pin-fins. It should be mentioned that priority was given to lower pressure when selecting a Pareto design from the Pareto front, but another design can be selected that best satisfies the designer's needs. 
Table 4. Objective function values for non-optimized and Pareto optimized arrays of micro pin-fins for each of the three pin fin geometries.

\begin{tabular}{c|cccc}
\hline & $\begin{array}{c}\mathbf{T} \\
\left.\mathbf{}^{\mathbf{O}} \mathbf{C}\right)\end{array}$ & $\begin{array}{c}\Delta \mathbf{\Delta T} \text { from } \\
\text { un-optimized } \\
\text { configuration }\end{array}$ & $\mathbf{P ~ ( k P a )}$ & $\begin{array}{c}\Delta \mathbf{\Delta} \% \text { from } \\
\text { un-optimized } \\
\text { configuration }\end{array}$ \\
\hline $\begin{array}{c}\text { Un-Optimized } \\
\text { Circular }\end{array}$ & 54 & - & 146.18 & - \\
$\begin{array}{c}\text { Optimized } \\
\text { Circular }\end{array}$ & 56 & -4 & 101.4 & 30 \\
$\begin{array}{c}\text { Un-Optimized } \\
\text { Airfoil }\end{array}$ & 57 & - & 188.36 & - \\
$\begin{array}{c}\text { Optimized } \\
\text { Airfoil }\end{array}$ & 52 & 9 & 96.55 & 49 \\
$\begin{array}{c}\text { Un-Optimized } \\
\text { Convex }\end{array}$ & 56 & - & 180.47 & - \\
Optimized & 53 & 6 & 89.0 & 51 \\
\hline Convex & & & & \\
\hline
\end{tabular}

\subsubsection{Stress-Deformation Analysis of Optimized Configurations}

Depending on the sensitivity of the optimum designs, even the slightest deviation from the optimum configuration can lead to significant reduction in performance. The geometric deviations, for example, can be due to manufacturing defects, thermal expansion or hydrodynamic loads. These deformations can give rise to stresses high enough to cause structural failure. The displacement fields and stress fields were analyzed for each Pareto-optimized configuration where both thermal and hydrodynamic loads were incorporated into the model. All external sides of the chip were fixed so as to investigate the stresses and deformation on the pin-fins.

Figure 19 shows the displacement field for the three pin-fin configurations. It can be seen that even with the thermal and hydrodynamic loads, the maximum displacement is 
still on the nano-level. This suggests that geometric deviations, and therefore deviation from the optimum configuration, are miniscule.

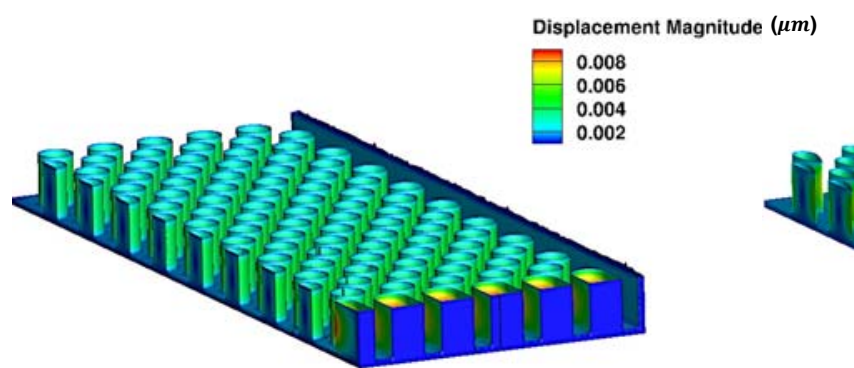

a)

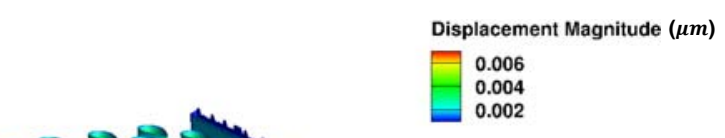

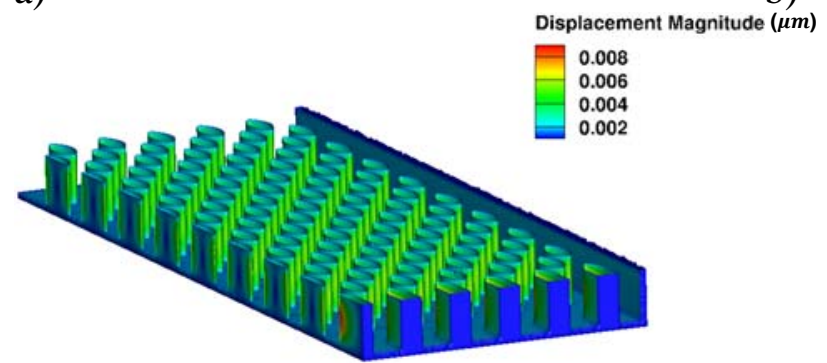

c)

Figure 19. Displacement field due to hydrodynamic and thermal loads on Pareto optimized pin fin having: a) circular, b) airfoil and c) symmetric convex cross section

Figure 20 shows the Von-Mises stress field for the three optimized configurations. It can be seen that the maximum Von-Mises stress ranges from 55 to $85 \mathrm{MPa}$ and are significantly lower than the yield strength of 7000 MPa for silicon [35]. This shows that, from a structural view point, the hydrodynamic loads and thermal loads can be significantly increased without comprimising structual integrity. 


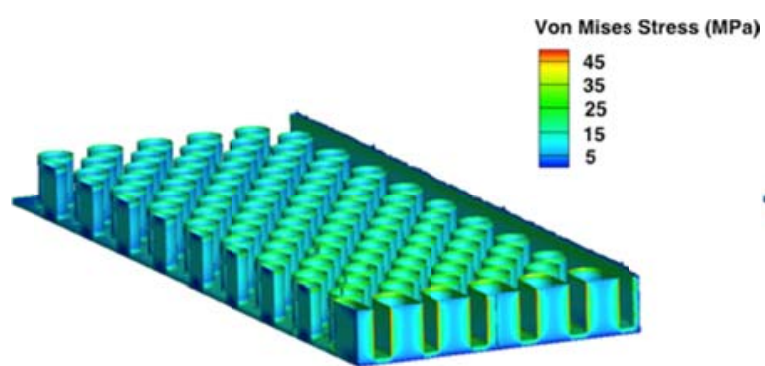

a)

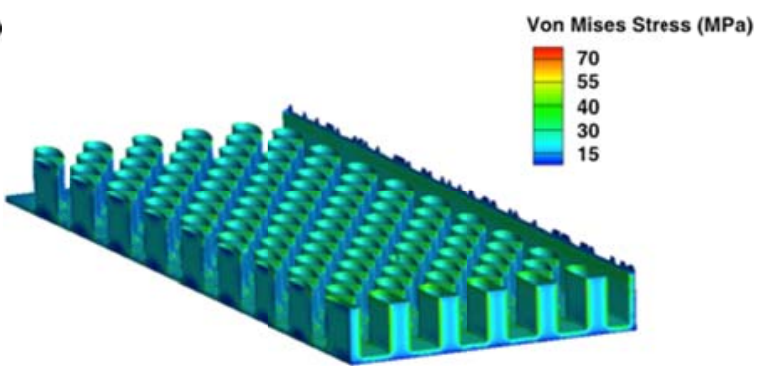

b)

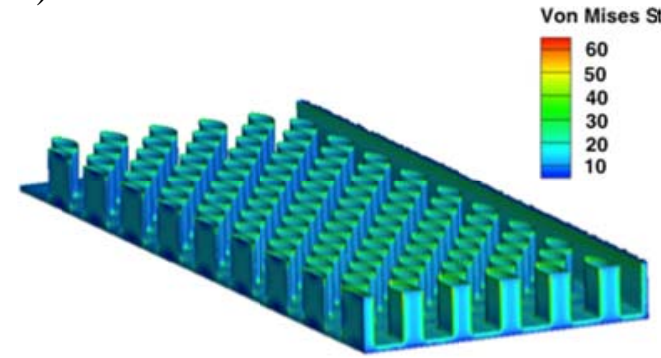

c)

Figure 20. Von-Mises stress distribution due to hydrodynamics and thermal loads on pin fins having: a) circular, b) airfoil and c) symmetric convex cross section

\subsection{Multi-Objective Optimization for Electronic Chips with a Hot Spot}

This section presents the optimization set up and results of the optimization study when applied to design of micro pin-fins with a hot spot. The heat fluxes considered here are beyond those of next generation chips. Similar to the previous sections, this section is divided into problem formulation, un-optimized configurations, optimized configurations and stress-deformation analysis of the optimized configurations. 


\subsubsection{Problem Formulation}

Dissimilar to the previous section, the configurations in this optimization study feature a centrally located hot spot of dimension 0.5 x $0.5 \mathrm{~mm}$. A heat flux of $500 \mathrm{~W} / \mathrm{cm}^{2}$ and $2000 \mathrm{~W} / \mathrm{cm}^{2}$ was imposed on the background and the hot spot, respectively [36]. The same boundary conditions were imposed on the outlet, bottom and sidewalls as in the previous optimization study. The inlet fluid temperature was again kept constant at $30^{\circ} \mathrm{C}$.

The range for design variables, objectives, and constraints are the same as those defined in Section 3.1.2. A total of 30 candidate designs were analyzed to create the response surfaces for the circular pin-fins and a total of 50 designs were analyzed for the airfoil and convex pin-fins. The response surfaces (one for each objective function) were coupled with the NSGA-II algorithm.

\subsubsection{Random Un-Optimized Configurations}

This section presents the random un-optimized configurations with a hot spot created using Sobol's algorithm for comparison with the optimized configurations. Figure 21 shows the temperature field for the three non-optimized configurations. 


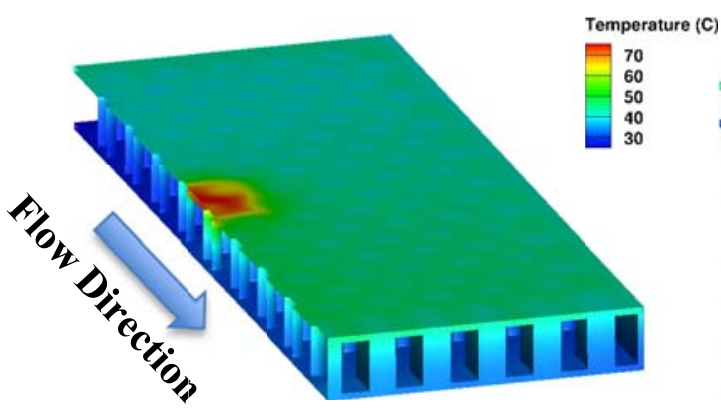

a)

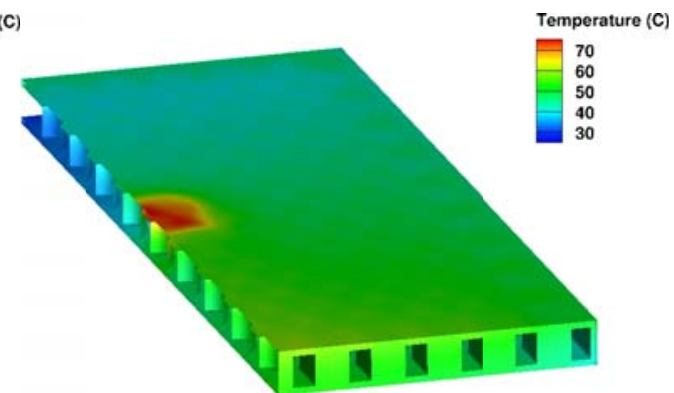

b)

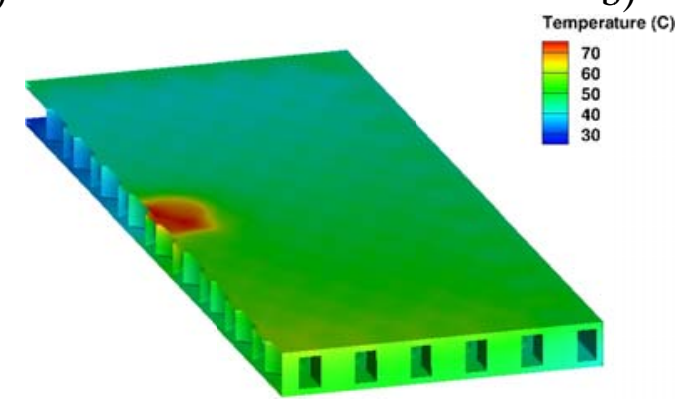

c)

Figure 21. Temperature distribution for the non-optimized configurations of pin fins having: a) circular, b) airfoil, and c) convex cross sections.

Figure 22 shows the pressure field at mid height of each pin-fin configuration. The large stagnation point can be seen at the leading edge of the circular and airfoil geometries. It can be seen that the pressure is much higher than in the case of configurations optimized for uniform heat flux. This is because the higher heat flux at the hot spot requires a greater flow rate to remove the additional thermal energy.

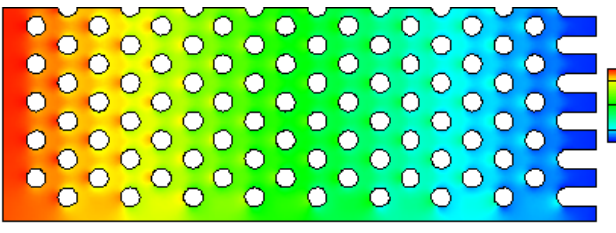

a)

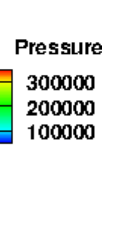

300000 000

\section{8}

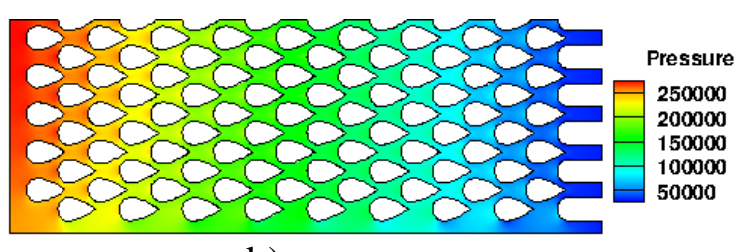

b) 


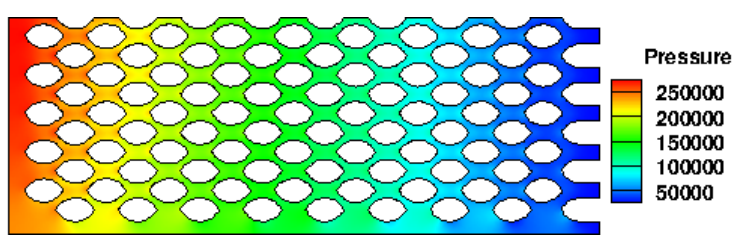

c)

Figure 22. Pressure field for non-optimized configurations of pin fins having: a) circular, b) symmetric airfoil, and c) symmetric convex cross sections.

Figure 23 shows the non-uniform temperature profile on the bottom surface of the chips. It should be noted that the lower temperatures on the bottom surface of the chips indicate that more of the heat is being removed by the fluid via convection.

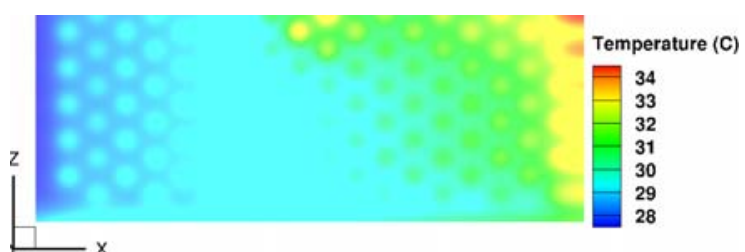

a)

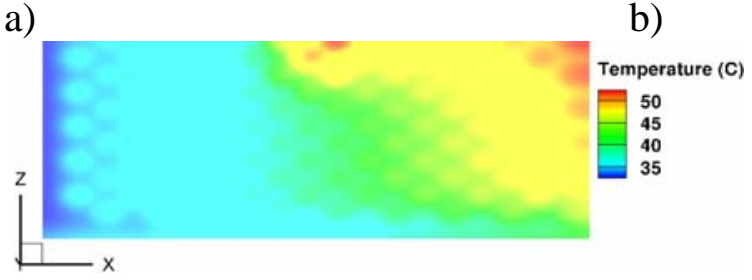

c)

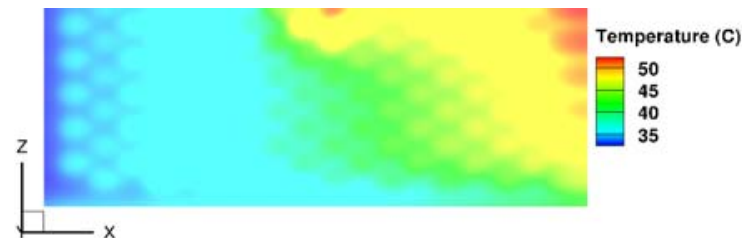

b)

Figure 23. Temperature field on the bottom surface for non-optimized configurations of pin fins having: a) circular, b) symmetric airfoil, and c) symmetric convex cross sections.

\subsubsection{Optimized Configurations}

This section presents the results of the optimization study. For this study, it was found that Hardy’s Multiquadrics RBF outperformed Inverse Quadrics and Gaussian RBF. For this reason, the NSGA-II algorithm was coupled with the Hardy's Multiquadrics RBF. 
Figure 24 represents the Pareto front for the three configurations along with the initial population used to construct the response surface.

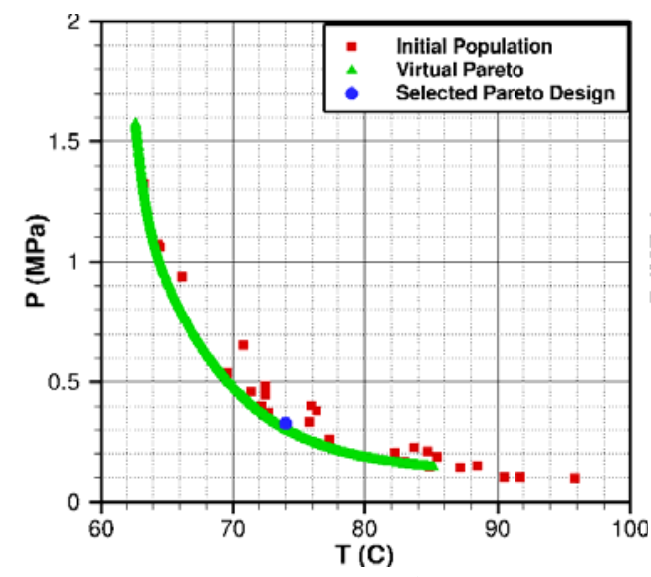

a)

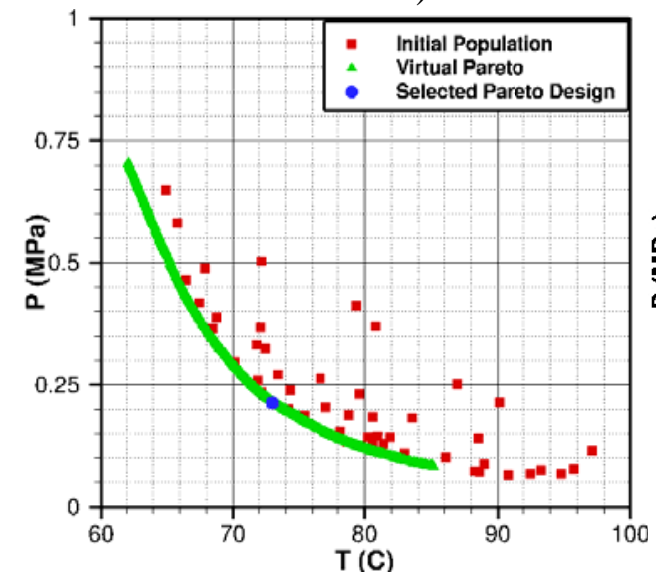

c)

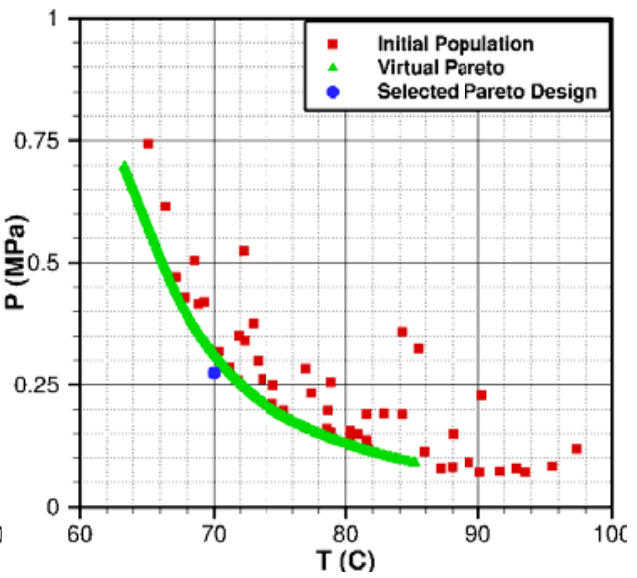

b)

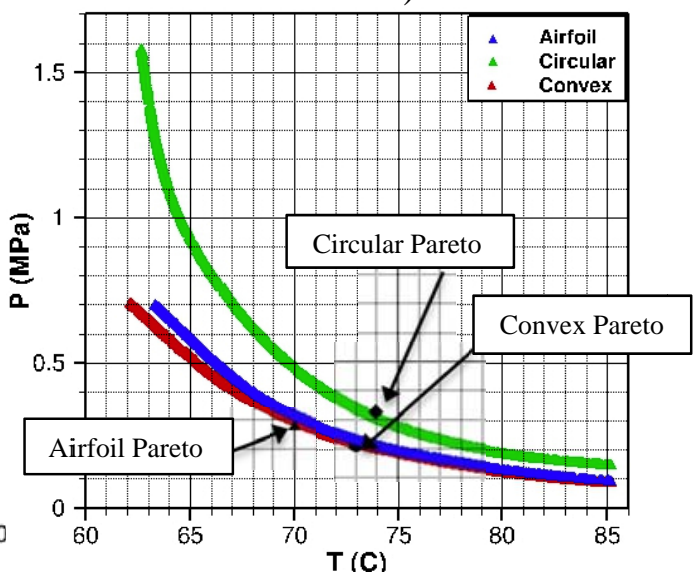

d)

Figure 24. Objective function space for initial population and virtual Pareto designs for array of micro pin-fins having: a) circular, b) symmetric airfoil, c) symmetric convex cross sections, and d) superimposed Pareto frontiers for the three configurations showing that micro pin-fins with convex cross sections offer superior performance

Table 5 shows the geometric parameters and inlet conditions of the three optimized configurations. 
Table 5. Parameters and objective function values for the three Pareto optimized pin fin geometries

\begin{tabular}{c|ccc|}
\hline $\begin{array}{c}\text { Pin-fin Configuration } \\
\text { Parameters }\end{array}$ & Circular & $\begin{array}{c}\text { Symmetric } \\
\text { Airfoil }\end{array}$ & $\begin{array}{c}\text { Symmetric } \\
\text { Convex }\end{array}$ \\
\hline Diameter $(\mu \mathrm{m})$ & 170 & & \\
Chord length $(\mu \mathrm{m})$ & & 280 & 270 \\
Thickness $(\mu \mathrm{m})$ & & 110 & 110 \\
Height $(\mu \mathrm{m})$ & 250 & 250 & 250 \\
Inlet speed $(\mathrm{m} / \mathrm{s})$ & 2.8 & 4.4 & 4.0 \\
\hline Max. Temperature $\left({ }^{\circ} \mathrm{C}\right)$ & 74 & 70 & 73 \\
Inlet pressure $(\mathrm{kPa})$ & 327.77 & 274.42 & 212.55 \\
\hline
\end{tabular}

Figure 25 shows the temperature distribution through the chip. It can be seen that the circular cross section pin fins result in a higher maximum temperature than the airfoil and convex cross section shapes.

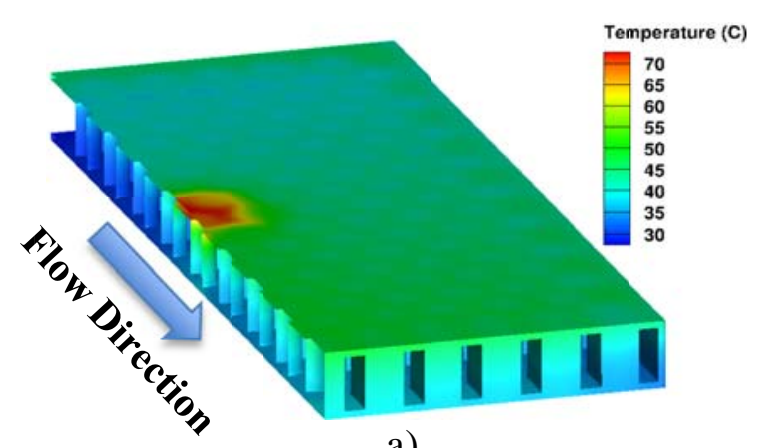

a)

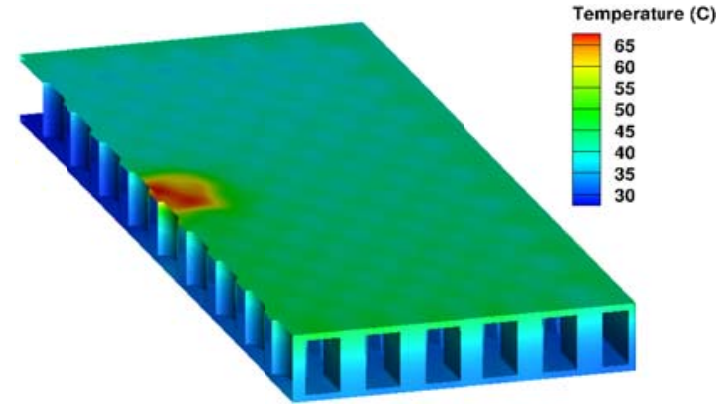

b)

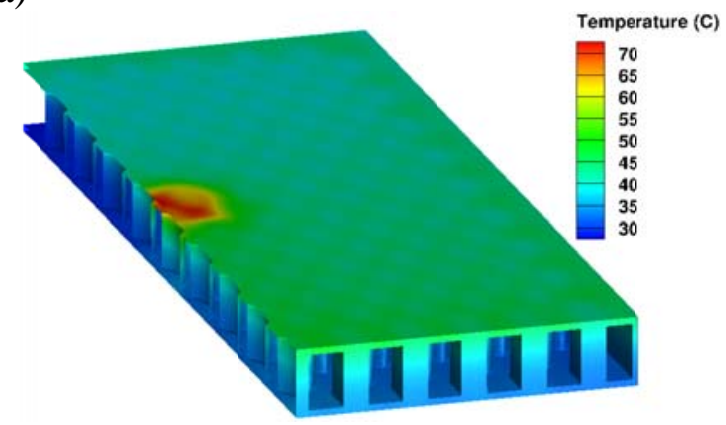

c)

Figure 25. Temperature distribution for the optimized configurations of pin fins having: a) circular, b) airfoil, and c) convex cross sections. 
Figure 25 also shows the higher temperatures at the base of the circular pin-fins. This suggests that the heat is not efficiently being transferred to the fluid, rather it is being carried to the bottom of the chip via conduction. This is not seen in the case of pin-fins having symmetric airfoil and convex shapes as is evident from the lower temperatures near the bottom surface.

Figure 26 shows the temperature distribution on the bottom surface of the three Pareto-optimized configurations. As previously mentioned, the higher temperatures on the bottom surface of the circular cross section pin-fins are due to insufficient convective heat transfer between the pin-fins and the cooling fluid. The higher temperature on the bottom surface of circular cross section pin-fins would require a two-floor configuration [13].

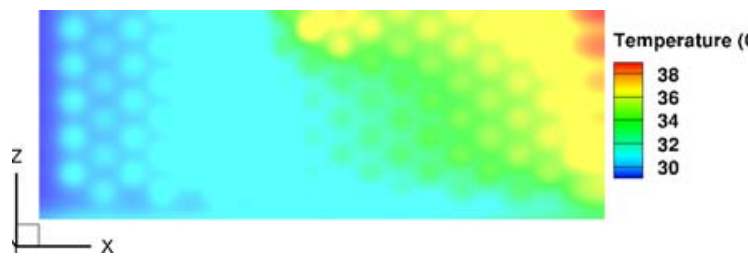

a)

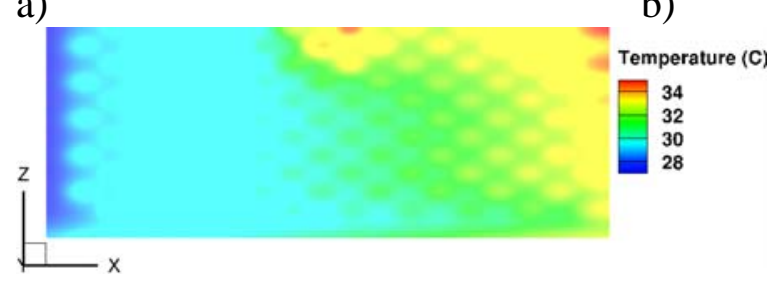

c)

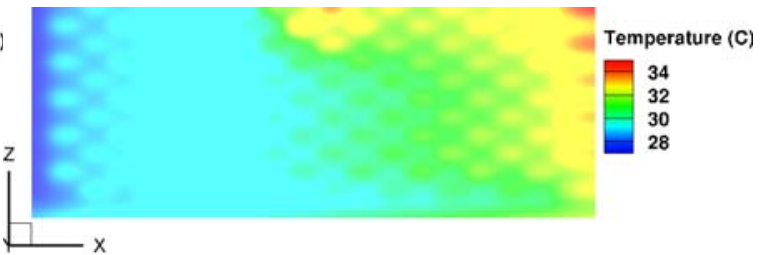

b) Temperature (C) \begin{tabular}{|r|}
\hline 34 \\
\hline 32 \\
30 \\
28 \\
\hline
\end{tabular}

Figure 26. Temperature field on the bottom surface for optimized configurations of pin fins having: a) circular, b) symmetric airfoil, and c) symmetric convex cross sections

Table 6 shows the objective function values for the Pareto-optimized and nonoptimized configurations. It should be mentioned that a different Pareto design may be 
selected from the Pareto front that best satisfies the needed performance. When selecting the presented Pareto designs, priority was given to lower pressure. It can be seen that the Pareto-optimized airfoil and convex cross section pin-fin designs satisfy both objectives better than the circular cross section pin-fins.

Table 6. Objective function values for non-optimized and Pareto optimized arrays of micro pin-fins for each of the three pin fin geometries

\begin{tabular}{lcccc}
\hline & $\left.\mathbf{T ~} \mathbf{(}^{\mathbf{0}} \mathbf{C}\right)$ & $\boldsymbol{\Delta T} \mathbf{( \% )}$ & $\mathbf{p ~ ( k P a )}$ & $\Delta \mathbf{p ~ ( \% )}$ \\
\hline Non-optimized circular & 76 & & 379.89 & \\
Optimized circular & 74 & -3 & 327.77 & -14 \\
Non-optimized airfoil & 77 & & 282.16 & \\
Optimized airfoil & 70 & -9 & 274.42 & -3 \\
Non-optimized convex & 76 & & 261.37 & \\
Optimized convex & 73 & -4 & 212.55 & -19 \\
\hline
\end{tabular}

\subsubsection{Stress-Deformation Analysis of Optimized Configurations}

This section presented the results of the stress-deformation analysis of the Paretooptimized configurations with a hot spot subjected to high thermal loads. All external sides of the chip were fixed so as to investigate the stresses and deformation on the pin fins. Figure 27 shows the displacement field for each of the configurations. Despite the increased thermal loads and hydrodynamic loads, the maximum displacement remains on the nano-level. The maximum displacement can be seen to occur at the leading edge of the pin-fins at the outlet. This is because of the increased thermal loads due to the warmer fluid. 


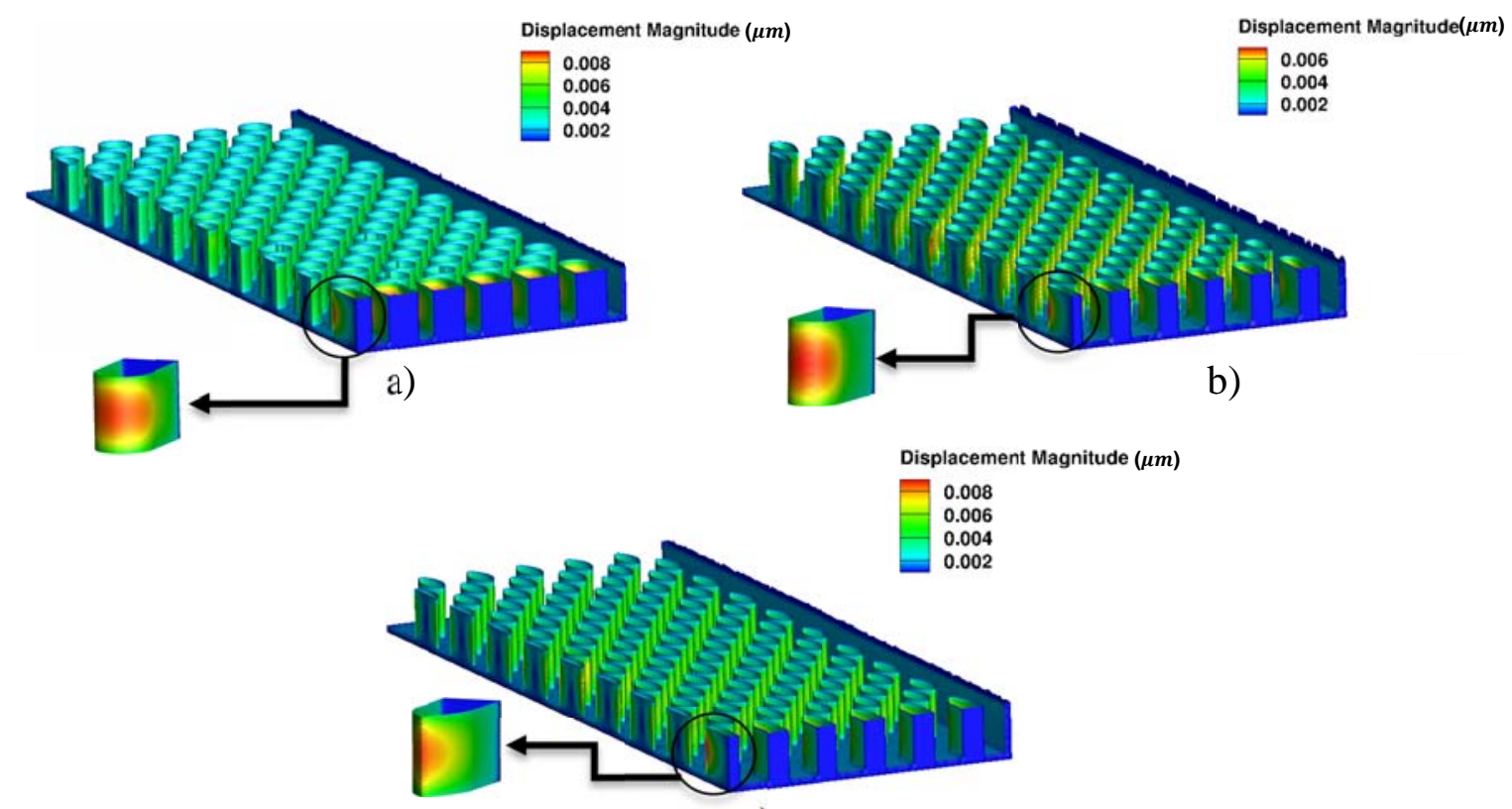

c)

Figure 27. Displacement field due to hydrodynamic and thermal loads on Pareto optimized pin fin having: a) circular, b) airfoil and c) symmetric convex cross section

Figure 28 shows the Von-Mises stress field for the three optimized configurations. It can be seen that the higher stresses are concentrated directly under the hot spot. This is attributed to the significantly increased thermal loads and the fixed boundary between the pin-fins and the rest of the chip. Figure 28 shows that maximum Von-Mises stress for the three configurations ranges from 67 to $97 \mathrm{MPa}$, again being significantly lower than the yield strength of $7000 \mathrm{MPa}$ for silicon. This suggests that, from a structural viewpoint, both thermal and hydrodynamic loads can be further increased without compromising structural integrity. 


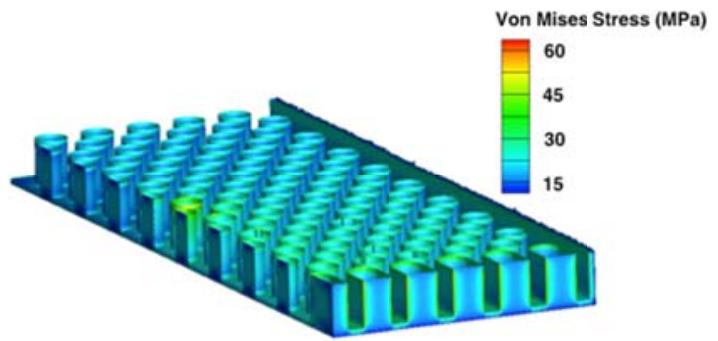

a)

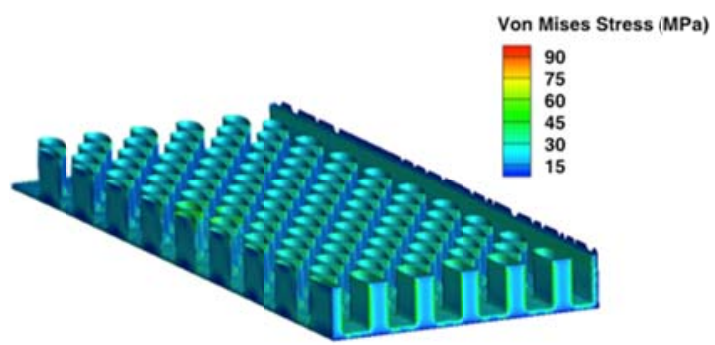

b)

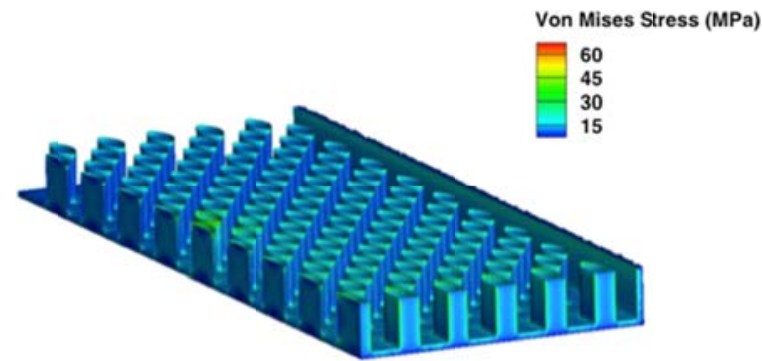

c)

Figure 28. Von-Mises stress distribution due to hydrodynamics and thermal loads on pin fins having: a) circular, b) airfoil and c) symmetric convex cross section 


\section{CHAPTER IV}

\section{INVERSE DESIGN AND PERFORMANCE MAP CONCEPT}

This section presents the setup and the results of the inverse design approach when applied to each of the three pin-fin configurations. The inverse design approach answers the question: Given the inlet coolant temperature, what are the maximum possible background and hot spot heat fluxes that can be imposed without exceeding a specified maximum allowable temperature, while minimizing inlet pressure of the cooling fluid?

\subsection{Problem Formulation}

This inverse design problem can be solved using a multi-objective optimization. The same methodology that was used for direct optimization of the configuration was also applied here with additions to the objective functions. Two additional objectives were incorporated into the existing multi-objective optimization model:

1. Maximize background heat flux

2. Maximize hot spot heat flux

Rather than minimizing the maximum temperature, it was constrained at $85^{\circ} \mathrm{C}$. The inlet cooling fluid temperature in this optimization study was constrained at $30.85^{\circ} \mathrm{C}$. The outlet was again pressurized at $20 \mathrm{kPa}$ gauge. Table 7 shows the design variables being optimized, their ranges and the step sizes used in this study. 
Table 7. Range of design parameters and increment sizes.

\begin{tabular}{|c|c|c|}
\hline Design variable & Range & Step size \\
\hline Pin-fin diameter $(\mu m)$ & $100-200$ & 10 \\
\hline Pin-fin chord length $(\mu m)$ & $200-300$ & 10 \\
\hline Pin-fin thickness $(\mu \mathrm{m})$ & $80-160$ & 5 \\
\hline Pin-fin height $(\mu m)$ & $100-250$ & 50 \\
\hline Coolant inlet speed (m/s) & $1-8$ & 0.1 \\
\hline $\begin{array}{l}\text { Background heat flux } \\
\left(\mathrm{W} / \mathrm{cm}^{2}\right)\end{array}$ & $650-2000$ & 5 \\
\hline Hot spot heat flux $\left(\mathrm{W} / \mathrm{cm}^{2}\right)$ & $2300-5000$ & 10 \\
\hline
\end{tabular}

Various response surface methods, including RBF, Kriging and Gaussian Process were investigated, with Hardy's Multiquadrics outperforming the rest. The objective function values from the response surface deviated by $2 \%$ from those obtained from the conjugate heat transfer. The response surface was created using 120 candidate micro pinfin designs.

\subsection{Results of the Inverse Design}

The results of the optimization study are presented in this section. Figure 29 shows the Pareto front obtained by coupling NSGA-II and response surface. It can be seen that the points on the Pareto front are scattered. This is because discrete values of the design variables are used. Should the design variables be continuous, the Pareto front will have a more defined shape. 


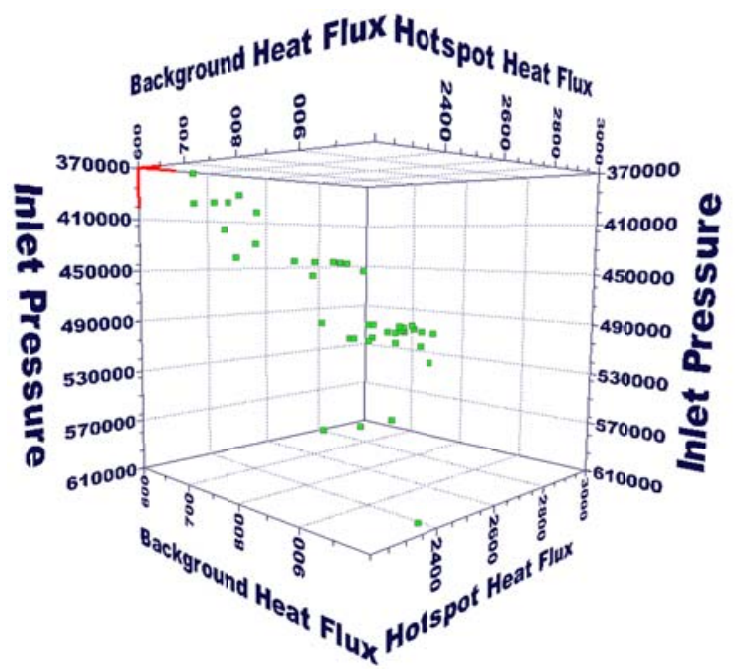

a)

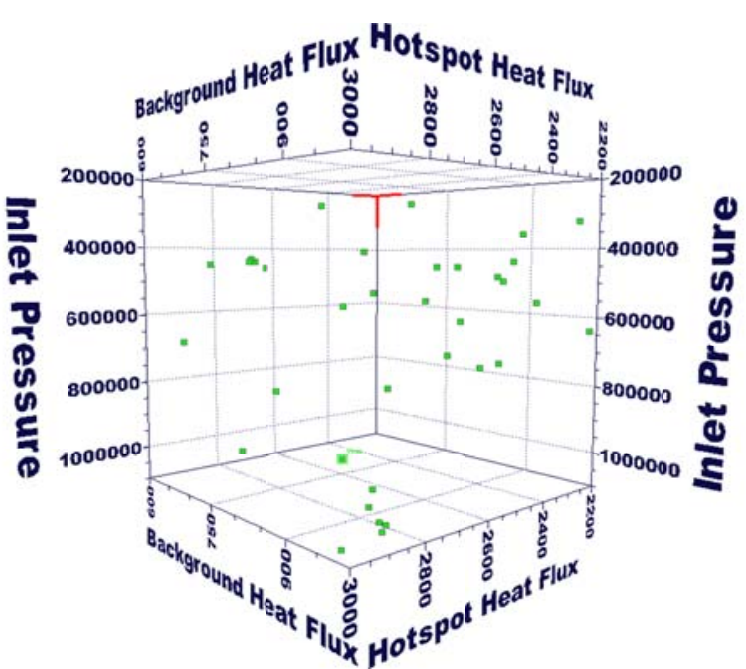

b)

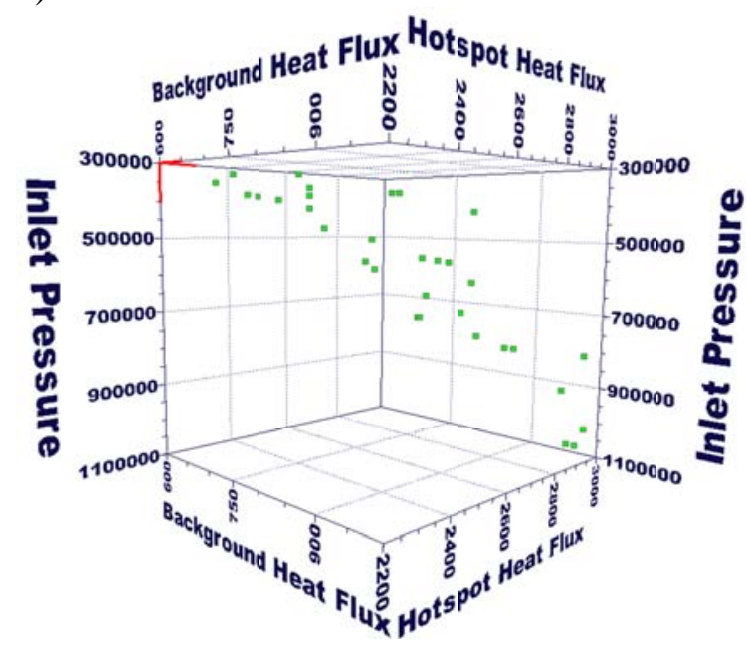

c)

Figure 29. Three-dimensional Pareto frontiers for arrays of pin fins having: a) circular, b) airfoil and c) symmetric convex cross section

Table 8 shows the geometric parameters, inlet conditions and objective function values for the three optimized configurations of pin-fins. It can be seen that the convex cross section pin-fins allow for higher background heat flux application, while requiring minimum mass flow rate. It shows that the airfoil cross section pin-fins can better withstand a higher hot spot heat flux. Those Pareto-optimal designs were chosen for comparison purposes that have similar inlet pressure. 
Table 8. Parameters and boundary conditions for Pareto optimized configurations

\begin{tabular}{llll}
\hline \multicolumn{1}{c}{ Pin-fin cross section } & Circular & Airfoil & Convex \\
\hline Pin-fin diameter $(\mu \mathrm{m})$ & 120 & & \\
Pin-fin chord length $(\mu \mathrm{m})$ & & 280 & 220 \\
Pin-fin thickness $(\mu \mathrm{m})$ & & 120 & 130 \\
Pin-fin height $(\mu \mathrm{m})$ & 250 & 200 & 250 \\
Coolant inlet speed $(\mathrm{m} / \mathrm{s})$ & 6.1 & 4.7 & 4.6 \\
\hline Background heat flux & 805 & 760 & 995 \\
$\left(\mathrm{~W} / \mathrm{cm}^{2}\right)$ & & & \\
Hot spot heat flux $\left(\mathrm{W} / \mathrm{cm}^{2}\right)$ & 2200 & 2540 & 2240 \\
Coolant inlet pressure $(\mathrm{kPa})$ & 395.171 & 409.746 & 393.390 \\
\hline
\end{tabular}

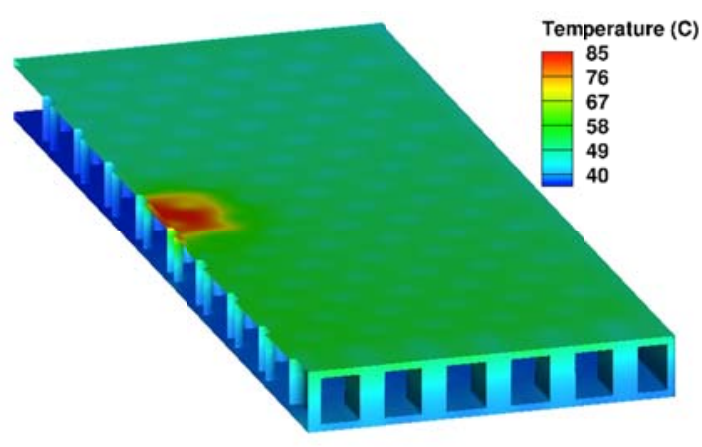

a)

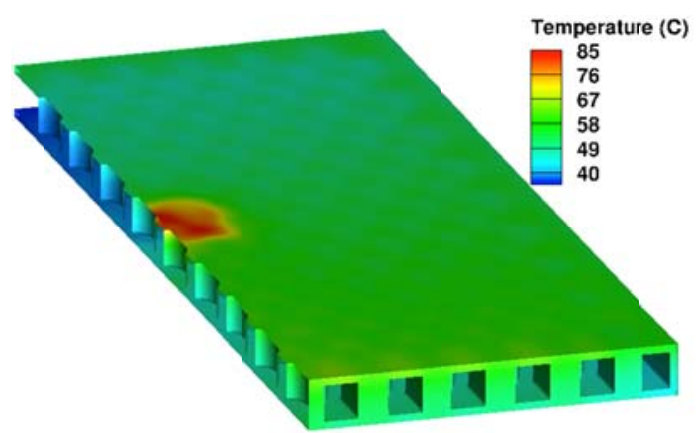

b)

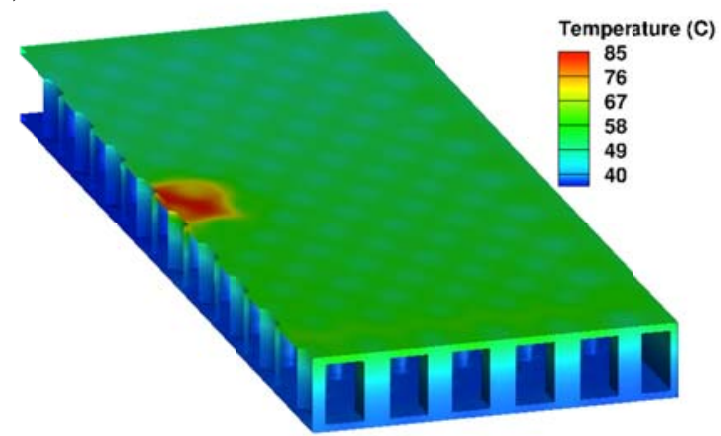

c)

Figure 30. Temperature distribution for the optimized configurations of pin fins having: a) circular, b) airfoil, and c) convex cross sections. 
Figure 30 shows the temperature distribution for the three selected Pareto-optimized designs. It can be seen that for the symmetric airfoil cross section pin-fin configuration, the temperatures can further be decreased using a two-floor configuration.

The temperature distribution on the bottom surface of the chip, shown in Figure 31, also suggests that the heat flux can be further increased on the configuration having symmetric airfoil cross section pin-fins if a two-floor scheme is applied. The higher temperatures on the bottom surface of the airfoil are also due to more heat being conducted through pin-fins, due the increased cross sectional areas resulting from the optimization process. The temperature distribution is similar in the case of circular and convex pin fins.

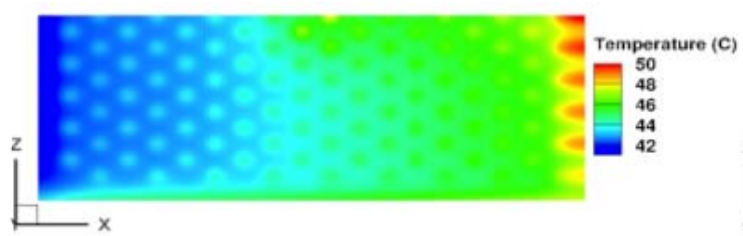

a)

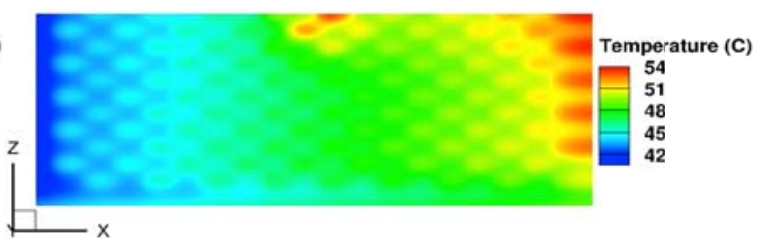

b)

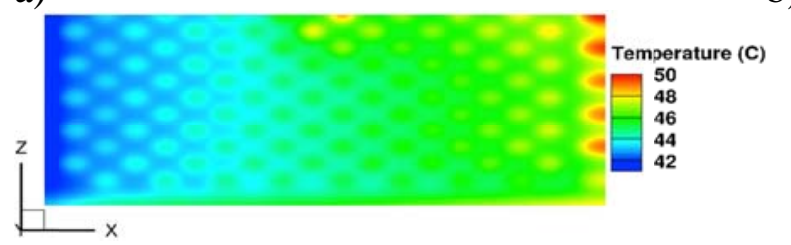

c)

Figure 31. Temperature field on the bottom surface for optimized configurations of pin fins having: a) circular, b) symmetric airfoil, and c) symmetric convex cross sections

Figure 32 shows the pressure distribution at mid-height of the three micro pins-fin configurations. Again, the high pressure stagnation point can be seen in the case of symmetric airfoil and circular cross section pin-fins. It can be reported that nowhere in the fluid domain did the pressure drop to the vaporization pressure of water. 


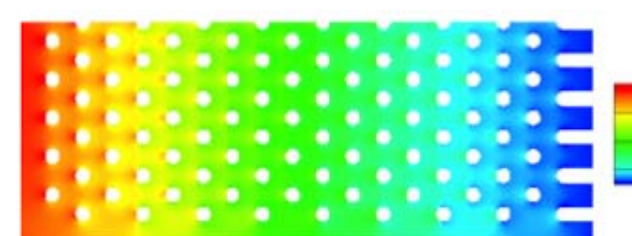

a)

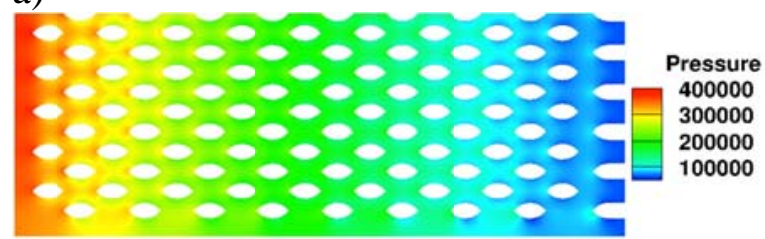

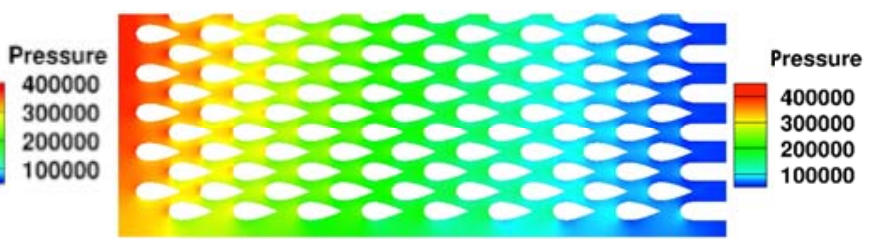

b)

c)

Figure 32. Pressure field for optimized configurations of pin fins having: a) circular, b) symmetric airfoil, and c) symmetric convex cross sections 


\section{CHAPTER V}

\section{CONJUGATE ANALYSIS OF PIN-FIN ARRAYS WITH HEAT SPREADERS}

The effects of thickness and material of a thin film coating on temperature distribution is presented in this section. The thin film heat spreader can be used on the top (hot) surface of the silicon chip. Two different materials, diamond and graphene nanoplatelets, were investigated. Temperature variations along two sample lines are shown for each case. The effect on temperature uniformity of each scheme is also discussed.

\subsection{Problem Formulation}

Thin film heat spreaders are often used to dissipate the heat away from the hot spot and consequently to lower the maximum temperature peak at the hot spot. In this study the effects of diamond and graphene nano-platelets on each of the three pin-fin configurations were investigated [37].

Table 9. Geometric parameters and boundary conditions used for each analysis

\begin{tabular}{llll}
\hline Pin-fin cross section & Circular & Airfoil & Convex \\
\hline Pin-fin diameter $(\mu \mathrm{m})$ & 120 & - & - \\
Pin-fin chord length $(\mu \mathrm{m})$ & - & 280 & 220 \\
Pin-fin thickness $(\mu \mathrm{m})$ & - & 120 & 130 \\
Pin-fin height $(\mu \mathrm{m})$ & 250 & 200 & 250 \\
\hline Coolant inlet speed $(\mathrm{m} / \mathrm{s})$ & 5.1 & 5.5 & 4.8 \\
Background heat flux $\left(\mathrm{W} / \mathrm{cm}^{2}\right)$ & 705 & 875 & 665 \\
Hot spot heat flux $\left(\mathrm{W} / \mathrm{cm}^{2}\right)$ & 2200 & 2200 & 2280 \\
\hline
\end{tabular}


The geometric parameters, heat fluxes and inlet velocity for each of the three configurations used are shown in Table 9. The heat flux levels were obtained using the inverse design approach. The outlet pressure and inlet temperature were the same as for the inverse design. The bottom and sidewalls were again thermally insulated.

A comparison of the solution obtained using a grid of 40 and 50 million cells showed a deviation by less than $1 \%$, thereby assuring grid independency. Figure 33 shows the temperature distribution on the top surface of the three pin-fin configurations without any thin film coating. The two sample lines (streamwise and cross-stream) are shown as well.

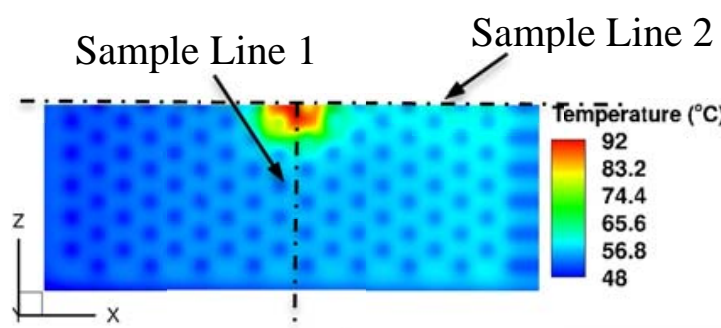

a)

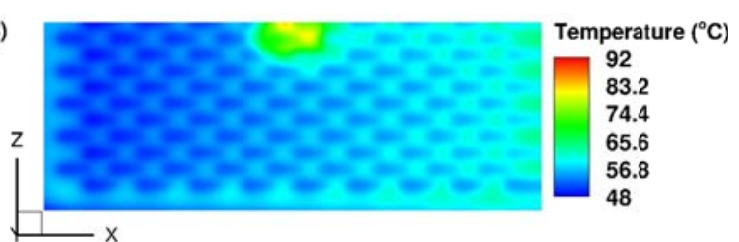

b)

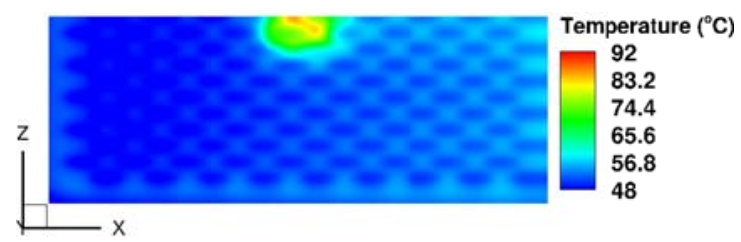

c)

Figure 33. Temperature distribution on the top surface of the top wall of the array of micro pin-fins with: a) circular, b) airfoil and c) convex cross sections and without heat spreader

\subsection{Conjugate Analysis: Diamond Thin Films}

The effects of diamond thin films on the three configurations of micro pin-fins are presented in this section. A total of three thin film thicknesses were investigated for each 
pin-fin configuration: $5 \mu \mathrm{m}, 10 \mu \mathrm{m}$ and $15 \mu \mathrm{m}$. Here, the thermal conductivity of diamond was taken as $1700 \mathrm{Wm}^{-1} \mathrm{~K}^{-1}$. The temperature distribution along the two sample lines is also shown for the three configurations.

Figure 34 shows the temperature distribution on the top surface of the circular pin-fin configuration for the three film thicknesses. It can be seen that the maximum temperature has significantly decreased. It also shows that diamond thin film is very effective in dissipating the heat away from the hot spot, even in the upstream direction.

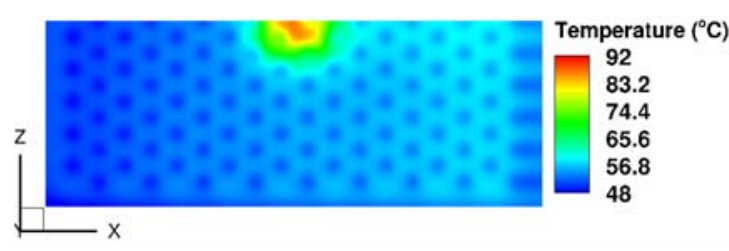

a)

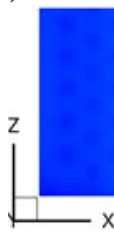

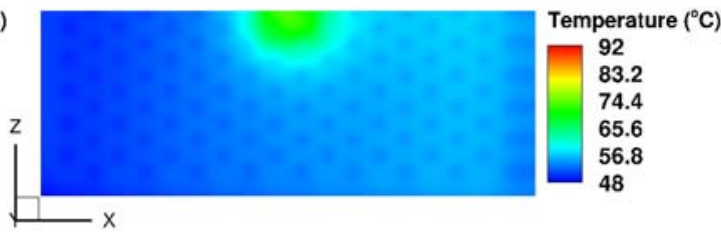

b)

c)

Figure 34. Temperature distribution on the top surface of the top wall of the array of micro-pin fin array having circular cross section pin fins with: a) $5 \boldsymbol{\mu m}$, b) $10 \boldsymbol{\mu m}$ and c) $15 \boldsymbol{\mu} \mathrm{m}$ diamond heat spreader.

Figure 35 shows the temperature distribution along the two sample lines (cross-wise and stream-wise). These results suggest that a thin film thickness exists beyond which further decrease in temperature is no longer possible. Figure 35 also suggests that the diamond thin film has an effective area, outside of which the temperature remains unchanged. It should be mentioned that the oscillations in the temperature are due to the 
presence of pin-fins along the sample lines below the top wall. These pin-fins increase heat conduction in this area, thereby leading to locally reduced temperatures.

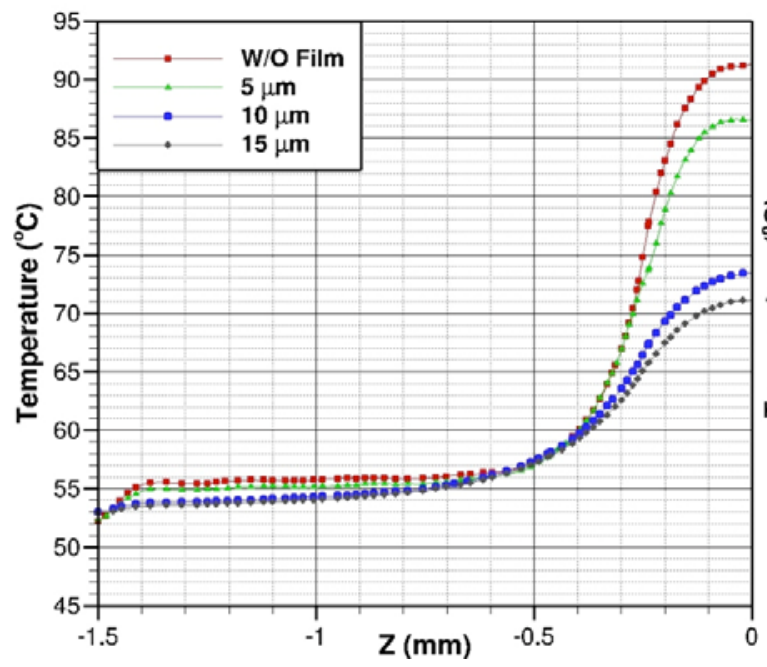

a)

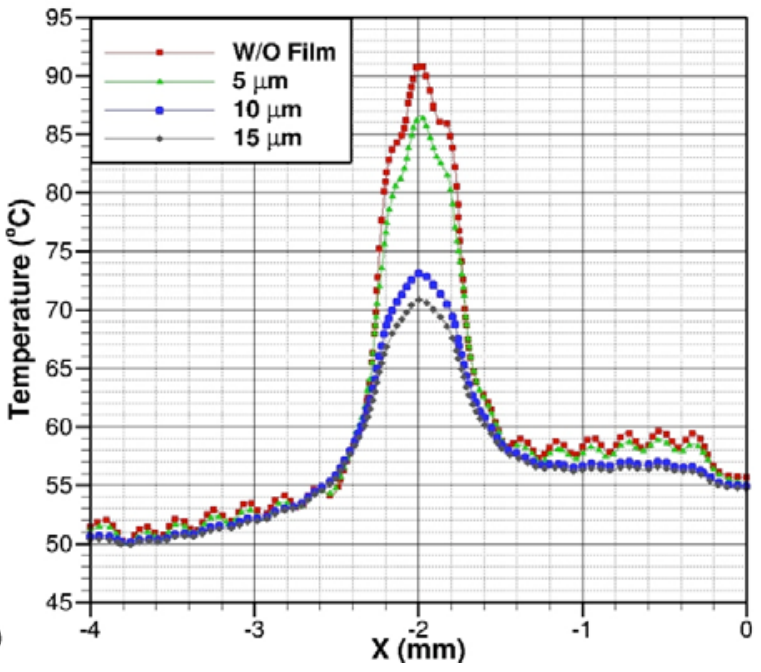

b)

Figure 35. Temperature variation along: a) sample line 1 and b) sample line 2 for the diamond coated top surface of the top wall of an array of micro pin-fins having circular cross sections.

Figure 36 shows the temperature distribution on the top surface of the chip with airfoil cross section pin-fins. The temperature distribution suggests that more heat is being spread in the cross-stream direction than in the stream-wise direction. This improves temperature uniformity, which in turn reduces the thermal stresses.

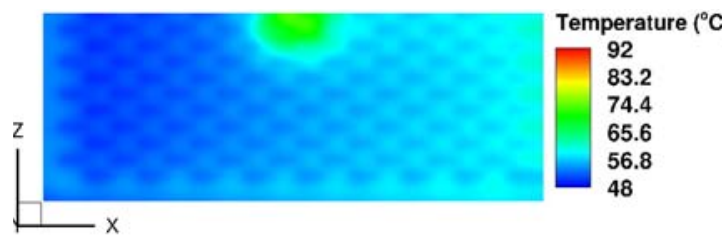

a)

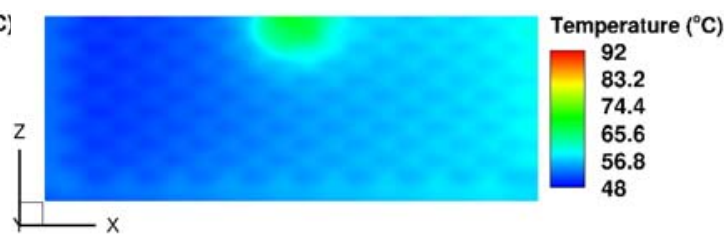

b) 


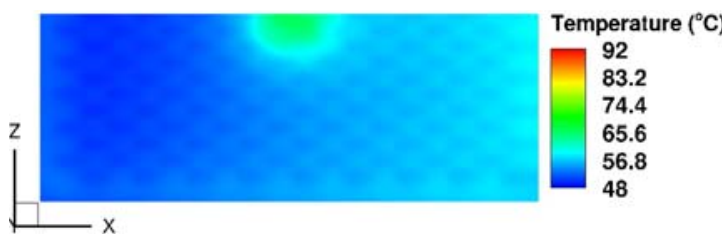

c)

Figure 36. Temperature distribution on the top surface of the top wall of the array of micro-pin fin array having airfoil cross section pin fins with: a) $5 \boldsymbol{\mu m}$, b) $10 \boldsymbol{\mu m}$ and c) 15 $\boldsymbol{\mu} \mathrm{m}$ diamond heat spreader.

Figure 37 shows the temperature along the two sample lines for the micro pin-fin arrays having airfoil cross sections and its top wall coated with a thin diamond film. It can be seen that temperature oscillations have greatly reduced suggesting improved temperature uniformity. The limited area of effectiveness is again seen in this analysis.

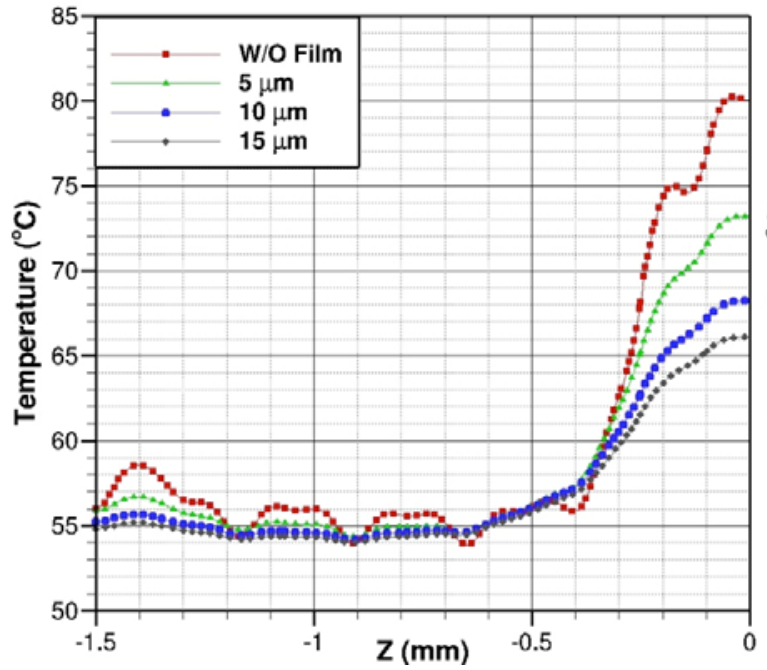

a)

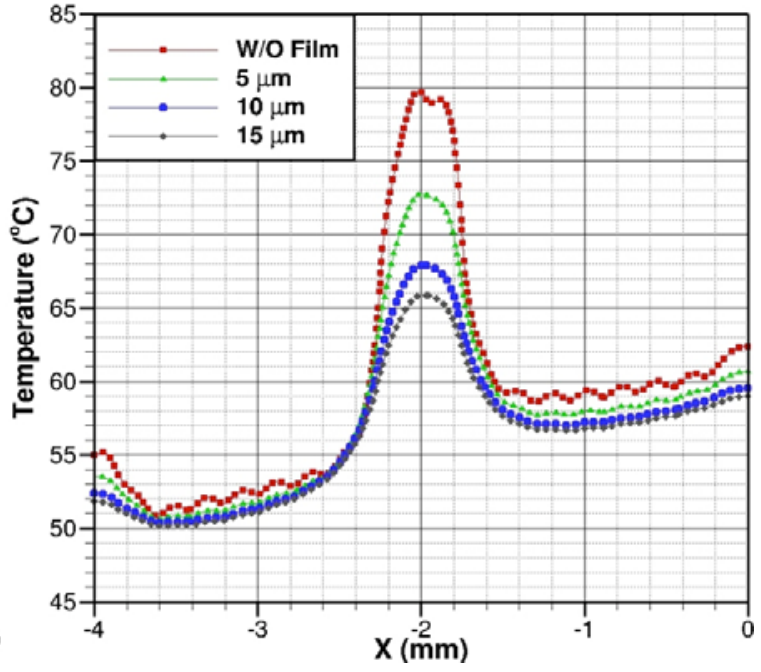

b)

Figure 37. Temperature variation along: a) sample line 1 and b) sample line 2 for the diamond coated top surface of the top wall of an array of micro pin-fins having airfoil cross sections. 


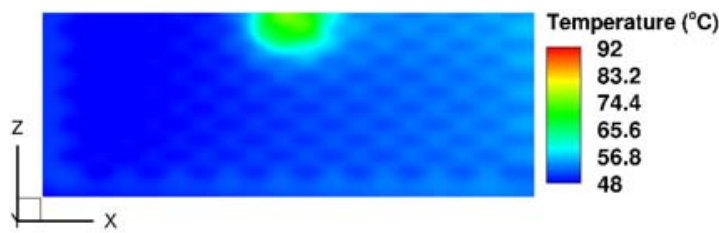

a)

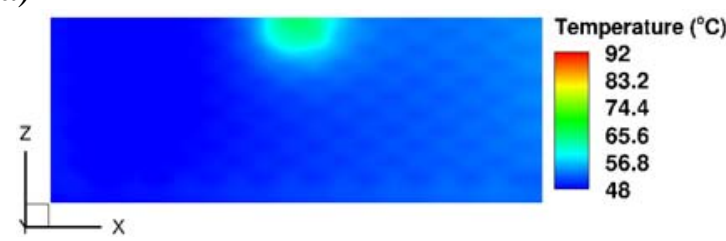

c)

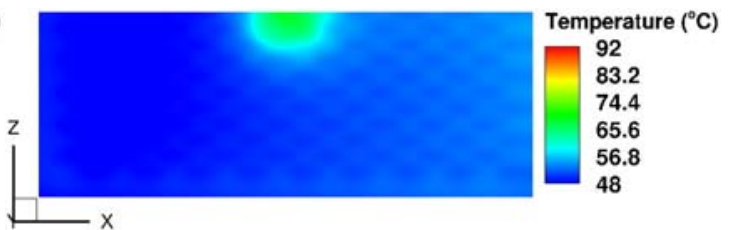

b)

Figure 38. Temperature distribution on the top surface of the top wall of the array of micro-pin fin array having convex cross section pin fins with: a) $5 \boldsymbol{\mu} \mathrm{m}$, b) $10 \boldsymbol{\mu} \mathrm{m}$ and c) $15 \mu \mathrm{m}$ diamond heat spreader.

Figure 38 shows the temperature distribution on the top surface for the convex pin-fin configuration. It shows that more of the heat is being removed in the cross-stream direction as can be seen by the elevated temperatures towards the outlet.

Figure 39 shows the temperature variation along the two sample lines in this case. It can again be seen that with an increase in thin film thickness, the performance gain decays. For all three pin-fin configurations, diamond thin films have shown to also improve local temperature uniformity as can be seen by the absence of temperature oscillations. 


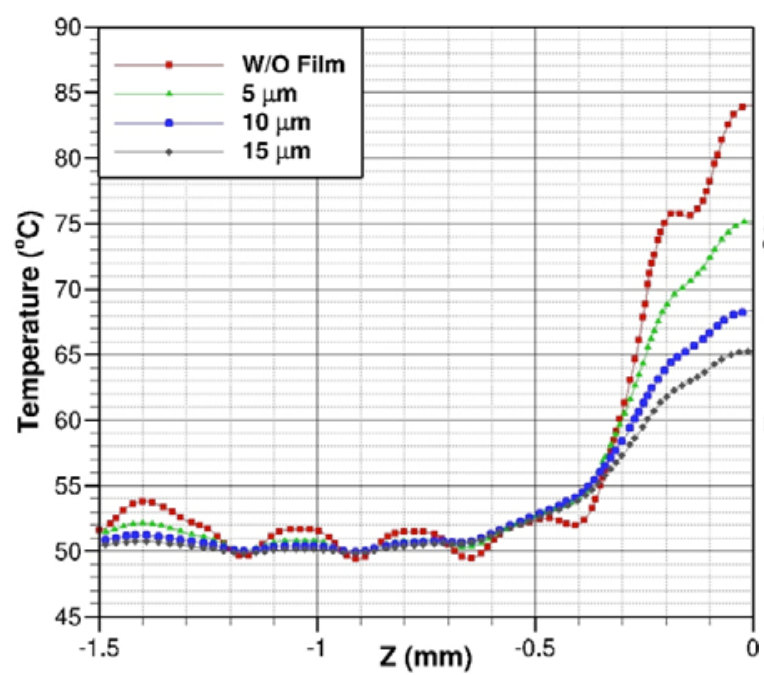

a)

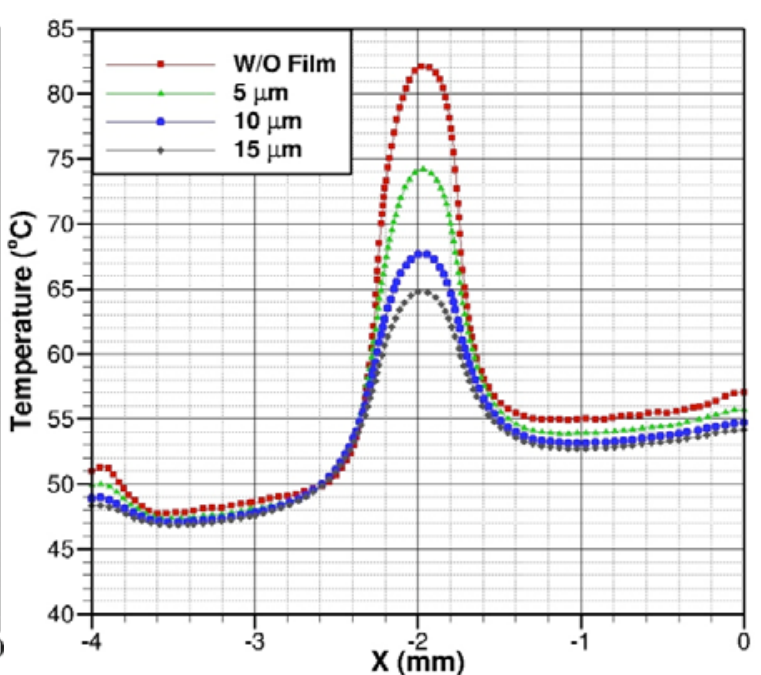

b)

Figure 39. Temperature variation along: a) sample line 1 and b) sample line 2 for the diamond coated top surface of the top wall of an array of micro pin-fins having convex cross sections.

\subsection{Conjugate Analysis: Graphene Nano-Platelets Thin Films}

The effects of Graphene Nano-Platelets on the maximum temperature and temperature distribution are presented in this section. The temperature distribution on the top surface and along the two sample lines is shown for each case. The thermal conductivity of GNP was found to be approximately $5000 \mathrm{Wm}^{-1} \mathrm{~K}^{-1}$. However, this high value of thermal conductivity is only experienced for a single "sheet" or atomic plane of GNP with an approximate thickness of $1 \mathrm{~nm}$. Balandin [38] reported that the thermal conductivity drastically decreases with additional layers of GNP. He stated that for a thin film consisting of more than four atomic planes, the thermal conductivity drops below that of the bulk graphite, but with sufficient number of layers it recovers. Due to the sufficiently thick layers of GNP used in this work, it can be assumed that the thermal 
conductivity of GNP recovers to values of bulk graphite $\left(\mathrm{k}=1400 \mathrm{Wm}^{-1} \mathrm{~K}^{-1}\right)$ [38]. The thermal conductivity of GNP was assumed to be isotropic because of the random orientation of the GNPs arising when creating films of large thicknesses

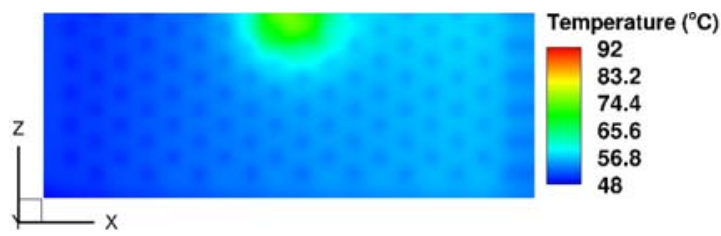

a)

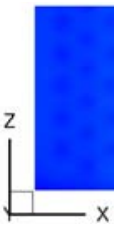

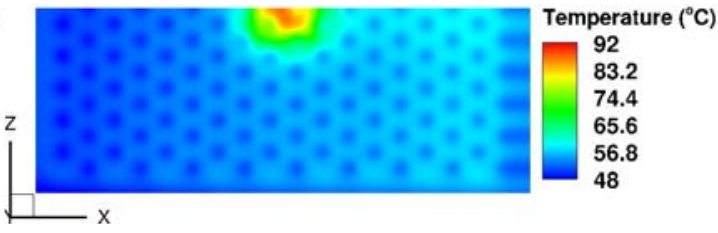

b)

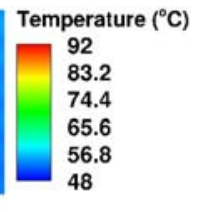

c)

Figure 40. Temperature distribution on the top surface of the top wall of the array of micro-pin fin array having circular cross section pin fins with: a) $5 \boldsymbol{\mu} \mathrm{m}$, b) $10 \boldsymbol{\mu m}$ and c) $15 \mu \mathrm{m}$ graphene nano-platelets heat spreader.

Figure 40 shows the temperature distribution on the top surface of the circular cross section pin-fin configuration. It shows that thin coating does not have a significant effect on either the maximum temperature or its distribution. For significant reduction in the hot spot temperature, thicker coating should be used.

Figure 41 shows the temperature variation along the two sample lines. The large difference between the $5 \mu \mathrm{m}$ and $10 \mu \mathrm{m}$ graphene nano-platelets thin film is evident. It suggests that for any measurable performance gain, a GNP film thickness of more than 5 $\mu m$ is needed. A too thin coating also has insignificant effect on the local and global temperature uniformity. 


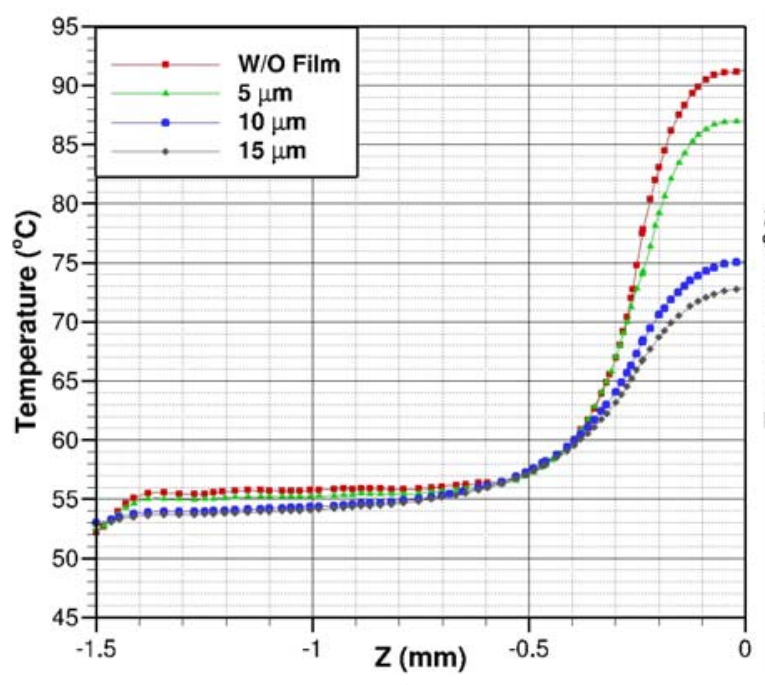

a)

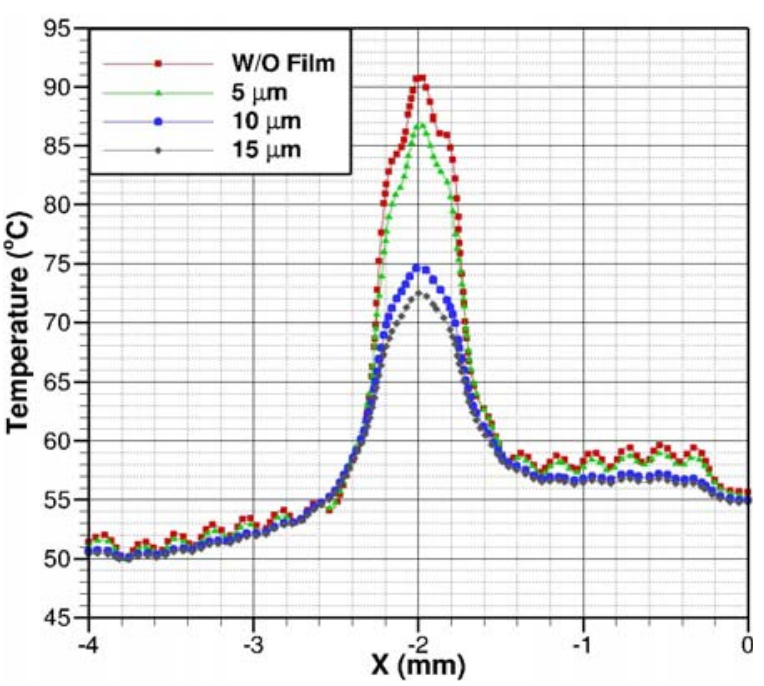

b)

Figure 41. Temperature variation along: a) sample line 1 and b) sample line 2 for the graphene nano-platelets coated top surface of the top wall of an array of micro pin-fins having circular cross sections.

The temperature distribution on the top surface of the chip having pin-fins with symmetric airfoil cross section is shown in Figure 42. It indicates that even the smallest thin film thickness offers significant improvement for the airfoil cross section pin-fin shape over the circular cross section pin-fins. This is shown by the higher temperatures away from the hot spot in the cross-stream direction.

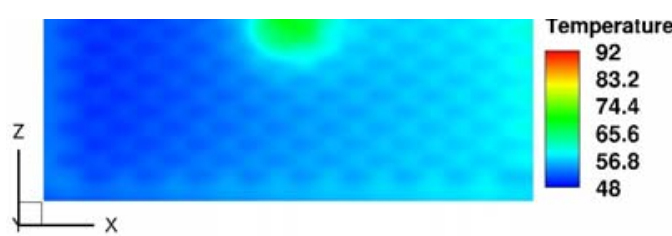

a)

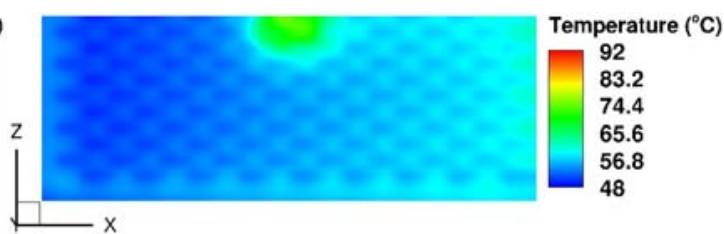

b) 


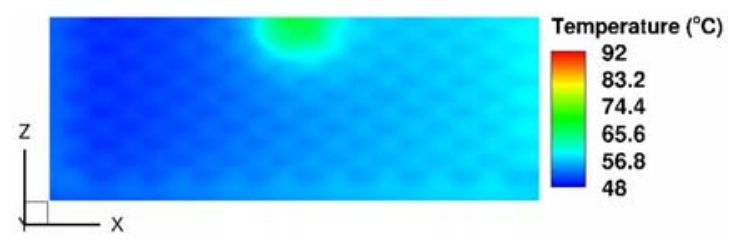

c)

Figure 42. Temperature distribution on the top surface of the top wall of the array of micro-pin fin array having airfoil cross section pin fins with: a) $5 \boldsymbol{\mu} \mathrm{m}$, b) $10 \boldsymbol{\mu m}$ and c) 15 $\boldsymbol{\mu} \mathrm{m}$ graphene nano-platelets heat spreader.

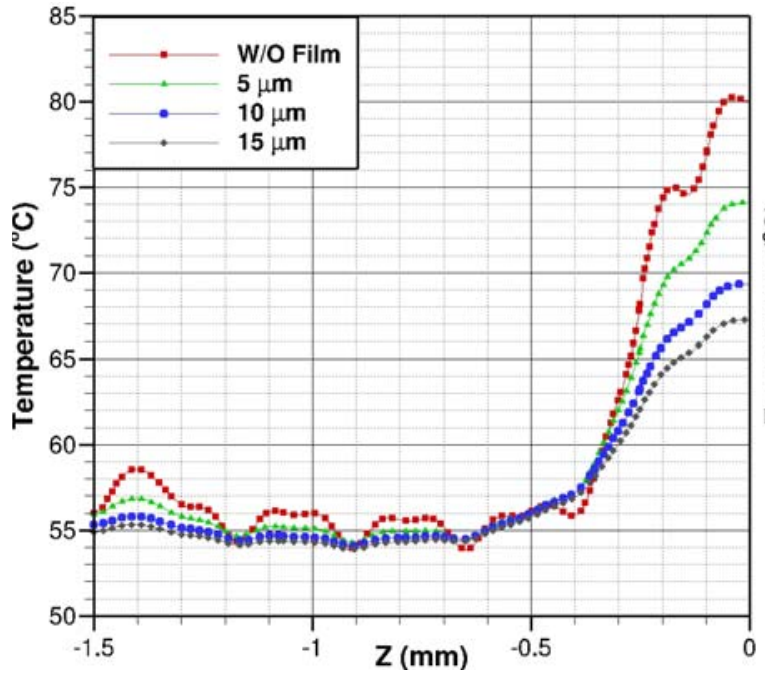

a)

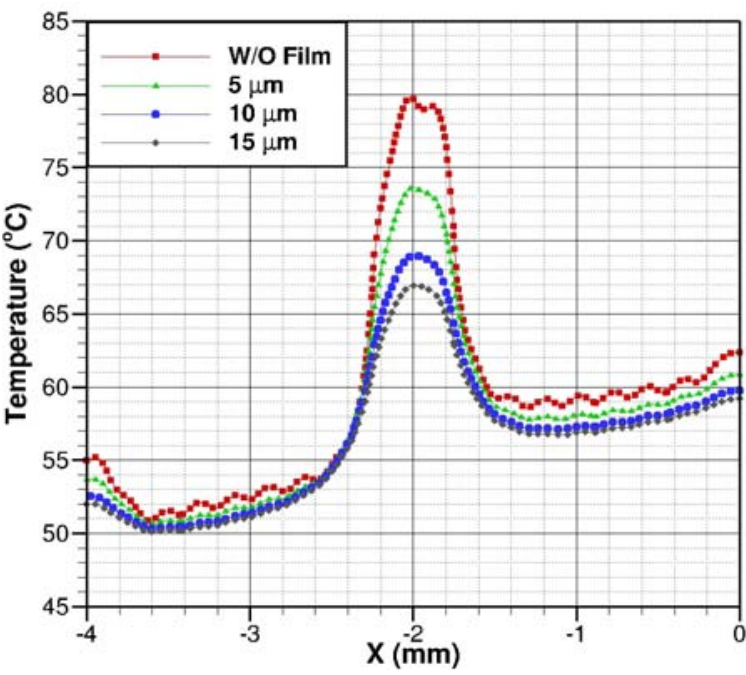

b)

Figure 43. Temperature variation along: a) sample line 1 and b) sample line 2 for the graphene nano-platelets coated top surface of the top wall of an array of micro pin-fins having airfoil cross sections.

The temperature variations along the sample lines, shown in Figure 43, indicate that even the $5 \mu m$ thick heat spreader coating offers significant reduction in the hot spot temperature. As in the case of the diamond thin films, the performance gains of the GNP thin film rapidly decrease as the thickness increases. This conclusion agrees with the findings of Fukatani and Shakouri [18]. 
The temperature distribution for the convex cross section pin-fins coated with GNP heat spreader (Figure 44) is similar to that of the airfoil cross section pin-fin configuration coated with GNP.

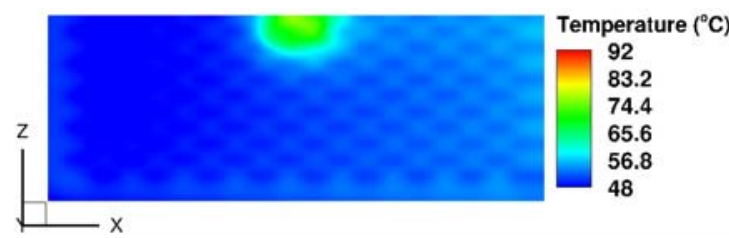

a)

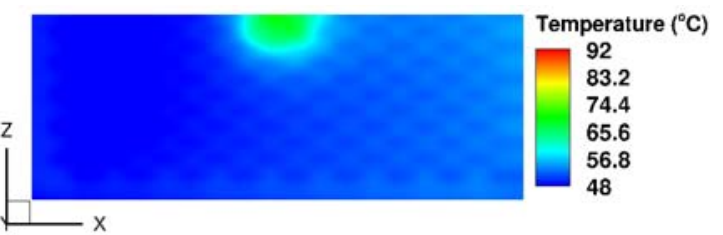

b)

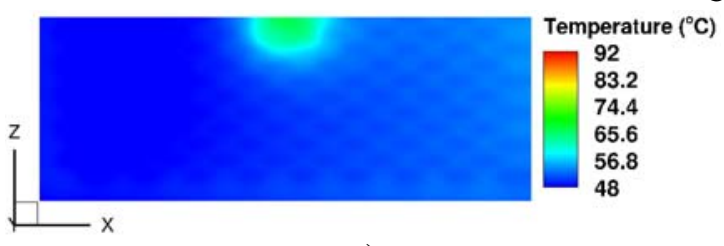

c)

Figure 44. Temperature distribution on the top surface of the top wall of the array of micro-pin fin array having convex cross section pin fins with: a) $5 \boldsymbol{\mu} \mathrm{m}$, b) $10 \boldsymbol{\mu} \mathrm{m}$ and c) $15 \boldsymbol{\mu}$ m graphene nano-platelets heat spreader.

Figure 45 shows the temperature distribution along the two sample lines. It suggests that like the diamond thin film, the GNP thin film also has an effective area outside of which performance gains are insignificant. It also shows that even the thinnest coating improves local temperature uniformity. 


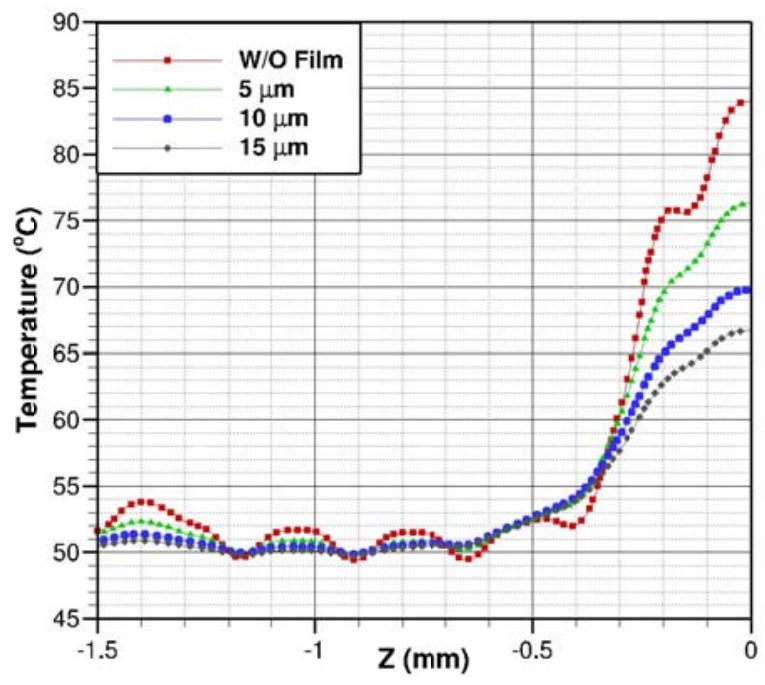

a)

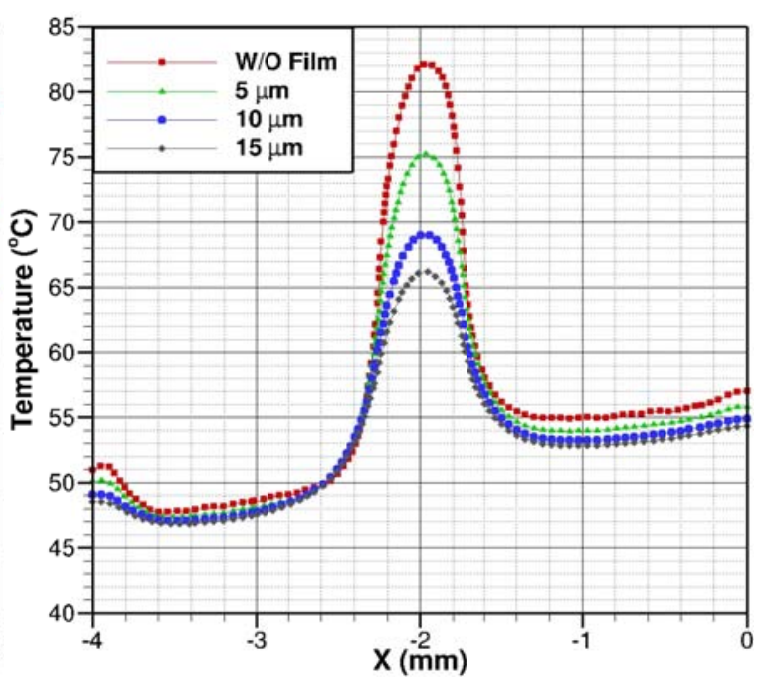

b)

Figure 45. Temperature variation along: a) sample line 1 and b) sample line 2 for the graphene nano-platelets coated top surface of the top wall of an array of micro pin-fins having convex cross sections.

\subsection{Conjugate Analysis: Segmented Thin Films}

It was previously shown that both the diamond and graphene nano-platelet thin films have an effective area outside of which the performance gains are negligible.

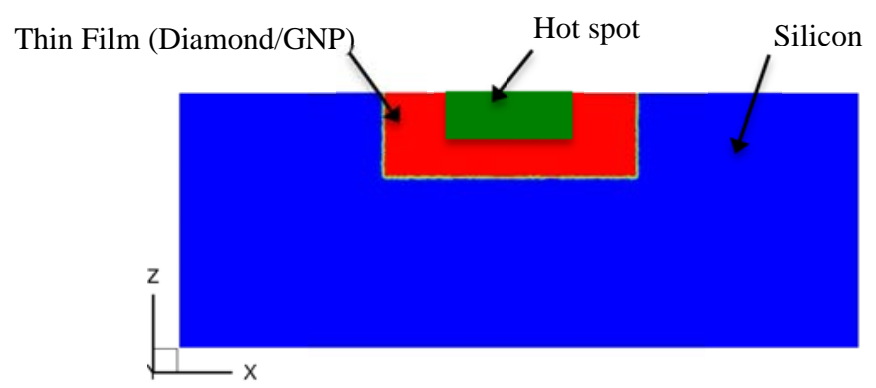

Figure 46. View of the top surface of the electronic chip showing partial coating using thin films 
In this section, the effect of restricting the thin film over the hot spot and slightly beyond the hot spot boundaries is investigated. The thin film was extended $0.25 \mathrm{~mm}$ from the boundaries of the hot spot (Figure 46). The thin film thickness for both materials was kept constant at $15 \mu m$.

Figure 47 shows the temperature distribution on the top surface of the three pin-fin configurations partially coated with diamond thin film. When compared to the fully coated top surface, it can be seen that the partially coated thin film significantly reduces the hot spot temperature. However, it has very little effect on the temperature uniformity. This is because the partially coated thin film is not able to effectively dissipate heat away from the hot spot to cooler regions.

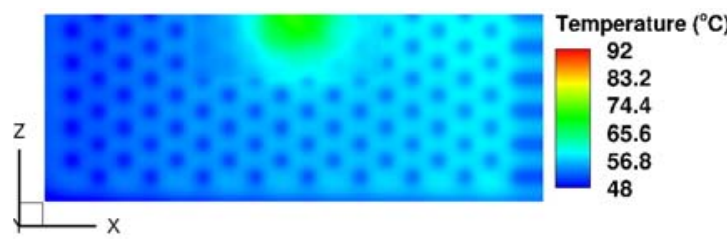

a)

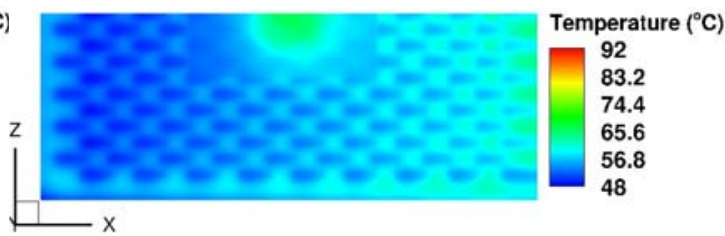

b)

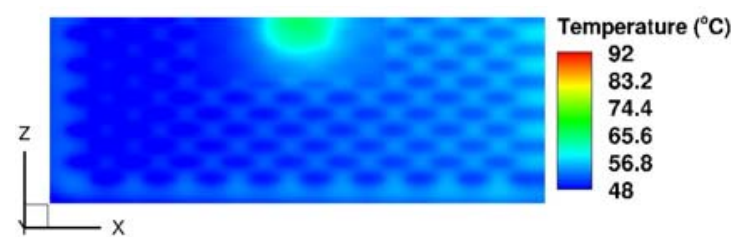

c)

Figure 47. Temperature distribution on the top surface of the top wall of the array of micro-pin fin array with: a) circular, b) airfoil and c) convex cross section and with segmented diamond heat spreader

Figure 48 shows the temperature distribution on the top surface of the three pin-fin configurations when partially coated with GNP. It can again be seen that the segmented thin film can significantly lower the maximum temperature at the hot spot, but that the 
non-uniform temperature distribution has been insignificantly affected. This suggests that although coating only a small region of the chip can reduce material cost, it cannot significantly decrease temperature non-uniformity.

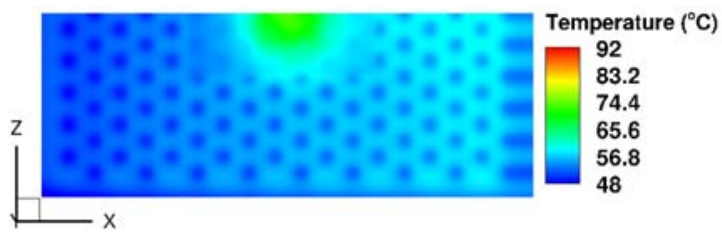

a)

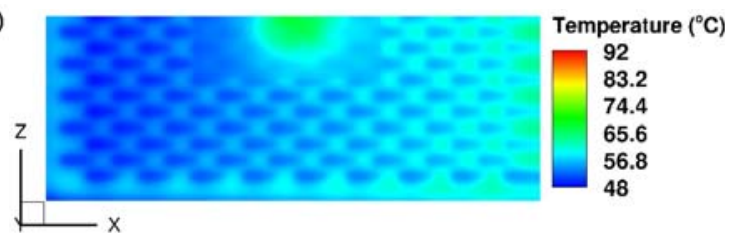

b)

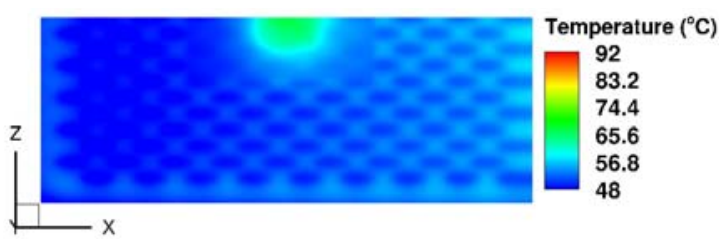

c)

Figure 48. Temperature distribution on the top surface of the top wall of the array of micro-pin-fins with: a) circular, b) airfoil, and c) convex cross section and with segmented graphene nano-platelets heat spreader

Figure 49 shows the temperature distribution along the two sample lines for fully and partially coated top (hot) surface. In the case of the diamond thin films, the maximum temperature difference between the full coating and partially coating is negligible. It also shows that the full coating significantly improves temperature uniformity as can be seen by the absence of the oscillations. In the case of the GNP coating, a small difference in maximum temperature can be seen between the partially and fully coated configurations.

Figure 49b shows the temperature distribution along the streamwise direction. It can be seen that the fully coated top surface carried heat via conduction further upstream. This allows for the cooling process to begin further upstream as can be seen by the lower temperatures at the leading edge of the chip. 


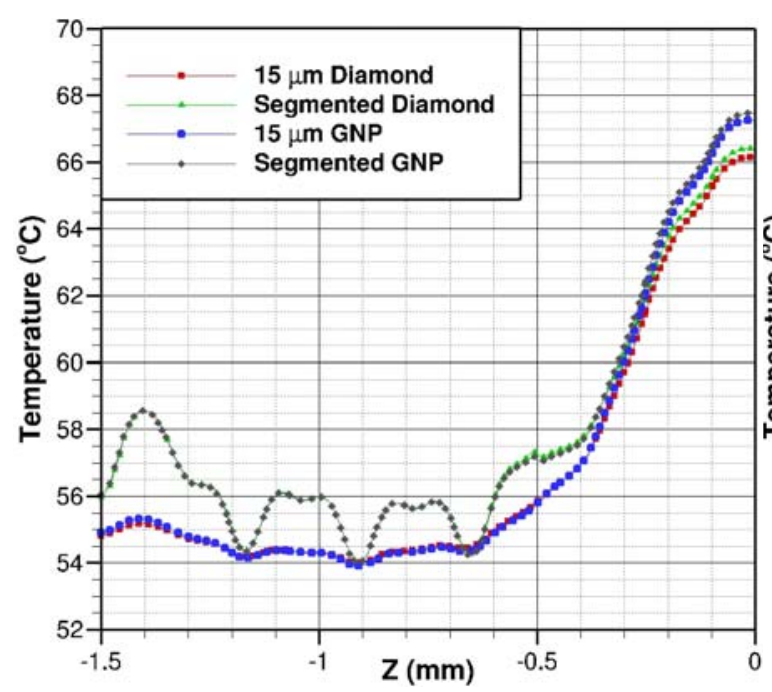

a)

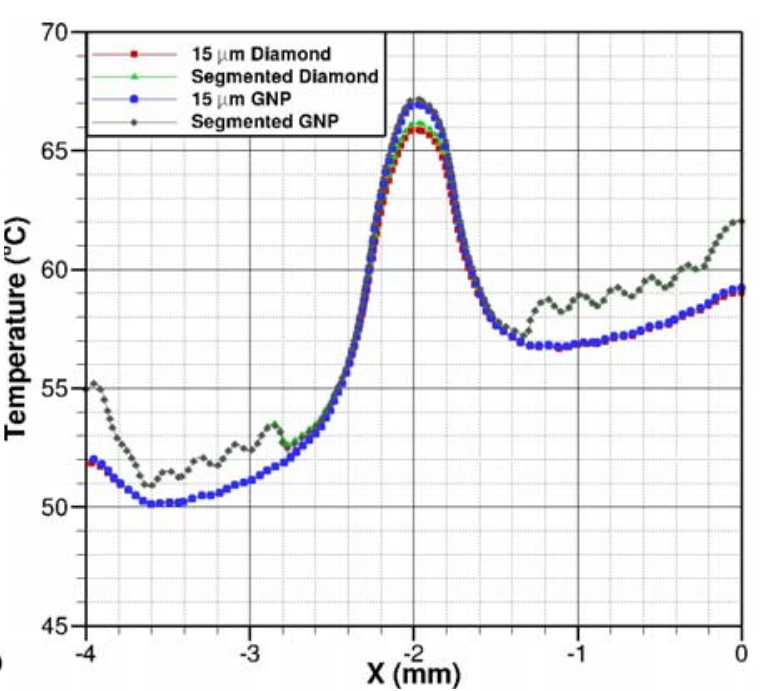

b)

Figure 49. Temperature variation along: a) sample line 1 and b) sample line 2 for array of micro pin-fins having airfoil cross section and fully and partially coated with diamond and graphene nano-platelets.

\subsection{Conjugate Analysis of Thin Film Heat Spreaders: Summary}

The results of the conjugate heat transfer analysis of the thin film heat spreaders are summarized in this section.

Table 10 summarizes the maximum temperature for each of the three pin-fin configurations when the top (hot) surface of the chip is coated with diamond. It can be seen for all three configurations that the maximum temperature decreases as the film thickness increases. It is also apparent that a coating thickness exists after which further increase in thickness will result in insignificant decrease in maximum temperature. 
Table 10. Maximum temperature $\left({ }^{\circ} \mathrm{C}\right)$ for the three pin fin configurations with the top surface of the top wall coated with diamond heat spreader for various film thicknesses.

\begin{tabular}{c|ccc}
\hline \multicolumn{1}{c}{ Thickness } & Circular & Airfoil & Convex \\
\hline No Film & 91 & 82 & 84 \\
$5 \mu \mathrm{m}$ & 86 & 73 & 75 \\
$10 \mu \mathrm{m}$ & 73 & 68 & 68 \\
$15 \mu \mathrm{m}$ & 71 & 66 & 65 \\
Segmented & 71 & 66 & 65 \\
\hline
\end{tabular}

Table 11 summarizes the maximum temperature for each of the three pin-fin configurations when coated with Graphene Nano-Platelets (GNP). When compared to diamond film coating (Table 10), it can be seen (Table 11) that the GNP thin film is just as effective in reducing maximum temperature as the diamond heat spreader, but at lower material costs. The conclusion for diamond heat spreaders also applied for GNP heat spreaders.

Table 11. Maximum temperature $\left({ }^{\circ} \mathrm{C}\right)$ for the three pin fin configurations with the top surface of the top wall coated with graphene nano-platelets heat spreader for various film thicknesses.

\begin{tabular}{c|ccc}
\hline \multicolumn{1}{c}{ Thickness } & Circular & Airfoil & Convex \\
\hline No Film & 91 & 82 & 84 \\
$5 \mu \mathrm{m}$ & 87 & 74 & 76 \\
$10 \mu \mathrm{m}$ & 75 & 69 & 70 \\
$15 \mu \mathrm{m}$ & 63 & 67 & 67 \\
Segmented & 63 & 67 & 67 \\
\hline
\end{tabular}




\section{CHAPTER VI}

\section{CONCLUSIONS}

This section is separated into a summary of the thesis and future work.

\subsection{Summary of Thesis}

Various arrays of micro pin-fins were investigated computationally for their potential to cool the next generation of electronic chips that will have much higher background heat fluxes and a hot spot. Throughout the study, water was used as the heat removing fluid and silicon was used for the solid pin-fins and casing. Three-dimensional conjugate heat transfer analysis was performed on electronic chips having circular, symmetric airfoil and convex cross sections. It was shown that the symmetric airfoil and convex shapes eliminated flow separation that is experienced by the circular pin-fins. It was also shown that both airfoil and convex pin fins not only lead to lower temperature than the circular pin-fins, but also require less pumping power.

For each of the three cross section shapes of the pin-fins, two optimization studies were performed to find an optimum configuration for each of the three shapes under two different operating conditions. The cooling configurations were defined by their geometric parameters and inlet conditions. The three configurations were optimized for two operating conditions:

$>$ Uniform heat flux of $500 \mathrm{~W} / \mathrm{cm}^{2}$ 
Background heat flux of $500 \mathrm{~W} / \mathrm{cm}^{2}$

$>$ A hot spot heat flux of $1000 \mathrm{~W} / \mathrm{cm}^{2}$

The two objectives of both optimization studies were:

\section{$\checkmark$ Minimize maximum temperature \\ $\checkmark$ Minimize inlet pressure (pumping power)}

It was shown that optimized micro pin-fin configurations are capable of cooling next generation heat fluxes. The stress-deformation analysis performed on the optimized configurations showed that the maximum Von-Mises stress is significantly lower than the yield strength of silicon.

An innovative inverse approach that allows for the design of cooling configuration without prior knowledge of operating conditions was also presented and validated. This inverse problem was solved using multi-objective optimization where two more objectives were added to the previous optimization study. The three simultaneous objectives were:

$\checkmark$ Maximize background heat flux

$\checkmark$ Maximize hot spot heat flux

$\checkmark \quad$ Minimize inlet pressure (pumping power)

while constraining the maximum temperature at $85^{\circ} \mathrm{C}$. It was shown that arrays of micro pin-fins with symmetric airfoil cross section are able to cool a background heat flux of $760 \mathrm{~W} / \mathrm{cm}^{2}$ and hot spot heat flux of $2540 \mathrm{~W} / \mathrm{cm}^{2}$. These heat flux levels are beyond that of next generation electronic chips.

To further improve the cooling performance, thin film heat spreaders of varying thickness located on the top (hot) surface of the chip were investigated to further decrease 
the maximum temperature. The two thin film materials investigated included diamond and graphene nano-platelets. It was shown that increasing the thin film thickness leads to lower maximum temperature at the hot spot. It was also shown that an optimum thickness exists beyond which further increase in thin film thickness no longer results in significant performance gains. An observation was made that even the smallest thin film thickness improves both overall and local temperature uniformity. It was noticed that for the circular cross section pin-fins, a thin film thickness of more than $5 \mu \mathrm{m}$ is needed to create any measurable performance. The thin films significantly lower the maximum temperature for all three pin-fin cross section shapes.

\subsection{Future Work}

The following suggestions can be incorporated into future works:

1) More design parameters can be used to define the geometry of the pin-fin cross section shapes and stagger of the pin-fins and incorporated into the optimization.

2) A larger number of suitable objective functions, such as temperature uniformity and manufacturing cost, can be included in the optimization study.

3) The inverse design approach can be applied to configuration with thin films to further increase the allowable heat flux. 
4) Configurations featuring more hot spots can be analyzed and optimized. This would require a robust optimization approach since the location of the hot spots are not usually known.

5) Optimization of two-floor configurations can be performed. It was shown that the temperature on the bottom surface remains relatively high and would require twofloor configuration. 


\section{LIST OF REFERENCES}

[1]. A. Bar-Cohen, "Gen-3 thermal management technology: role of microchannels and nanostructures in an embedded cooling paradigm”, Journal of Nanotechnology in Engineering and Medicine, 4(2), 020907, 2013

[2]. M.A. Ebadian and C.X. Lin, "A Review of High-Heat-Flux Heat Removal Technologies,” Journal of Heat Transfer, Vol 133, 2011, pp. 110801-1 - 110801-11

[3]. W. Qu and I. Mudawar, "Analysis of Three-Dimensional Heat Transfer in MicroChannel Heat Sinks,” International Journal of Heat and Mass Transfer, Vol. 45, 2002, pp. 3973-3985

[4]. A. Abdoli, G.S. Dulikravich, G. Vasquez and S. Rastkar, "Thermo-Fluid-StressDeformation Analysis of Two-Layer Mcrochannels for Cooling Chips with Hot Spots,” Journal of Electronic Packaging, 137(3), 2015. 031003

[5]. A. Abdoli and G.S. Dulikravich, "Multi-Objective Design Optimization of MultiFloor, Counterflow Micro Heat Exchangers," Proccedings of the ASME Heat Transfer Conference Paper HT2013-17738, Minneapolis, MN, USA, July 14-19, 2013; also published in ASME Journal of Heat Transfer, Vol. 136, 2014, pp. 101801-1 - 101801-10

[6]. A. Abdoli and G.S. Dulikravich, "Optimized Multi-Floor Throughflow Micro Heat Exchangers,” ASME IMECE Paper IMECE2013-63590, San Diego, CA, November 15-20, 2013; also published in International Journal of Thermal Sciences, Vol. 78, 2014, pp. 111-123

[7]. M. Fabbri and V.K. Dhir, "Optimized Heat Transfer for High Power Electronics Cooling Using Arrays of Microjets,” ASME Journal of Heat Transfer, Vol. 127, 2005, pp. 760-769

[8]. A. Husain, S. Kim and K. Kim, "Performance Analysis and Design Optimization of Micro-Jet Impingement Heat Sink,” Heat and Mass Transfer, Vol. 49, 2013, pp. 16131624

[9]. F. Alfieri, S. Gianini, M.K. Tiwari, T. Brunschwiler, B. Michel, and D. Poulikakos, Computational modeling of hot-spot identification and control in 3-D stacked chips with integrated cooling, Numerical Heat Transfer Part A, Vol. 65, 2014, pp. 201215.

[10]. F. Alfieri, M.K. Tiwari, A. Renfer, T. Brunschwiler, B. Michel, and D. Poulikakos, Computational modeling of vortex shedding in water cooling of 3D integrated electronics, International Journal of Heat Fluid Flow, Vol. 44, 2013, pp. 745755. 
[11]. A. Kosar, and Y. Peles, "Thermal-hydraulic performance of MEMS-based pin fin heat sink”, ASME Journal of Heat Transfer, Vol. 128, 2006, pp. 121-131.

[12]. S. Ndao, Y. Peles, and M.K. Jensen, "Effects of Pin Fin Shape and Configuration on the Single-Phase Heat Transfer Characteristics of Jet Impingement on Micro Pin Fins”, International Journal of Heat and Mass Transfer, Vol. 70, 2014, pp. 856-863.

[13]. A. Abdoli, G. Jimenez, and G.S. Dulikravich, “Thermo-Fluid Analysis of Micro Pin-Fin Array Cooling Configurations for High Heat Fluxes with a Hot Spot”, International Journal of Thermal Sciences, Vol. 90, 2015, pp. 290-297.

[14]. J.F. Tullius, T.K. Tullius, and Y. Bayazitoglu, "Optimization of Short Micro Pin Fins in Minichannels,” International Journal of Heat and Mass Transfer, Vol. 55, 2012 pp. 3921-3932

[15]. M.J. Gonzalez, N. Jelisavcic, R. Moral, D. Sahoo, G.S. Dulikravich, and T.J. Martin, "Multi-Objective Design Optimization of Topology and Performance of Branching Networks of Cooling Passages,” International Journal of Thermal Sciences, Vol. 46, 2007, pp. 1191-1202

[16]. Y. Zhou and J. Yu, "Design Optimization of Thermoelectric Cooling System for Applications in Electronic Devices,” International Journal of Refrigeration, Vol. 35, 2012, pp. 1139-1144

[17]. A. Husain, K.Y. Kim, "Shape Optimization of Micro-Channel Heat Sink for Micro-Electronic Cooling,” IEEE Componenets and Packaging Technologies, Vol. 31, 2008, pp. 322-330

[18]. K. Fukutani and A. Shakouri, “Optimization of Thin Film Microcoolers for Hot Spot Removal in Packaged Integrated Circuit Chips," 22nd IEEE SEMI-THERM Symposium, Dallas, Texas, pp. 130-134, March 14- 16, 2006.

[19]. P. Wang, P. McCluskey and A. Bar-Cohen, "Hybrid Solid- and Liquid-Cooling Solution for Isothermalization of Insulated Gate Bipolar Transistor Power Electronic Devices," IEEE Transactions on Components, Packaging and Manufacturing Technology, Vol. 3, No. 4, 2013, pp. 601-611

[20]. S.B. Singh, H. Naseem, A. Krishnamoorthy, W.D. Brown and A.P. Malshe, "Thermal Management Using Planarized CVD-Diamond Substrates," The International Journal of Microcircuits and Electronic Packaging, Vol. 23, No.1, 2000, pp. 99- 109

[21]. C. LaBounty, A. Shakouri, G. Robinson, P. Abraham and J.E. Bowers, "Design of Integrated Thin Film Coolers," Proc. of $19^{\text {th }}$ International Conference on Thermoelectrics, ed. D.M. Rowe, Cardiff, UK, August 20-24, 2000. 
[22]. M. Smalc, G. Shives, G. Chen, S. Guggari and J. Norley, "Thermal Performance of Natural Graphite Heat Spreaders,” Paper InterPACK2005-73073, San Francisco, CA, July 17-22, 2005,

[23]. S.B. Pope, “Turbulent Flows,” Cambridge University Press, 2000

[24]. ANSYS Fluent Theory Guide

http://ansys.com/Products/Simulation+Technology/Fluid+Dynamics

[25]. R.B. Hetnarski, M.R. Eslami, "Thermal Stresses - Advanced Theory and Applications,” Publisher Springer Netherlands, ISBN 978-1-4020-9246-6, 2009

[26]. ANSYS Structural Analysis Guide 2009, http://www.ansys.com/Products/Simulation+Technology/Structural+Analysis

[27]. S.V. Patankar,”Numerical Heat Transfer and Fluid Flow,” Taylor and Francis, ISBN 978-0-89116-522-4

[28]. modeFRONTIER, Software Package, 2014, Ver. 4.5.4, ESTECO, Trieste, Italy.

[29]. M.J. Colaco and G.S. Dulikravich, "A Survey of Basic Deterministic, Heuristic and Hybrid Methods for Single-Objective Optimization and Response Surface Generation,” Chapter 10 in Thermal Measurements and Inverse Techniques, (eds. H.R.B. Orlande and O. Fudym, D. Maillet and R. Cotta), Taylor \& Francis, May 2011, pp. 355405

[30]. K. Deb, A. Pratap, S. Agarwal, and T. Meyarivan, "A Fast and Elitist MultiObjective Genetic Algorithm-NSGA-II," KanGAL Report Number 2000001, 2000

[31]. R.L. Hardy, "Multiquadric equations of topography and other irregular surfaces," Journal of Geophysics Research, Vol. 176, 1990, pp. 1905-1915

[32]. M.J. Colaco, W.B. Silva, A.C. Magalhaes, and G.S. Dulikravich, "Response Surface Methods Applied to Scarce and Small Set of Training Points - A Comparative Study, "International Conference on Engineering Optimization, Rio de Janeiro, Brazil, 15 Jun, 2008.

[33]. M. Sobol, "Distribution of points in a cube and approximate evaluation of integrals,” U.S.S.R. Comput. Maths. Math. Phys, 7, 1967, pp. 86-112

[34]. S.R. Reddy and G.S. Dulikravich, "Inverse Design of Cooling of Electronic Chips Subject to Specified Hot Spot Temperature and Coolant Inlet Temperature," Paper InterPACKICNMM2015-48346, San Francisco, CA, July 6-10, 2015

[35]. K.E. Petersen, "Silicon as a mechanical material", Proceedings of the IEEE, Vol. 70, No. 5, 1982, pp. 420-457. 
[36]. S.R. Reddy, A. Abdoli, G.S. Dulikravich, C.C. Pacheco, G. Vasquez, R. Jha, M.J. Colaco and H.R.B. Orlande,"Multi-Objective Optimization of Micro Pin-Fin Arrays for Cooling of High Heat Flux Electronics with a Hot Spot," Paper InterPACKICNMM201548242, San Francisco, CA, July 6-10, 2015

[37]. S.R. Reddy, A. Abdoli, G.S. Dulikravich and R. Jha, "Conjugate Analysis of Thin Film Heat Spreaders to Reduce Temperature at Hot Spots,” ASME IMECE, Houston, TX, November 13-19, 2015

[38]. A.A. Balandin, "Thermal Properties of Graphene and Nanostructured Carbon Materials,” Nature Materials, Vol. 10, 2011, pp. 569-581 